\title{
Heavy-ion fusion reactions at extreme sub-barrier energies
}

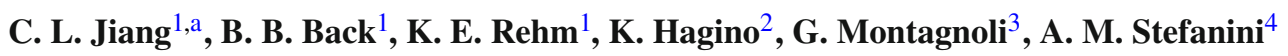 \\ ${ }_{1}^{1}$ Physics Division, Argonne National Laboratory, 9700 S. Cass Avenue, Argonne, IL 60439, USA \\ ${ }^{2}$ Department of Physics, Kyoto University, Kyoto 606-8502, Japan \\ ${ }^{3}$ Dipartimento di Fisica e Astronomia, Università di Padova, and INFN, Sez. di Padova, 35131 Padua, Italy \\ ${ }^{4}$ INFN, Laboratori Nazionali di Legnaro, 35020 Legnaro, Padova, Italy
}

Received: 26 May 2021 / Accepted: 13 June 2021 / Published online: 15 July 2021

(C) The Author(s) 2021

Communicated by Nicolas Alamanos

\begin{abstract}
The study of fusion reactions at extreme subbarrier energies has seen an increased interest in recent years, although difficult to measure due to their very small cross sections. Such reactions are extremely important for our understanding of the production of heavy elements in various environments. In this article, the status of the field is reviewed covering the experimental techniques, the available data, and the theoretical approaches used to describe such reactions. The fusion hindrance effect, first discovered in medium-mass systems, has been found to be relevant also for lighter systems. In some light systems, resonance structures are found to be important, while for heavy systems, the fission process plays an important role. In the near barrier region, couplings to collective excitations in the fusion participants and transfer reactions have been found to give a good description of the measured fusion cross sections and it results in a distribution of fusion barrier heights. New physics ingredients, related to the overlap process of the two projectiles, have to be introduced to describe the hindrance behavior. In addition, it has recently been found that the fusion cross section in both near-barrier and sub-barrier regions can be described very well in many cases using simple, analytical forms of the barrier-height distributions or a modified version of the classic Wong formula.
\end{abstract}

\section{Introduction}

Heavy-ion fusion, the most complex process in the interaction between two atomic nuclei has been studied extensively for more than 60 years, especially after the discovery of the sub-barrier fusion enhancement caused by couplings to intrinsic excitations of the two reaction partners. More than one thousand excitation functions have already been mea-

\footnotetext{
a e-mail: cjiang@anl.gov (corresponding author)
}

sured [1] to study the interplay between nuclear structure and dynamics in fusion reactions.

Heavy-ion fusion reactions are essential for efforts to extend the nuclear chart and for the synthesis of very heavy elements. In addition, some heavy-ion fusion reactions in lighter systems are central for our understanding of the reaction networks that support the energy production and elemental synthesis in stellar environments.

Fusion cross sections have been measured over a wide range of twelve orders of magnitude, from barn to $\mathrm{pb}$, much broader than for any other nuclear reaction (see recent reviews: [2-6]). In the early days of heavy-ion fusion studies, excitation functions in the range of hundreds of $\mathrm{mb}$ to $10 \mathrm{mb}$ have been measured and the Coulomb barrier height and a barrier radius were the first two parameters to be determined for various colliding systems. Deviations between the measured cross sections and the predictions by the classical formula observed at energies around the Coulomb barrier, sometimes called the sub-barrier fusion enhancement, led to the Coupled-Channels (CC) description [7,8]. For these studies, measurements in the cross section range down to $0.1 \mathrm{mb}$ were required. Subsequently, technologies were developed to perform precise, fine-energy-step measurements, which pushed the study to a new level in fusion dynamics by inspecting the barrier-height distributions $[9,10]$.

Again, deviations between measured cross sections and the CC calculations which appeared at the lowest measured energies for some colliding systems led the authors of Ref. $[11,12]$ to push the cross section measurements to the sub- $\mu \mathrm{b}$ region leading to the observation of a sharp drop-off in fusion cross section at the lowest energies which was identified as heavy-ion fusion hindrance at extreme sub-barrier energies [11-13].

The characteristics of fusion enhancement and fusion hindrance are illustrated in Fig. 1 for the system ${ }^{64} \mathrm{Ni}+{ }^{64} \mathrm{Ni}$. The curves are $\mathrm{CC}$ calculations (red solid) and potential model 


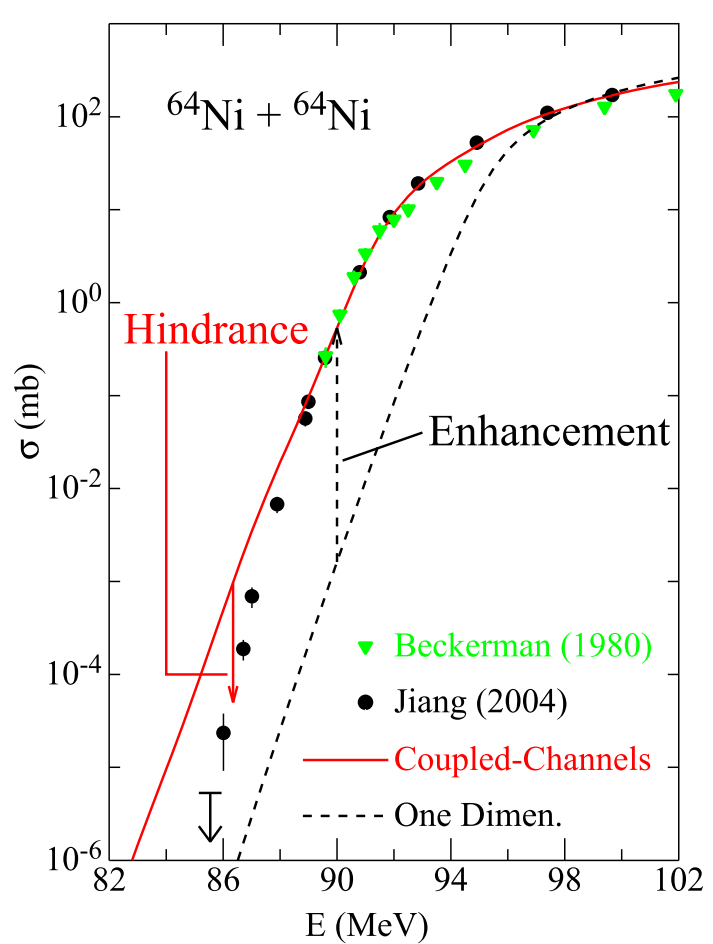

Fig. 1 Fusion excitation function for the system ${ }^{64} \mathrm{Ni}+{ }^{64} \mathrm{Ni}$ (data: Beckerman [16], Jiang [12]). The effects of fusion hindrance and fusion enhancement are illustrated. The fusion barrier is in this case about 97 $\mathrm{MeV}$

calculations without couplings (black dashed). The difference between the experimental data and the black curve displays the fusion enhancement, while the difference between the red curve and the measurements demonstrates the fusion hindrance at extreme subbarrier energies.

In the past, fusion reactions in light-mass systems at deep sub-barrier energies [14,15], which are of astrophysical interest, were sometimes considered as a different field. The observation of fusion hindrance, first discovered in heavier systems, can serve as a link between these two sub-fields.

The goal of the present article is to review the status of heavy-ion fusion studies at extreme sub-barrier energies, with a focus on the fusion hindrance phenomenon. Many experimental data have been published since the discovery of the hindrance effect in 2002. These data will be discussed and analyzed in conjunction with a reanalysis of previous experimental data.

\subsection{Structure of the article}

During the last 25 years, several review papers on the general subject of heavy-ion fusion reactions have been published [26]. Three of them also cover studies of the heavy-ion fusion hindrance at extreme sub-barrier energies [4-6]. Some new measurements of medium- and light-mass systems were subsequently published.
Recently, several empirical recipes for reproducing heavyion fusion excitation functions have been proposed (Refs. $[17,18])$, which can describe the fusion hindrance over a large mass range.

In addition to these reviews, the field of heavy-ion fusion reactions has been the subject of a series of international conferences [19-25].

This review is organized in the following way. After the Introduction, Sect. 2 provides a brief summary of the development of experimental techniques for fusion measurements that are mandatory for reaching the small cross section levels at which the hindrance effect appears.

The present theoretical understanding of the heavy-ion fusion theory, including the sub-barrier hindrance phenomenon, is discussed in Sect. 3.

Sections 4 and 5 contain the main presentation and discussion of the results pertaining to heavy-ion fusion hindrance, with heavy- and light-mass systems treated separately.

In Sect. 6, the systematics of the fusion hindrance phenomenon are presented, covering the whole mass region.

Section 7 introduces two new empirical recipes, which have not yet been widely discussed in the fusion community, but which can reproduce all the experimental data including those at extreme sub-barrier energies.

The final Sect. 8 provides the summary of the work and an outlook.

In this paper, the energy $E$ is the center-of-mass energy unless specified otherwise.

\subsection{Representations of the excitation function}

In most heavy-ion fusion experiments, the basic quantity measured is the total fusion cross section as a function of the collision energy, the so-called excitation function. It is often plotted directly as a function of energy either in linear or logarithmic scales. However, because of the steepness of excitation function at near- or sub-barrier energies, it may be difficult to recognize possible structures as well as deviations from theoretical curves, especially in logarithmic plots. In order to alleviate this problem, one often uses other representations in the comparison of measurements and theoretical calculations. A particular representation may emphasize the behavior of the excitation function in some part of the energy range. Of course, any theoretical calculation, which can reproduce the experimental excitation function must also reproduce all the other representations as well.

The fusion cross section for spin zero reaction partners is given by

$\sigma_{f u s}(E)=\frac{\pi}{k^{2}} \sum_{l}(2 l+1) T_{l}(E)$,

where $k$ is the wave number, $l$ is the orbital angular momentum, $T_{l}$ is the transmission coefficient for the orbital angu- 
lar momentum $l$, and $E$ is the center-of-mass energy in the collision. The simplest method for obtaining the $T_{l}$ needed for this calculation is the classical (black body) assumption where $T_{l}$ is assumed to be unity for $l$-values up to a sharp cut-off value of $l_{\max }$, and zero above this value. Here, $l_{\max }$ is the angular momentum for which the collision energy equals the Coulomb barrier, $V$, plus the centrifugal energy, $E_{\text {rot }}$. This approach leads to the expression for $\sigma_{c}(E) E$ [26],

$\sigma_{c}(E) E=\pi R^{2}(E-V)$,

where $R$ is the barrier radius. A more sophisticated analytical expression developed by Wong [27]: which includes the effects of quantum mechanical tunneling through the Coulomb barrier approximated by an inverted parabola potential, is given by

$\sigma_{w}(E) E=\frac{R^{2}}{2} \hbar \omega \ln [1+\exp ((2 \pi / \hbar \omega)(E-V))]$.

Here, $\omega$ is the frequency of the inverted harmonic potential. As expected, the Wong formula approaches the value given by Eq. (1) at energies above the Coulomb barrier where $\sigma(E) E$ vs $E$ is nearly a straight line (see Ref. [28]). For this reason, this representation of the data, $\sigma(E) E$, is frequently used since it allows for a simple derivation of the fusion barrier height and radius by simple linear fits to the asymptotic, above-barrier part of the excitation function.

Moreover, the first derivative

$d(\sigma(E) E) / d E$,

evaluated at well-above barrier energies, gives directly the value of $\pi R^{2}$ from which the barrier radius can be obtained.

In addition, within the coupled channel model, the second derivative of the quantity $\sigma(E) E$,

$d^{2}(\sigma(E) E) / d E^{2}$

represents, under certain simplifying assumptions, the distribution of fusion barrier heights, provided that the data are of sufficient quality (the data have to be measured in small enough energy steps) to allow for accurate estimates of the second derivative $[2,10]$.

At low energies relevant for astrophysical environments, the astrophysical $S$ factor $[13,29]$ and the logarithmic derivatives $[11,13]$ are often used to represent the experimental data. They are given by:

$S(E)=\sigma(E) E \exp (2 \pi \eta)$

and

$L(E)=d[\ln (\sigma(E) E)] / d E$.

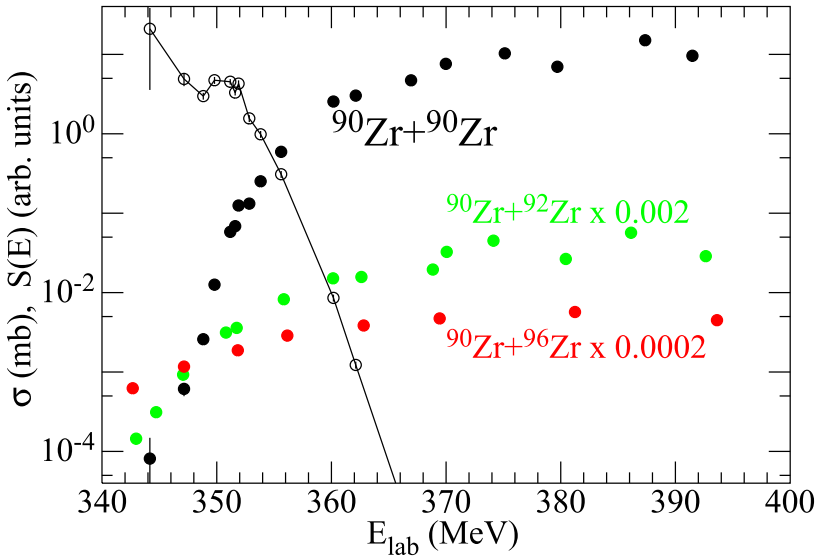

Fig. 2 Fusion excitation function of the system ${ }^{90} \mathrm{Zr}+{ }^{90} \mathrm{Zr}$ as a function of laboratory energy (black solid symbols). The green and red symbols are for the systems ${ }^{90} \mathrm{Zr}+{ }^{92} \mathrm{Zr}$ and ${ }^{90} \mathrm{Zr}+{ }^{96} \mathrm{Zr}$, scaled down by factors $2 \times 10^{-3}$ and $2 \times 10^{-4}$, respectively. The black open symbols and the solid curve are the $S(E)$ factor of ${ }^{90} \mathrm{Zr}+{ }^{90} \mathrm{Zr}$

Here $\eta$ is the Sommerfeld parameter,

$\eta=Z_{1} Z_{2} e^{2} /(\hbar v)$,

where $v$ is the relative velocity of the two heavy ions, and $Z_{1}, Z_{2}$ are their respective atomic numbers. The parameter $\eta$ is a quantity that determines the importance of the Coulomb effect [26].

Both of these representations will be used in the present review article. We emphasize that in discussing the physics behind a specific representation of the cross section, certain approximations are often involved. For example, referring to the second derivative $\frac{d^{2}(\sigma(E) E)}{d E^{2}}$ as a barrier-height distribution is done under the assumption of strong absorption. The observed value of $\frac{d^{2}(\sigma(E) E)}{d E^{2}}$ includes not only the distribution of fusion barrier heights, but also the effects of quantum mechanical tunneling through the barriers.

Similarly, one may relate the first derivative of $\sigma E$ to the transmission coefficient, $T(E)$,

$\frac{d(E \sigma)}{d E}=\pi R^{2} T(E)$.

This representation is often used at energies above the Coulomb barrier, and assumes the strong absorption approximation [30].

During the 1980's, measurements of spin distributions of compound nuclei formed via heavy-ion fusion were obtained, and explained by an $l$-dependent representation, $d \sigma_{l} / d l$ [31]. The very small cross sections at extreme sub-barrier energies have so far prevented the use of this representation, although some predictions about the $d \sigma_{l} / d l$ have been obtained from the CC calculations for the explanation of fusion hindrance in different models. 


\section{Experimental techniques}

\subsection{Experimental challenges}

For such sub-barrier measurements, detectors with high efficiency, high-intensity beams with well known energies and targets, which can handle these beams, are needed. Special effort has to be placed on understanding possible background effects, especially in the range of cross section below $10 \mu \mathrm{b}$.

Non-monoisotopic targets present a special challenge, in particular if the interest is focused on the lightest isotope. For example, bombarding a ${ }^{58} \mathrm{Ni}$ target with a ${ }^{58} \mathrm{Ni}$ beam will have contributions from reactions of the ${ }^{64} \mathrm{Ni}$ contaminant in the target with the ${ }^{58} \mathrm{Ni}$ beam. Because of the lab to c.m. conversion, the c.m. energy for the ${ }^{64} \mathrm{Ni}$ target contaminant is considerably higher, which affects the ${ }^{58} \mathrm{Ni}+{ }^{58} \mathrm{Ni}$ measurement at the lowest energies. At energies $E_{l a b}<185$ $\mathrm{MeV}$, the fusion yields will be dominated by the ${ }^{64} \mathrm{Ni}$ contaminant even at the low abundance values of $2 \times 10^{-4}$. For that reason the cross section measurement for fusion of ${ }^{58} \mathrm{Ni}+{ }^{58} \mathrm{Ni}$ in Ref. [16,32] has not yet been extended to lower energies.

In Fig. 2, we present an analysis for a measurement of fusion in the system of ${ }^{90} \mathrm{Zr}+{ }^{90} \mathrm{Zr}$ [33]. The excitation function of ${ }^{90} \mathrm{Zr}+{ }^{90} \mathrm{Zr}$ is shown by black solid symbols plotted as a function of the laboratory energy. Also shown by red and green solid symbols are the excitation functions of ${ }^{90} \mathrm{Zr}+{ }^{96} \mathrm{Zr}$ and ${ }^{90} \mathrm{Zr}+{ }^{92} \mathrm{Zr}$, but scaled down by factors of $2 \times 10^{-3}$ and $2 \times 10^{-4}$, respectively, which are typical values of these target contaminations. As can be seen, at energies below $\approx 350 \mathrm{MeV}$, small target contaminations of ${ }^{92} \mathrm{Zr}$ and ${ }^{96} \mathrm{Zr}$ may dominate the measured excitation function. The resulting $S$ factor of ${ }^{90} \mathrm{Zr}+{ }^{90} \mathrm{Zr}$, shown by black open symbols and the solid curve, exhibits a maximum followed by a continuous rise, originating from the target contamination of heavier isotopes. In fact, the excitation functions measured for ${ }^{90} \mathrm{Zr}+{ }^{96} \mathrm{Zr}$ and ${ }^{90} \mathrm{Zr}+{ }^{92} \mathrm{Zr}$ show clear $S$ factor maxima without the additional increase towards lower energies, (see Fig. 29 below for ${ }^{90} \mathrm{Zr}+{ }^{92} \mathrm{Zr}$.) Thus, in order to avoid this difficulty in fusion measurements at extreme sub-barrier energies, monoisotopic targets or the heaviest isotopes of a given element should be employed in the experiments. Otherwise, targets with extremely high isotopic enrichment must be used.

In addition to problems originating from target contaminants, some experiments also suffer from impurities of the ion beam. Electron Cyclotron Resonance (ECR) ion sources are known to have a so-called 'memory effect' [35] from samples used in previous experiments. The effect of such a contamination is shown in Fig. 3 from an experiment studying fusion reactions between a ${ }^{58} \mathrm{Ni}$ beam and monoisotopic ${ }^{89} \mathrm{Y}$ at the ATLAS accelerator [36]. At a laboratory energy of

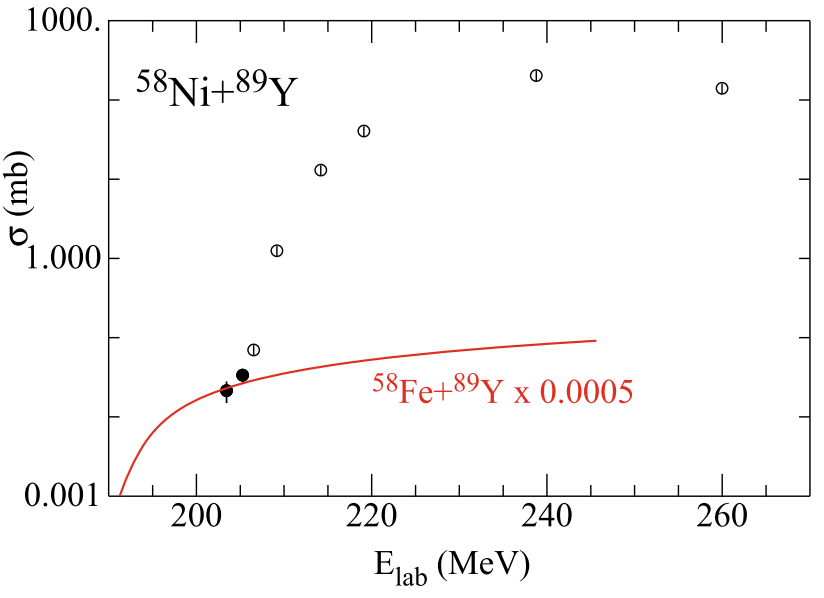

Fig. 3 Effect of a small ${ }^{58} \mathrm{Fe}$ beam contamination from a measurement of fusion cross sections in the system ${ }^{58} \mathrm{Ni}+{ }^{89} \mathrm{Y}$ at the ATLAS accelerator plotted as a function of the laboratory energy. Since no experimental data for ${ }^{58} \mathrm{Fe}+{ }^{89} \mathrm{Y}$ exist, the high-energy part of the ${ }^{58} \mathrm{Ni}+{ }^{89} \mathrm{Y}$ excitation function has been shifted down by the difference in the respective Coulomb barriers as calculated with the Bass model [34] ( $\Delta V=11.28$ $\mathrm{MeV}$ ) and scaled down by a factor of 2000 (red solid) to reproduce the two lowest energy points

$\sim 205 \mathrm{MeV}$ the excitation function shows a slight change in slope which is not observed for the similar system ${ }^{60} \mathrm{Ni}+{ }^{89} \mathrm{Y}$ [11]. Since ${ }^{89} \mathrm{Y}$ is a monoisotopic element this cannot originate from the presence of a heavier target contaminant as for the case of ${ }^{58} \mathrm{Ni}+{ }^{58} \mathrm{Ni}$. However, ${ }^{58} \mathrm{Ni}$ has a lower $\mathrm{Z}$ isobar, ${ }^{58} \mathrm{Fe}$, for which the Coulomb barrier for ${ }^{58} \mathrm{Fe}+{ }^{89} \mathrm{Y}$ fusion is lower than for ${ }^{58} \mathrm{Ni}+{ }^{89} \mathrm{Y}$. Shifting the experimental ${ }^{58} \mathrm{Ni}+{ }^{89} \mathrm{Y}$ excitation function by the difference in the Coulomb barriers, calculated with the Bass model [34], $\left(V_{C}=137.33 \mathrm{MeV}\right.$ and $126.05 \mathrm{MeV}$ for projectile ${ }^{58} \mathrm{Ni}$ and ${ }^{58} \mathrm{Fe}$, respectively), and using a beam contamination ratio of about 0.0005 (i.e. $0.5 \mathrm{nA}$ in the beam intensity of $1 \mu \mathrm{A}$ used in the experiment) gives a good description of the experimental data.

While each fusion system encounters different background problems, the use of a beam particle which does not have a stable isobaric partner with lower $Z$ and the use of the heaviest isotope as a target can in most cases eliminate the experimental difficulties mentioned above.

In addition, chemical contaminations from ubiquitous elements can lead to additional backgrounds. An example is the presence of hydrogen (and deuterium) in carbon targets which, through the ${ }^{12} \mathrm{C}(\mathrm{d}, \mathrm{p})$ reaction, can lead to background protons which will interfere with protons from the ${ }^{12} \mathrm{C}\left({ }^{12} \mathrm{C}, \mathrm{p}\right){ }^{23} \mathrm{Na}$ fusion channel [37]. For this reason, highlyordered pyrolytic-graphite (HOPG) has been used in some of the experiments. This material is known to have very high purity, which minimizes the contribution from the ${ }^{12} \mathrm{C}(\mathrm{d}, \mathrm{p})$ background reaction mentioned above. 


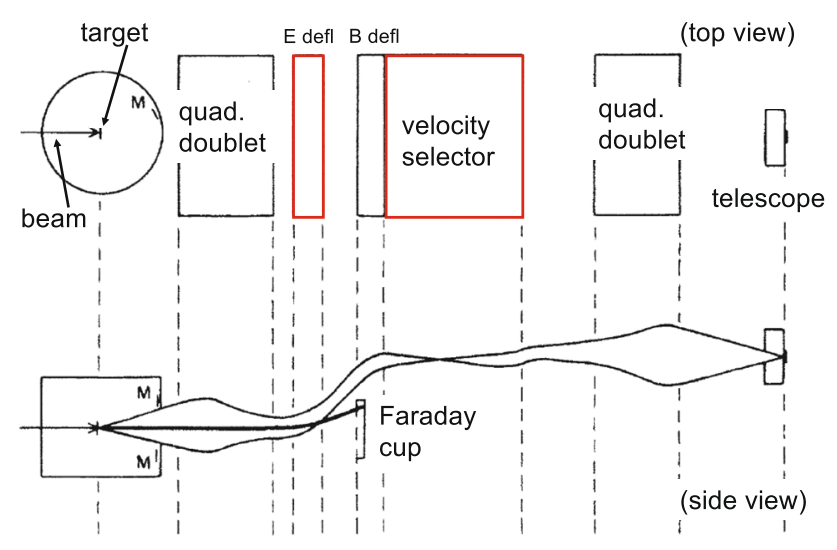

Fig. 4 Schematics of the MIT-BNL Recoil Mass Selector, composed of a magnetic quadrupole doublet, a small electrostatic deflector, a Wien filter and a final quadrupole doublet.The ERs were focused onto a detector telescope (see text). Figure modified from Ref. [16]

\subsection{Advanced facilities for low cross section measurements}

A variety of experimental set-ups have been utilized at facilities worldwide to study heavy-ion fusion reactions at very low energies starting from the 1980's. Below we present a short description of some devices that have been relevant for obtaining the results reported in the following sections.

The experimental set-up used in the pioneering experiments of Beckerman et al. [16,32] on fusion in the $\mathrm{Ni}+\mathrm{Ni}$ systems is schematically shown in Fig. 4. Direct detection of the evaporation residues (ERs) at $0^{\circ}$ and at small angles with respect to the beam was accomplished, following separation from the beam and beam-like ions by means of an electrostatic deflector and a $\mathrm{E} \times \mathrm{B}$ crossed-field velocity selector. A $\Delta$ E-E telescope detected and identified the ERs, by using a proportional counter filled with isobutane gas and a 450 $\mathrm{mm}^{2}$ silicon surface barrier detector mounted at the rear of the telescope.

Several experiments on low-energy fusion reactions, including the first one where the hindrance phenomenon was observed, were performed with the Fragment Mass Analyzer (FMA) [38] at the Argonne superconducting linear accelerator ATLAS. A layout of the FMA is shown in Fig. 5. It has large momentum and angular acceptances (10\% and $\theta_{l a b} \leq 2.9^{\circ}$, respectively), that allow a high detection efficiency for the ERs (50-70\% for each charge state), and beam suppression factor (about $4 \times 10^{17}$ ) [39]. In the following sections we will report on several measurements done using the FMA since 2001.

The velocity filter SHIP was installed at GSI, Germany, in the late 1970's. It consists (see Fig. 6) of a sequence of magnetic and electric fields with a very high beam rejection capability and a high efficiency for transporting the ERs to the focal plane detectors. The main purpose of SHIP was synthesizing superheavy elements using fusion reactions, and

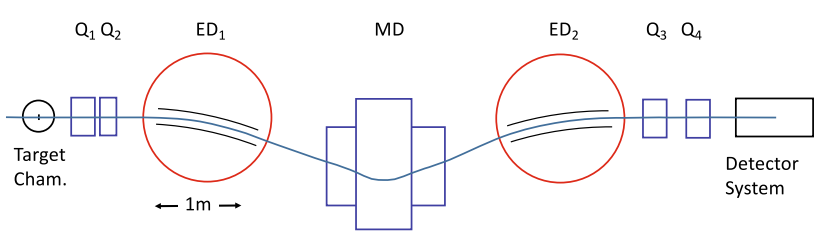

Fig. 5 Layout of the FMA at ATLAS. $\mathrm{ED}_{1}$ and $\mathrm{ED}_{2}$ are electric dipoles, and $\mathrm{MD}$ is a magnetic dipole. $\mathrm{Q}_{1}, \mathrm{Q}_{2}, \mathrm{Q}_{3}$ and $\mathrm{Q}_{4}$ are magnetic quadrupoles. The target chamber and the detector system are also indicated. Figure redrawn and modified from Ref. [39]

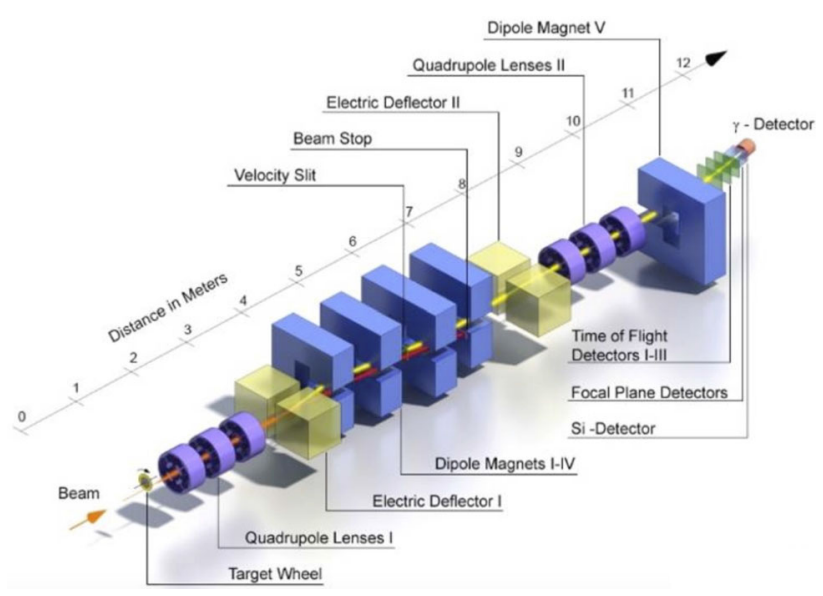

Fig. 6 The velocity filter SHIP, installed at GSI $[42,43]$

great success was achieved in this endeavor [40]. This topic is outside the scope of the present review. Several relevant studies were also performed on heavy-ion fusion below the barrier, e.g. the fusion measurements in the $\mathrm{Zr}+\mathrm{Zr}$ systems shown in Fig. 2, (see also Reisdorf [41]).

Several setups have been developed and constructed at the Australian National University (ANU), Canberra, for the measurement of low-energy fusion cross sections, such as the velocity filter separating the ERs from beam-like and elastic scattering ions at very small angles. The first experiment (and several others later on) deriving fusion barrier distributions from careful measurements of fusion excitation functions, were performed using this velocity filter [2,9]. The capabilities of the ANU setup has been a fundamental ingredient for the progress of fusion studies in the 1990's.

Fusion-fission fragments in coincidence $[44,45]$ are detected at ANU by the set-up named Cube. It is complementary to the velocity filter, and consists of large-area multiwire proportional counters MWPC, that are X, Y position sensitive. The center of the MWPC's is placed at $180 \mathrm{~mm}$ from the target, so that scattering angles of $95^{\circ} \leq \theta_{l a b} \leq 170^{\circ}$ in the backward hemisphere, and $10^{\circ} \leq \theta_{l a b} \leq 85^{\circ}$ in the forward hemisphere are covered. Energy loss and time of arrival are provided by the central foils of the two MWPC's. Only coincident signals between the two detectors are acquired. 


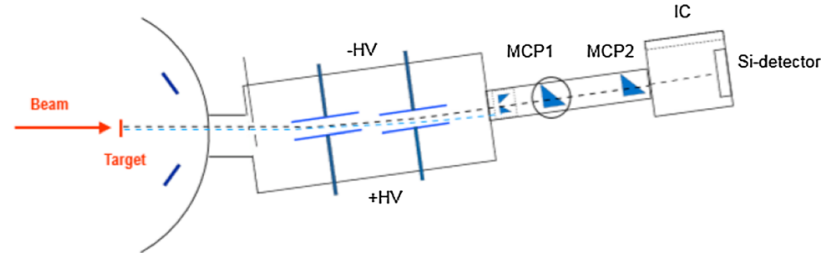

Fig. 7 The PISOLO set-up used for measurements of fusion cross sections at LNL. Figure partially redrawn from Ref. [46]

A gas-filled 6.5 T superconducting solenoid SOLITAIRE [47] was also developed and installed at ANU. The ERs are detected with very high efficiency because their angular distribution is covered in a single measurement from $0.45^{\circ}$ to $9.5^{\circ}$.

About 30 years ago, fusion cross sections covering four orders of magnitude were measured at Oak Ridge National Laboratory (ORNL) for the systems ${ }^{46,50} \mathrm{Ti}+{ }^{90} \mathrm{Zr},{ }^{93} \mathrm{Nb}$ [48], using a velocity filter. It consists of two electrostatic deflectors separated by a dipole magnet. A quadrupole doublet followed the deflectors to focus the ERs onto the focal plane detector consisting of a $\Delta \mathrm{E}-\mathrm{E}$ ionization chamber followed by a silicon detector. The whole set-up could rotate around the target to measure the angular distribution of ERs with a solid angle of $\approx 1 \mathrm{msr}$.

The two systems ${ }^{46,50} \mathrm{Ti}+{ }^{124} \mathrm{Sn}$ were investigated more recently [49]. The first part of these measurements were performed at the Holifield Radioactive Ion Beam Facility (HRIBF), using a ${ }^{124} \mathrm{Sn}$ beam on titanium targets in inverse kinematics. The ERs were identified in a system consisting of three micro-channel plate detectors and a TOF- $\Delta \mathrm{E}-\mathrm{E}$ telescope [50], providing the $Z$ identification. The second part of the experiment on ${ }^{46,50} \mathrm{Ti}+{ }^{124} \mathrm{Sn}$ followed at the Australian National University in direct kinematics with beams of ${ }^{46,50} \mathrm{Ti}$ using the superconducting solenoid SOLITAIRE.

Fusion-evaporation cross sections have been measured at Laboratori Nazionale di Legnaro (LNL) since 1983 using the set-up PISOLO, which is based on an electrostatic separator [51]. Its present layout is shown in Fig. 7. A two-stage electrostatic deflector follows an entrance collimator; most of the beam and beam-like particles are stopped on one side of the exit collimator which allows ER to enter the Energy-ToF$\Delta \mathrm{E}$ detector telescope consisting two micro-channel plate detectors, an ionization chamber and a silicon detector. The original configuration underwent various upgrades in recent years [46], that have allowed to measure cross sections in the extreme sub-barrier energy range, down to $0.5-1 \mu \mathrm{b}$.

In more recent years, the group at the China Institute of Atomic Energy of Beijing, China [52] installed a similar setup, that has allowed reliable measurements of fusion excitation functions for ${ }^{32} \mathrm{~S}+{ }^{90,96} \mathrm{Zr}$ [53] and for ${ }^{16} \mathrm{O}+{ }^{74,76} \mathrm{Ge}$ [54].

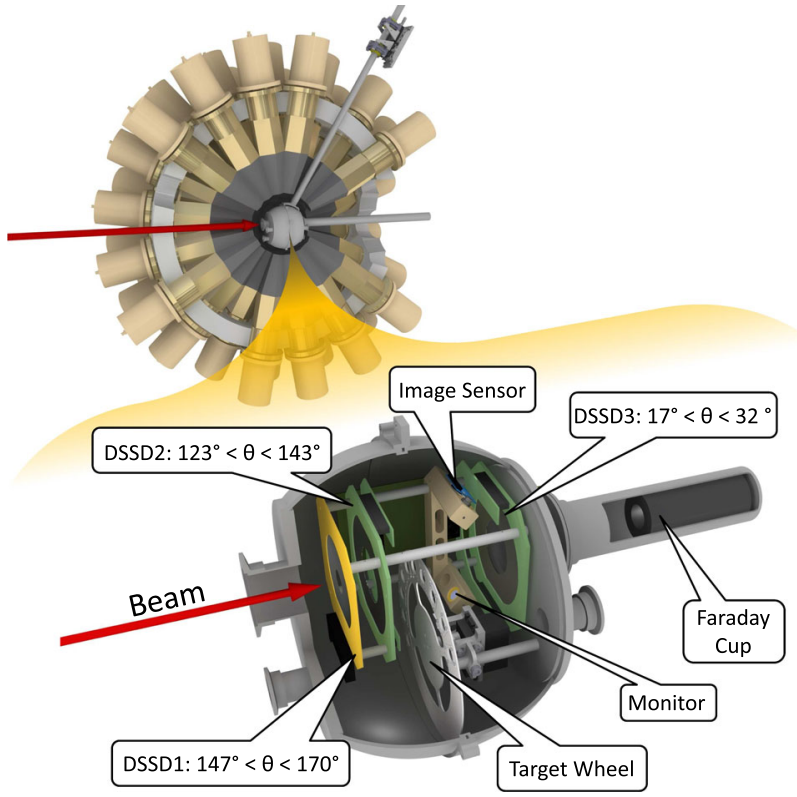

Fig. 8 Schematic of the experimental setup at ANL for the first particle- $\gamma$ coincidence measurement at astrophysical energies. The spherical target chamber is mounted in the middle of the Gammasphere array $[55,56]$

The Heavy-ion reaction analyzer, HIRA at the Inter University Accelerator Centre (IUAC), New Dehli, is a recoil mass spectrometer (RMS) having a high rejection factor for the primary beam $\approx 10^{13}$, that allows to operate it in the beam direction. Similar to the Fragment Mass Separator, FMA at ATLAS, the HIRA spectrometer [57] has a symmetric electrostatic dipole-magnetic dipole-electrostatic dipole (ED-MD-ED) configuration. Two quadrupole doublets are placed upstream and downstream of the two electric dipoles. A very good mass resolution $(\simeq 1 / 300)$ is obtained at the focal plane for the reaction products. HIRA offers also a variable acceptance 1-10 msr, and energy and mass acceptances $\pm 20 \%$ and $\pm 5 \%$, respectively. The sliding seal scattering chamber allows the device to rotate up to $25^{\circ}$. A $\gamma$-ray array can be installed around the target by using another small $\mathrm{Al}$ scattering chamber.

$\gamma$-ray spectroscopy [37] and particle-spectroscopy [58] have been used for measuring fusion of light-mass systems at astrophysical sub-barrier energies at the RuhrUniversität Bochum (Germany) and at the Center for Isotopic Research on the Cultural and Environmental heritage (CIRCE), Caserta (Italy) [59].

At Argonne (ANL) the Gammasphere array [60] was operated in a combined setup with silicon detectors placed near the target for the $\gamma$-particle-coincidence study of ${ }^{12} \mathrm{C}+{ }^{12} \mathrm{C}$ $[55,56]$. A schematic drawing of the setup is shown in Fig. 8. Cross sections down to $\approx 6 \mathrm{nb}$ were measured [56].

A different set-up was recently developed at the University of Notre Dame exploiting a 5 MV Pelletron accelerator. 


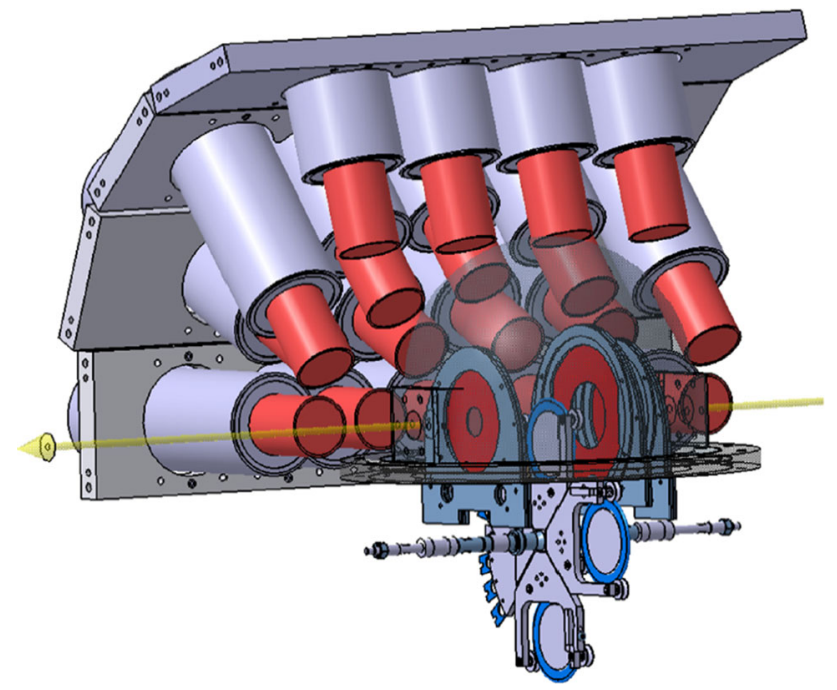

Fig. 9 The STELLA set-up includes important developments for reaching the picobarn cross section range. Three annular silicon detectors and a rotating target capable to sustain beam intensities above $10 \mu \mathrm{A}$, are installed. The direction of the beam (from right to left) is given by the yellow arrow. Only half of the $\mathrm{LaBr}_{3}(\mathrm{Ce}) \gamma$-ray is shown. See text for details

As significant results, we mention the fusion measurements of ${ }^{12} \mathrm{C}+{ }^{16} \mathrm{O}[61]$ and ${ }^{12} \mathrm{C}+{ }^{12} \mathrm{C}$ [62]. High-intensity oxygen and carbon beams impinged on thick, ultrapure graphite targets. Protons and $\gamma$-rays were simultaneously measured for singles and for coincidence events, using an array of silicon detectors, and an HpGe detector close to the thick target.

Another experimental set-up called STELLA, designed and constructed in Strasbourg [63], is optimized for the measurement of extreme sub-barrier light heavy-ion fusion cross sections. It is installed at the Andromède accelerator of the Institut de Physique Nucléaire, Orsay (France) and it is able to determine very small cross sections in the picobarn range. STELLA is based on the coincident measurement of emitted $\gamma$-rays using FATIMA [64] (an array of $\mathrm{LaBr} 3(\mathrm{Ce}$ ) scintillators), and of evaporated charged particles using annular silicon detectors. A rotating target was employed in this setup. It has been used for a study of the ${ }^{12} \mathrm{C}+{ }^{12} \mathrm{C}$ fusion reaction [65] and is shown in Fig. 9.

A measurement of the ${ }^{12} \mathrm{C}+{ }^{13} \mathrm{C}$ fusion reaction was performed by detecting the decay of the residual nucleus ${ }^{24} \mathrm{Na}$ with a half-life of $\mathrm{T}_{1 / 2}=15 \mathrm{~h}$, following proton evaporation. The 3 MV Tandetron of the Horia Hulubei National Institute for R\&D in Physics and Nuclear Engineering (IFIN$\mathrm{HH})$ provided a ${ }^{13} \mathrm{C}$ beam with a current up to $15 p \mu \mathrm{A}$ on a $1.5 \mathrm{~mm}$ thick natural carbon target [66]. At low energies the irradiated targets were transported (in 3 hours) to the underground laboratory in the SLANIC salt mine (Romania) for $\gamma$-ray measurements [67]. Cross sections down to the $10 \mathrm{pb}$ range were determined [68].
Recently, studies of fusion hindrance in asymmetric systems with light projectiles $\left({ }^{6,7} \mathrm{Li},{ }^{12} \mathrm{C}\right)$ on the heavy target ${ }^{198} \mathrm{Pt}$, were performed by Shrivastava et al. [69,70]. Fusion cross sections were measured down to $\approx 100 \mathrm{nb}$ using an offline technique where coincidences between characteristic Xand $\gamma$-rays were detected [71].

The Argonne Gas-Filled Fragment Analyzer, AGFA is a new gas-filled separator [72], recently developed and installed at ANL. It is based on an innovative quadrupoledipole design and has an overall length $\simeq 4 \mathrm{~m}$, with the following features: (1) high efficiency (up to $\approx 70 \%$ ) for ER detection, (2) small image size at the focal plane, where a large double-sided Si strip detector is mounted, (3) a maximum $\mathrm{B} \rho$ of $2.5 \mathrm{Tm}$ (bending angle $38^{\circ}$ ) and (4) the ability to work in a combined set-up with Gammasphere and/or with a gas catcher for the production of exotic beams of radioactive ions. The solid angle of AGFA exceeds $40 \mathrm{msr}$ in stand-alone mode. AGFA has not yet been used for experiment of fusion excitation functions.

The hybrid recoil mass analyzer (HYRA) [73] is installed at the IUAC in New Delhi with a dual mode/dual stage configuration. The layout of the first stage is Q1Q2-MD1-Q3-MD2Q4Q5 (Q are the magnetic quadrupoles and MD the magnetic dipoles), operating with momentum dispersion in gas-filled mode or as a momentum achromat in vacuum mode. The second stage is Q6Q7-ED-MD3-Q8Q9, thus producing a mass dispersion at the focal plane.

Several experiments were performed in recent years using the first stage of HYRA in gas-filled mode. Among those experiments we cite the spin distributions and ER cross sections in ${ }^{28} \mathrm{Si}+{ }^{176} \mathrm{Yb}$ by Sudarshan et al. [74], and fusionevaporation in studies of the ${ }^{16} \mathrm{O}+{ }^{194} \mathrm{Pt}[75],{ }^{19} \mathrm{~F}+{ }^{194,196,198}$ $\mathrm{Pt}[76]$ and ${ }^{31} \mathrm{P}+{ }^{170} \mathrm{Er}$ [77] reactions.

\subsection{Indirect methods}

One of the big experimental challenges for measurements at deep subbarrier energies originates from the extremely small fusion cross sections. To overcome this problem, several indirect methods have been conceived [78]. Among them, the Trojan horse method, THM, has been successfully applied to many nuclear astrophysical reactions. The basic idea of this method is schematically shown in Fig. 10. Here, the projectile consists of two parts $(a=X+s)$ and one of them, $X$, is transferred to the target nucleus, $A$, to form a compound nucleus. The other fragment, $s$, acts as a spectator. Even when the relative energy between the fragment $X$ and the target $A$ may be low, the incident energy of the projectile $a$ can be set to a much higher energy. In this way, the Coulomb barrier is easily overcome, and at the same time, the electron screening effect can become negligibly small.

In order to relate the three-body reaction of $(X+s)+A$ to the virtual two-body reaction of $X+A$, theoretical cal- 


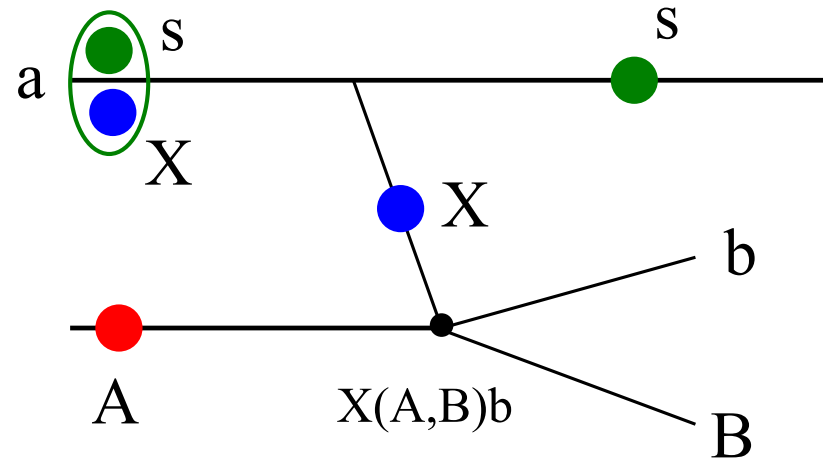

Fig. 10 A schematic description of the Trojan horse method. The projectile nucleus $a$ consists of two fragments, $X$ and $s$, for which the fragments $X$ fuses with the target nucleus $A$ while the fragment $s$ acts as a spectator. After $X$ fuses with $A$, the compound nucleus decays into $B+b$

culations have to be applied, where the plane-wave impulse approximation has often been used [79].

Recently, the Trojan horse method was applied to the astrophysically important ${ }^{12} \mathrm{C}+{ }^{12} \mathrm{C}$ fusion reaction [80]. The validity of the results has been called into question by theoretical calculations, which will be discussed further in Sect. 5.

\section{Theoretical models}

\subsection{Potential model}

The simplest approach to nuclear fusion reactions is to employ a potential model, in which the projectile and the target nuclei are assumed to be inert particles. Vaz, Alexander, and Satchler [81], as well as Bass [82], employed this idea and empirically determined fusion barriers in a systematic way. Once a potential is given, one can use a simple formula, Eq. (3), derived by Wong [27] to estimate fusion cross sections. Even though the potential model does not work for heavy systems at energies below the Coulomb barrier, as we will discuss in Sect. 3.3, it still provides useful reference cross sections to discuss dynamical effects in heavy-ion fusion reactions.

In the potential model, the interaction between a projectile and a target nucleus is modeled by a spherical complex potential,

$V(r)=V_{N}(r)+V_{C}(r)-i W(r)$,

where $r$ is the distance between centers of the colliding nuclei, $V_{N}(r)$ and $V_{C}(r)$ are the nuclear and the Coulomb parts of the potential, respectively. The imaginary part of the potential, $-i W(r)$, simulates a compound nucleus formation as an absorption of the incident flux inside the Coulomb barrier.
The radial Schrödinger equation is solved with the regular boundary condition at the origin, together with the asymptotic boundary condition given by

$u_{l}(r) \rightarrow H_{l}^{(-)}(k r)-S_{l} H_{l}^{(+)}(k r) \quad(r \rightarrow \infty)$,

where $u_{l}(r)$ is the radial wave function for the partial wave $l$, and $S_{l}$ is the $S$-matrix. $H_{l}^{(-)}(k r)$ and $H_{l}^{(+)}(k r)$ are the incoming and the outgoing Coulomb wave functions, respectively, and $k$ is the wave number, given as $k=\sqrt{2 \mu E / \hbar^{2}}, \mu$ and $E$ being the reduced mass and the incident energy in the center of mass frame, respectively. The fusion cross sections are then obtained from Eq. 1 with $T_{l}=1-\left|S_{l}\right|^{2}$

$\sigma_{\text {fus }}(E)=\frac{\pi}{k^{2}} \sum_{l}(2 l+1)\left(1-\left|S_{l}\right|^{2}\right)$.

Notice that with the boundary condition of Eq. (11) the factor $1-\left|S_{l}\right|^{2}$, can also be expressed as [83],

$1-\left|S_{l}\right|^{2}=\frac{2 \mu}{k \hbar^{2}} \int_{0}^{\infty} d r W(r)|u(r)|^{2}$.

The regular boundary condition at the origin is often replaced by the incoming wave boundary condition (IWBC) given by $[4,84-86]$

$u_{l}(r)=\sqrt{\frac{k}{k_{l}(r)}} \tilde{T}_{l} \exp \left(-i \int_{r_{\mathrm{abs}}}^{r} k_{l}\left(r^{\prime}\right) d r^{\prime}\right) \quad\left(r \leq r_{\mathrm{abs}}\right)$,

where

$k_{l}(r)=\sqrt{\frac{2 \mu}{\hbar^{2}}\left(E-V_{N}(r)-V_{C}(r)-\frac{l(l+1) \hbar^{2}}{2 \mu r^{2}}\right)}$

is the local wave number, which takes into account the real part of the potential $V(r)$ and the centrifugal potential, and $r_{\mathrm{abs}}$ is the absorption radius. $\tilde{T}_{l}$ is the transmission coefficient. The main assumption here is that the absorption is so strong that the incoming flux never bounces back. This is achieved by taking $W(r)$ large enough in Eq. (10) and at the same time neglecting the reflected flux due to $-i W(r)$. In the IWBC model, one does not need to introduce explicitly the imaginary part of the internuclear potential. Moreover, the penetration probability, $1-\left|S_{l}\right|^{2}$, in Eq. (12) can be replaced by $1-\left|S_{l}\right|^{2}=\left|T_{l}\right|^{2}$, which has a large numerical advantage at energies well below the Coulomb barrier [4].

Figure 11 shows as an example the astrophysical $S$ factor (see Eq. (6)) obtained by the potential model calculations for the ${ }^{16} \mathrm{O}+{ }^{16} \mathrm{O}$ reaction. The solid line employs a WoodsSaxon potential with the depth parameter of $V_{0}=-54.5$ $\mathrm{MeV}$, a radius of $R=6.5 \mathrm{fm}$, and a diffuseness parameter of $a=0.45 \mathrm{fm}$. One can see that this calculation reproduces the experimental data for this system quite well. In the literature, a simple square-well potential has also been used for a nuclear potential [88-93]. The dashed curve in Fig. 11 was obtained 


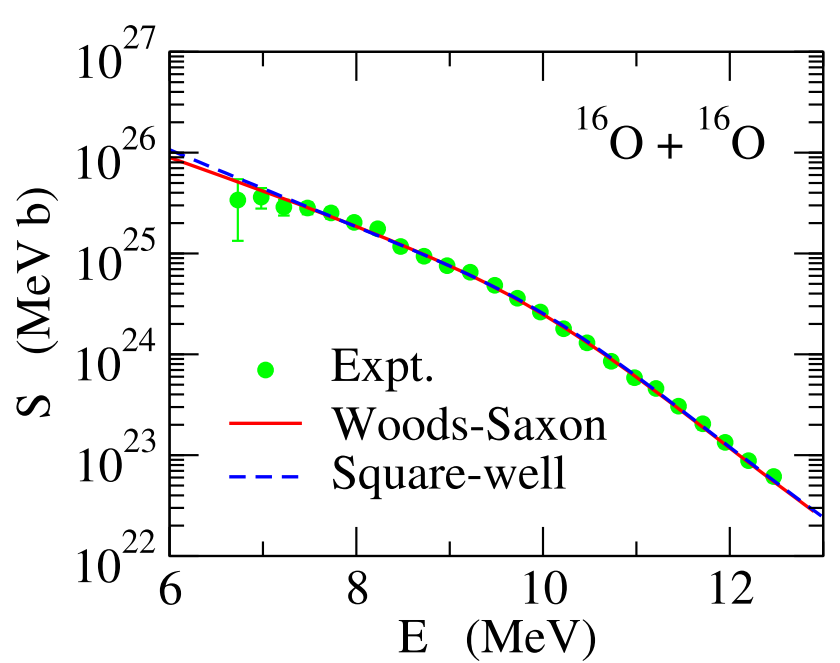

Fig. 11 The astrophysical $S$-factor for the ${ }^{16} \mathrm{O}+{ }^{16} \mathrm{O}$ reaction obtained with the potential model. The solid and the dashed lines show results of a Woods-Saxon potential and a square-well potential, respectively. The data are taken from Ref. [87]

with the depth and the radius of the square-well potential of $V_{0}=-9.4 \mathrm{MeV}$ and $R=8.13 \mathrm{fm}$. Even though this potential is significantly shallower and wider than the WoodsSaxon potential [93], it reproduces the data equally well.

Figure 12 compares similar potential model calculations for a light, ${ }^{14} \mathrm{~N}+{ }^{12} \mathrm{C}$, and a heavy, ${ }^{16} \mathrm{O}+{ }^{154} \mathrm{Sm}$, system. For the ${ }^{14} \mathrm{~N}+{ }^{12} \mathrm{C}$ system, the potential model works remarkably well as for the ${ }^{16} \mathrm{O}+{ }^{16} \mathrm{O}$ system shown in Fig. 11. In contrast, for the heavier system, ${ }^{16} \mathrm{O}+{ }^{154} \mathrm{Sm}$, it considerably underestimates the fusion cross sections at energies below the Coulomb barrier, which is about $59 \mathrm{MeV}$ for this system. Interestingly, the potential model still works at energies above the Coulomb barrier. These features can be understood in terms of the static deformation of the target nucleus, ${ }^{154} \mathrm{Sm}$ [4] (see Sect. 3.3).

Besides the Woods-Saxon and the square-well potentials, a double-folding potential [96] with an effective nucleonnucleon interaction, such as the density-dependent Michigan three-range Yukawa (DDM3Y) interaction [97,98], as well as the Sao Paulo potential [99], have also been employed for an internuclear potential. In the double folding model, the internuclear potential is constructed as

$V_{N}(r)=\int d \boldsymbol{r}_{1} d \boldsymbol{r}_{2} \rho_{1}\left(\boldsymbol{r}_{1}\right) \rho_{2}\left(\boldsymbol{r}_{2}\right) v_{\mathrm{NN}}\left(\boldsymbol{r}+\boldsymbol{r}_{2}-\boldsymbol{r}_{1}\right)$,

where $\rho_{i}(\boldsymbol{r})$ is the density distribution of the nucleus $i$ and $v_{\mathrm{NN}}(\boldsymbol{r})$ is an effective nucleon-nucleon interaction. The Sao Paulo potential is in fact based also on the double folding procedure and takes into account the effect of non-locality of the potential as a velocity dependence. Recently, a potential based on the time-dependent Hartree-Fock (TDHF) method has also been developed. In this approach, the potential is constructed using the density-constrained TDHF method
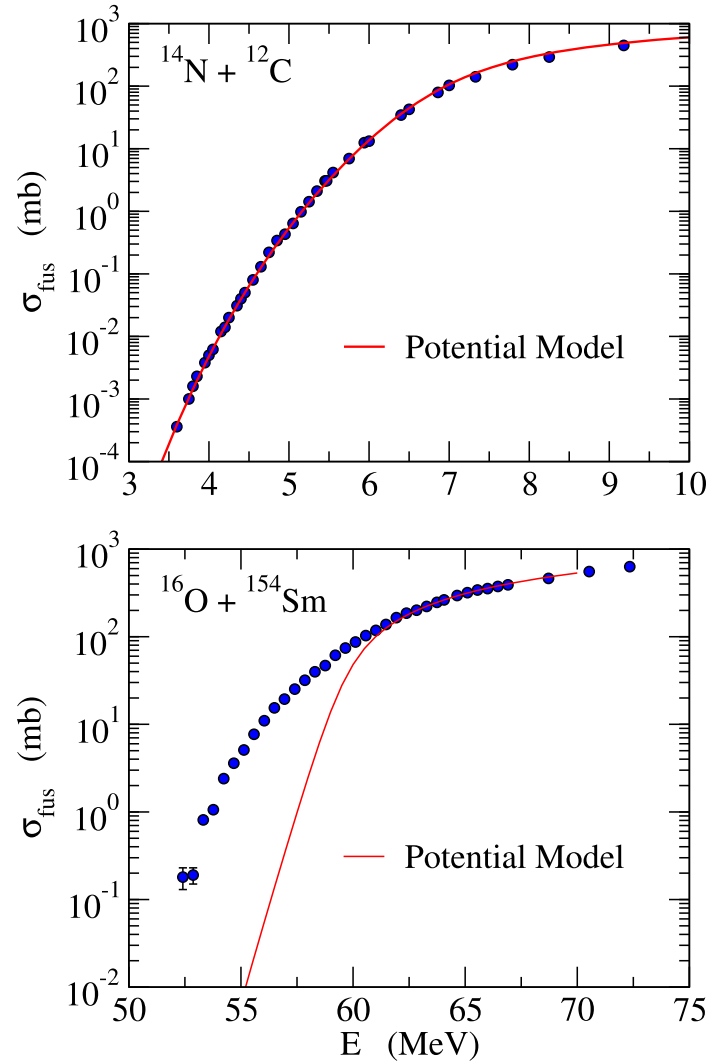

Fig. 12 Potential model calculations for the ${ }^{14} \mathrm{~N}+{ }^{12} \mathrm{C}$ system (upper panel) and for the ${ }^{16} \mathrm{O}+{ }^{154} \mathrm{Sm}$ system (lower panel). The experimental data are taken from Refs. [94,95]

(DC-TDHF) $[100,101]$, in which the total energy is minimized at each internuclear distance under the condition that the density distribution of the system coincides with that obtained with a TDHF time evolution. Even though the TDHF method itself cannot describe a many-body tunneling phenomenon, and thus cannot be applied to fusion at energies below the Coulomb barrier, the DC-TDHF method can still be used to estimate fusion cross sections in that energy region. Recently, this method has successfully been applied to the ${ }^{12} \mathrm{C}+{ }^{12} \mathrm{C}$ system, suggesting that the astrophysical $S$-factor for this system does not drop off significantly even at deep sub-barrier energies [102].

\subsection{Complex potentials}

The IWBC model leads only to a smooth excitation function for fusion cross sections and does not yield a resonance structure. This is because the incident flux has to return back to the outside of the barrier after it is for a while trapped inside, when a resonance structure is realized. Since the IWBC model assumes a complete absorption of the flux, this resonance behavior does not happen with the IWBC model. While this is a reasonable approximation for medium-heavy 


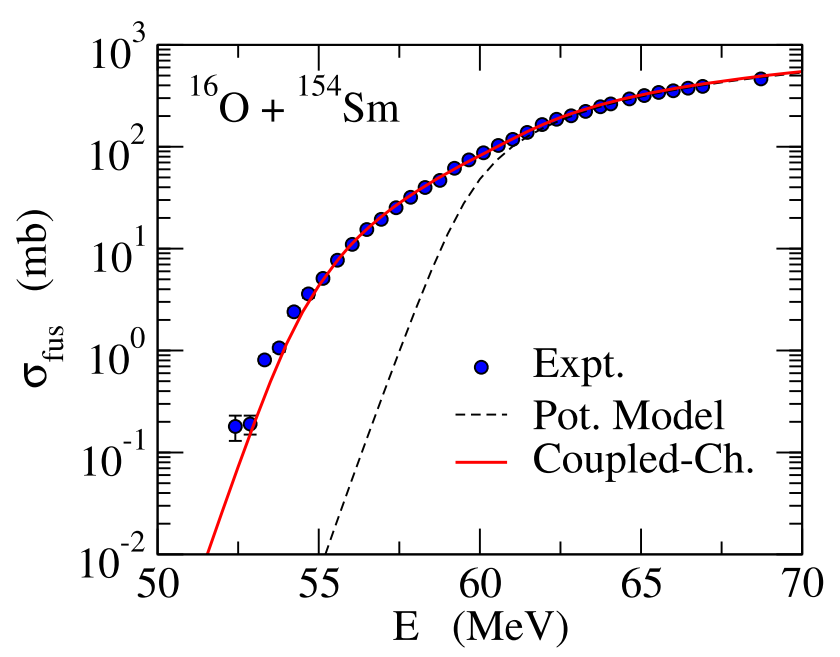

Fig. 13 Comparison between a potential model calculation (the dashed line) and a coupled-channels calculation (the solid line) for fusion cross sections in the ${ }^{16} \mathrm{O}+{ }^{154} \mathrm{Sm}$ system

and heavy systems, light systems often exhibit resonance behaviors in fusion cross sections.

In order to describe such resonances, one would have to use a complex potential with a weak absorption. A typical example is the ${ }^{12} \mathrm{C}+{ }^{12} \mathrm{C}$ reaction, for which pronounced resonance structures have been experimentally observed [15]. The weak absorption is due to the fact that the density of states of the compound system, ${ }^{24} \mathrm{Mg}$, is not large enough, and thus the compound nucleus may not be formed even if the incident flux reaches the inside the Coulomb barrier [103,104]. This situation may be simulated using the imaginary part of an optical potential which depends on the level density, as has been discussed in Refs. [105-108]. In this approach, the imaginary part of the potential is assumed to be

$W(r)=w_{0} \rho_{J}\left(E^{*}\right) f(r)$,

where $w_{0}$ is an overall strength, $\rho_{J}\left(E^{*}\right)$ is the level density of the compound nucleus at the excitation energy of $E^{*}$ with the angular momentum $J$, and $f(r)$ determines the radial dependence of the imaginary potential. This form may be justified if one considers a transition from the entrance channel to compound nucleus states using the Fermi's golden rule [105-108]. In this approach, the energy, the angular momentum, and the system dependences of the imaginary potential are taken into account through the level density of the compound nucleus. It has been demonstrated that the difference between the ${ }^{12} \mathrm{C}+{ }^{12} \mathrm{C}$ and the ${ }^{12} \mathrm{C}+{ }^{13} \mathrm{C}$ systems concerning the resonance structures in the fusion excitation functions can be qualitatively accounted for by this approach [109].

\subsection{Coupled-channels calculations}

As we have briefly discussed in connection with Fig. 12, the large enhancement of fusion cross sections for heavy systems at subbarrier energies can be explained by taking into account the nuclear structure effects of the colliding nuclei. This is to take into account excitations of the colliding nuclei during fusion reactions. This can actually be achieved by using the coupled-channels approach $[4,86]$, in which one solves coupled Schrödinger equations to compute the $S$-matrix. In the case of the ${ }^{16} \mathrm{O}+{ }^{154} \mathrm{Sm}$ system shown in Fig. 12, one has to condider the coupling of the relative motion between the projectile and the target nuclei to the ground state rotational band of ${ }^{154} \mathrm{Sm}$. Such couplings dynamically modify the potential barrier, eventually enhancing fusion cross sections at energies below the static Coulomb barrier. The solid line in Fig. 13 shows fusion cross sections so obtained. One can see that the large enhancement of fusion cross sections can be well accounted for by taking into account the rotational excitations of the ${ }^{154} \mathrm{Sm}$ nucleus. Notice that for such heavy deformed nuclei, the channel coupling effects can be well interpreted in term of angle dependent Coulomb barriers [4].

Incidently, in Ref. [110] Dasgupta et al. underlined the inadequacy of the coherent $\mathrm{CC}$ model because an irreversible energy dissipation starts occurring inside the barrier in this model, but it does not influence the coherence of quantum states. They argued that, instead, an increasing degree of decoherence takes place with increasing overlap of the two nuclei, leading to hindrance of quantum tunneling [111]. the coupled-channels method was extended in Ref. [112] by taking into account the anharmonicity of the multi-octupole phonon states of ${ }^{208} \mathrm{~Pb}$, obtaining better results for the fusion excitation function of ${ }^{16} \mathrm{O}+{ }^{208} \mathrm{~Pb}$, compared to CC calculations in the harmonic-oscillator limit. The barrier distribution is also much better reproduced.

A similar channel coupling can be expected in light systems as well. For instance, it has been realized that a singlechannel optical model calculation discussed in the previous subsection does not yield a sufficient number of subbarrier resonance peaks in the fusion excitation function for the ${ }^{12} \mathrm{C}+{ }^{12} \mathrm{C}$ system. One would then need to extend the potential model by taking into account the excitations in the ${ }^{12} \mathrm{C}$ nuclei. It has been demonstrated that the channel-coupling effects significantly increase the number of resonance peaks in the ${ }^{12} \mathrm{C}+{ }^{12} \mathrm{C}$ system [113]. In fact, several authors have discussed the role of channel couplings in this reaction under different names of the model, that is, the Nogami-Imanishi model [114,115], the double resonance model [116], and the band-crossing model [113]. These models have pointed out the importance of the excitation to the first $2^{+}$state in ${ }^{12} \mathrm{C}$ at $4.44 \mathrm{MeV}$. Thus, when the $2^{+}$state is excited, the relative energy, $E$, decreases by $4.4 \mathrm{MeV}$ and at the same time 
the relative angular momentum is also changed from $l$ to $l$, $l+2$, and $l-2$. If the potential for $l-2$ holds a resonance at $E^{\prime}=E-4.4 \mathrm{MeV}$, this leads to a resonance structure in the fusion excitation function [105]. Notice that this is nothing but the Feshbach resonance, which has been widely discussed in the physics of cold atoms [117,118].

More recent coupled-channels calculations for the ${ }^{12} \mathrm{C}+{ }^{12}$ $\mathrm{C}$ system also include the excitation to the Hoyle state, the second $0^{+}$state at $7.65 \mathrm{MeV}$, as well as the octupole phonon state at $9.64 \mathrm{MeV}$ in ${ }^{12} \mathrm{C}[119,120]$. These calculations have indicated the importance of the inclusion of the Hoyle state and the mutual excitation channels in the coupled-channels calculation for this system.

\subsection{Deep-subbarrier hindrance}

\subsubsection{Theoretical models: sudden versus adiabatic}

For heavy-ion fusion reactions, such as ${ }^{60} \mathrm{Ni}+{ }^{89} \mathrm{Y}$, it has been observed that fusion cross sections fall off much steeper at deep subbarrier energies when compared to a theoretical extrapolation of fusion cross sections based on coupledchannels calculations $[5,11]$.

It was the first pointed out by Brink [121] that the anomaly in the fusion excitation function at extreme sub-barrier energies might be associated with events taking place after passing the barrier, when the densities of projectile and target begin to merge. At this stage, the two-body potential description may fail. Subsequently, Dasso and Pollarolo [122] proposed that a shallow potential is needed to reproduce the experimental cross sections for the fusion of ${ }^{60} \mathrm{Ni}+{ }^{89} \mathrm{Y}$.

In order to interpret the fusion hindrance at extreme subbarrier energies, two theoretical models have been proposed, based either on the sudden approximation by Mişicu and Esbensen [123-125], or on the adiabatic approximation by Ichikawa et al. [126-130]. The difference between the sudden and the adiabatic models is schematically illustrated in Fig. 14. In the sudden model, one usually considers a shallow and thick potential barrier as proposed by Dasso and Pollarolo (see the upper panel of Fig. 14). In Ref. [123,124], a repulsive core is introduced to a double folding potential, Eq. (16), in which the repulsive part is constructed also with the double-folding procedure but with a repulsive zerorange interaction. The strength of the repulsive interaction is determined based on the equation of motion, particularly the incompressibility, $K$, of infinite nuclear matter. The underlying assumption here is that the reaction takes place so suddenly that the density is doubled in the overlapping region of the projectile and the target nuclei. That is, the increase of the potential energy, $\Delta V$, at the origin due to the repulsive core is assumed to be equivalent to the increase of the energy of nuclear matter from the normal density, $\rho_{0}$, to twice the

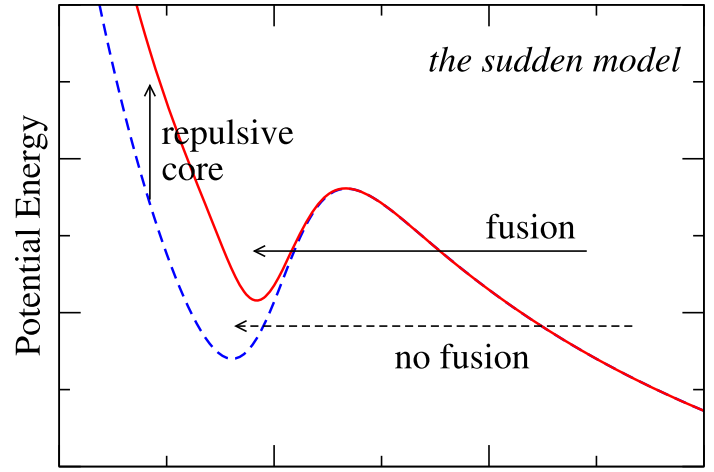

Relative distance

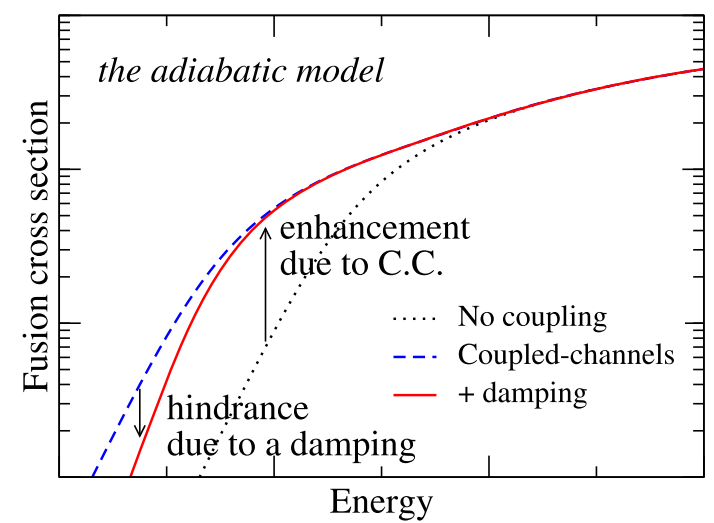

Fig. 14 Schematic illustration of the difference between the sudden model and the adiabatic model for deep-subbarrier fusion hindrance. In the sudden model, fusion hindrance originates from a cut off of high partial waves due to a shallow potential. On the other hand, in the adiabatic model, the hindrance is explained as a consequence of a quenching of channel-coupling effects. The axes are given in arbitrary units

normal density, $2 \rho_{0}$. This leads to the relation $[123,124]$

$\frac{\Delta V}{A_{p}}=\rho_{0}^{2}\left(\frac{\partial^{2} E(\rho)}{\partial \rho^{2}}\right)_{\rho=\rho_{0}}=\frac{K}{9}$,

where $A_{p}$ is the mass number of the projectile nucleus (here the projectile is assumed to be lighter than the target nucleus). In this model, the fusion hindrance takes place because high partial waves are cut off due to the shallow potential.

Notice that the microscopic origin of the repulsion in the overlapping region is due to the Pauli principle, as pointed out in Ref. [125] (see also Refs. [131,132]). In this model, the authors introduced a new microscopic approach to heavy-ion fusion and demonstrated, on the basis of density-constrained frozen Hartree-Fock calculations, that the main effect of Pauli repulsion is to reduce the tunneling probability inside the Coulomb barrier, thus producing the hindrance.

A calculation based on the sudden model is shown in Fig. 15. Here, fusion cross sections (upper panel) and astrophysical $S$-factors (lower panel) are calculated with (solid lines) and without (dotted lines) a repulsive core for the 

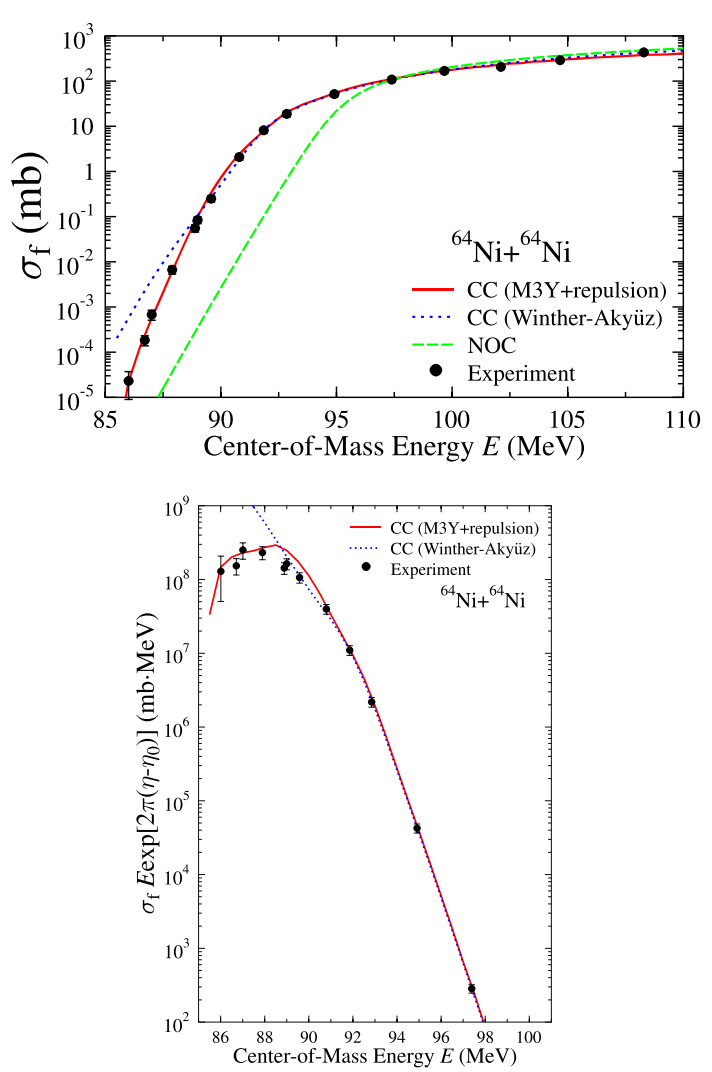

Fig. 15 Fusion cross sections (upper panel) and astrophysical $S$ factors (lower panel) for the ${ }^{64} \mathrm{Ni}+{ }^{64} \mathrm{Ni}$ system. The dotted lines show results of conventional coupled-channels calculations. On the other hand, the solid lines show results of the sudden model with a repulsive core in the internuclear potential. The dashed green curve represents a potential model calculation without couplings. Taken from Ref. [123,124]

${ }^{64} \mathrm{Ni}+{ }^{64} \mathrm{Ni}$ system. One can see that the conventional coupled-channels calculation (dotted lines) works well at energies higher than about $89 \mathrm{MeV}$, but overestimates fusion cross sections, and thus astrophysical $S$-factors, at lower energies. This can be cured by introducing a repulsive core in the internuclear potential, as is demonstrated by the solid lines in the figure.

In contrast to the sudden model, the adiabatic model assumes (see lower panel of Fig. 14) that the internuclear potential is smoothly connected to a mono-nucleus potential, which is often described by the liquid drop model. After touching, this potential is regarded as an adiabatic potential, in which the energy is minimized at each internuclear distance. Since the main effect of excitations has already been taken into account in the adiabatic potential [4], the effect of excitation would be double-counted if one naively continues the coupled-channels calculations even after two nuclei touch each other, although one could still consider a molecular type of excitations. In the adiabatic model, the coupling effect is effectively damped after the touching [126-128].
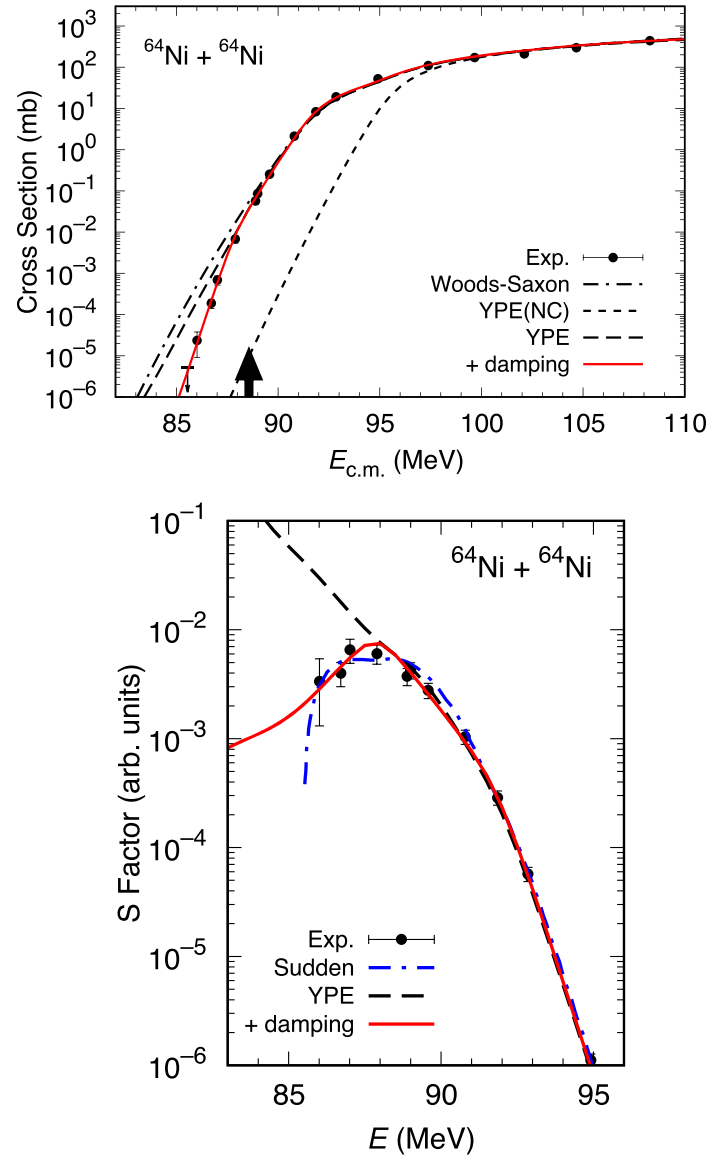

Fig. 16 Similar to (Fig. 15), but with the adiabatic model. Taken from Ref. [128]. Excitation functions obtained from CC calculations using the Woods-Saxon potential as well as calculations using the Yukawaplus-exponential (YPE) without (NC), with couplings and damping are given by short-dashed, long-dashed and solid red curves, respectively. (Courtesey of T. Ichikawa.)

Such gradual damping of collective motions after the touching has been demonstrated microscopically using the random phase approximation (RPA) with two-center shell model wave functions $[129,130]$. In this model, the deep subbarrier hindrance originates from a quenching of an enhancement of fusion cross sections due to the channel coupling effects (see the lower panel of Fig. 14).

Figure 16 shows fusion cross sections (upper panel) and astrophysical $S$ factors (lower panel) obtained with the adiabatic model for the same system as in Fig. 15. One can see that the fit is as good as the fit with the sudden model shown in Fig. 15. This is also the case for other systems [128]. Discriminating between these two approaches would require measuring at even lower energies because only there do the predicted $S$ factors deviate from each other. Such measurements are very interesting, however, would be very challenging as well. Alternatively, one may need observables other than fusion cross sections and astrophysical $S$ factors to judge which model is more reasonable. The mean angular momenta 


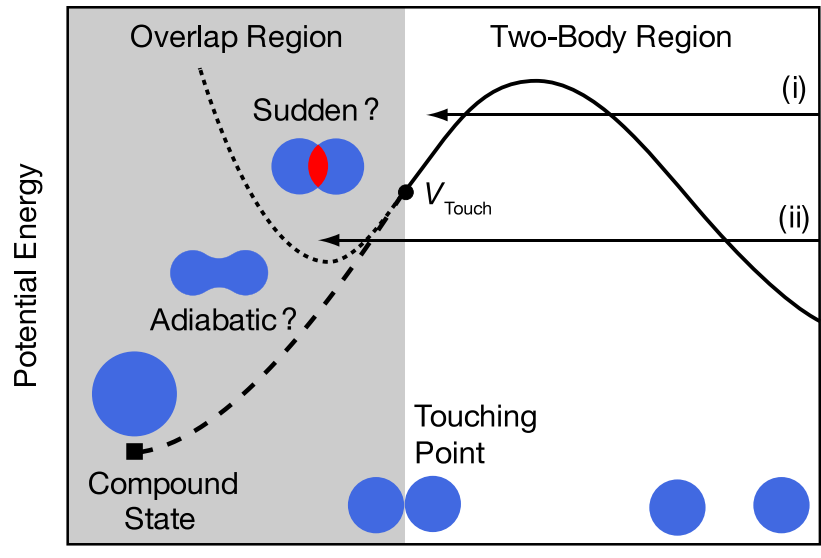

Center-of-Mass Distance $r$

Fig. 17 A schematic illustration of an internucleus potential and the dynamics of fusion reactions in the sudden and the adiabatic models. Taken from Ref. [128]

of a compound nucleus could be used for that purpose [70], even though a direct measurement of such quantity may be extremely challenging at deep subbarrier energies.

We note that, even though the physics origin of the hindrance is different in the sudden and the adiabatic models, both of them point to the importance of dynamical effects after two colliding nuclei touch with each other [133], see Fig. 17 and the note. ${ }^{1}$

\subsubsection{A remark on deep/shallow potentials}

The sudden model appears to correspond to a shallow internuclear potential while the adiabatic model corresponds to a deep potential. We remark that the discussion on the depth of a potential may be slightly more complex. That is, the sudden (the adiabatic) model may not necessarily be connected to a shallow (a deep) potential. First, while it has been widely accepted that the Pauli principle leads to a repulsive core, one may also introduce yet another interpretation of the Pauli principle, by way of the Pauli attraction rather than the Pauli repulsion. Notice that the role of the Pauli principle is to dampen the radial wave function at short distances, and both the Pauli repulsion and the Pauli attraction achieve this in a similar way. This has recently been advocated by Ohkubo for a nucleon-nucleon interaction [134]. The Pauli attraction can naturally emerge from the semi-classical theory in such a way that the physical wave function has to be orthogonalized to Pauli forbidden states and thus the potential has to be deep enough to hold the forbidden states [135-138]. Notice that such deep potentials have to be used with care, as deeply bound states in such a potential would simply be unphysical;

\footnotetext{
1 After submission of the present manuscript, an article by Wen et al. [231] has appeared, which offers further theoretical insight into the sub-barrier hindrance phenomenon.
}

the potential is meaningful only for physical states that are orthogonal to the Pauli forbidden states. Second, those two types of the potentials can be connected to each other using the supersymmetric transformation $[139,140]$. In any case, the potential has to be discussed together with the kinetic energy, otherwise the discussion might be misleading since the potential itself is not an observable.

\subsubsection{Deep subbarrier hindrance in light systems}

An interesting question is whether the deep subbarrier hindrance, which has been observed in medium-heavy systems, still remains in lighter systems [68]. Recent measurements of the ${ }^{12} \mathrm{C}+{ }^{30} \mathrm{Si}[141]$, and ${ }^{12} \mathrm{C}+{ }^{13} \mathrm{C}$ [68] systems have indicated that the hindrance may be absent, or at least rather small, for these light systems. For the ${ }^{12} \mathrm{C}+{ }^{12} \mathrm{C}$ system, theoretical calculations based on the sudden model as well as the density-constrained time-dependent Hartree-Fock method do not show the hindrance effect $[68,102]$. This tendency could be understood naturally with the adiabatic model [126128]. Notice that the strength of the coupling at the barrier is approximately proportional to the charge product of the projectile and the target nuclei, $Z_{1} Z_{2}$, and thus the channel coupling effect is relatively weak in light systems [4]. Therefore, the degree of enhancement of fusion cross sections at subbarrier energies is small. Notice that the adiabatic model explains the origin of the deep-subbarrier hindrance as a consequence of quenching (see Sec. 3.4.1 and the lower panel of Fig. 14). Since the enhancement of the fusion cross section is small in light systems from the beginning, the fusion cross sections are not altered much even if the fusion enhancement is quenched at deep subbarrier energies.

On the other hand, the fusion hindrance may still appear due to the overlap of the two nuclei participating in the collision process. In the sudden model, it is due to the depth of the potential pocket. See Sect. 5 for further discussions on fusion hindrance in light systems.

\section{Fusion Hindrance in medium- and heavy-mass systems}

Fusion excitation functions of medium-mass systems at very low energies are determined by several concurring effects. We have (1) quantum tunneling through the Coulomb barrier, (2) cross section enhancements due to couplings of the entrance channel with low-lying collective modes of the colliding nuclei, producing fusion barrier distributions with various shapes and peak structures, (3) the so-called hindrance phenomenon whose origin is still a matter of debate and experimental work. Fusion hindrance generally shows up below the energy where the barrier distribution vanishes, that is below the energy range where collective enhancements 


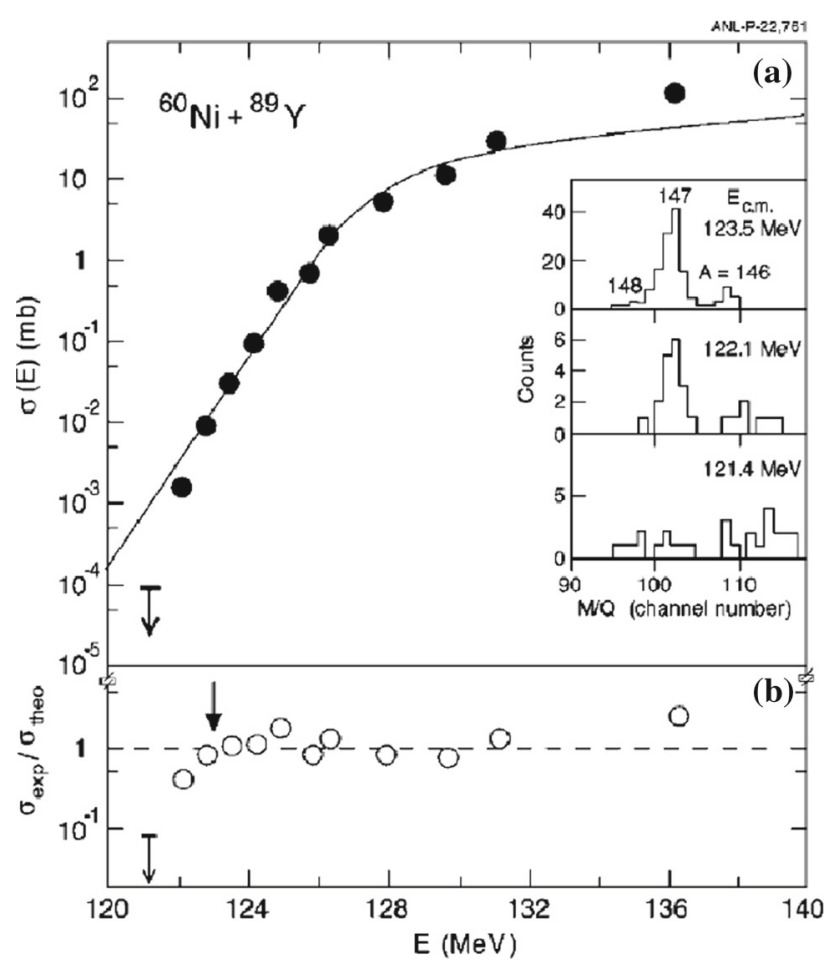

Fig. 18 Fusion excitation function of ${ }^{60} \mathrm{Ni}+{ }^{89} \mathrm{Y}$. Reproduced from the first paper on hindrance [11]

are effective. It is becoming clear that this threshold tends to be lower for systems involving soft nuclei, and/or where couplings to positive $Q$-value nucleon transfer channels are available. Therefore, for such cases hindrance may not be observed if the sensitivity of the experimental set-up is not high enough.

Below we present a few cases where hindrance is known to be present by the shape of the excitation function and/or by the comparison of measured cross sections with theoretical values calculated within standard coupled-channel models.

\subsection{Evidence of hindrance}

Below the lowest barrier produced by channel couplings, one would expect that the energy-weighted excitation functions $E \sigma$ display a simple exponential falloff with decreasing energy [27]. In fact this is not always true, as shown for the first time for the system ${ }^{60} \mathrm{Ni}+{ }^{89} \mathrm{Y}$ by Jiang et al. [11] (see Fig. 18 ), where it was found that, at deep sub-barrier energies, the cross section decreases very rapidly, much steeper than predicted by a simple exponential falloff [27]. This fusion cross section was measured down to $1.6 \mu \mathrm{b}$ (an upper limit of $95 \mathrm{nb}$ was established for the lowest measured energy) using the Fragment Mass Analyzer [38] at ATLAS.

Other systems investigated in the same energy range far below the barrier gave evidence that the slope of many excitation functions keeps increasing with decreasing energy. It is clear by now that the low-energy hindrance effect is a general phenomenon for heavy-ion fusion, even if it shows up with varying intensities and distinct features in different systems.

Fusion hindrance is often conveniently represented by the logarithmic slope $L(E)$ of the excitation function or by the astrophysical $S$ factor $S(E)$ [29] defined by Eqs. (7) and (6).

An $S$-factor maximum occurs at the point where the logarithmic derivative $L(E)$ reaches the value $\frac{\pi \eta}{E}$. Since the two quantities $L(E)$ and $S(E)$ are algebraically related [13]

$\frac{d S}{d E}=S(E)\left[L(E)-\frac{\pi \eta}{E}\right]$,

the derivative, $d S / d E$, vanishes when the logarithmic derivative equals $\pi \eta / E$, and an $S$-factor maximum appears. At this point, the energy and logarithmic derivative values are denoted as $E_{S}$ and $L_{s}$, respectively, and the quantity $L_{c S}(E)=\frac{\pi \eta}{E}$ is called the constant $S$-factor function. The condition for an $S$-factor maximum thus leads to the relation

$E_{s}=\left[0.495 \zeta / L_{S}\right]^{2 / 3}(\mathrm{MeV})$,

where $E_{s}$ and $L_{s}$ are given in units of $\mathrm{MeV}$ and $1 / \mathrm{MeV}$, respectively, and $\zeta$ is a dimensionless system parameter

$\zeta=Z_{1} Z_{2} \sqrt{\frac{M_{1} M_{2}}{M_{1}+M_{2}}}$,

where $Z_{1}$ and $Z_{2}$ are the respective charge numbers and $M_{1}$ and $M_{2}$ are the respective nuclear masses in units of $M_{N}, M_{N}$ being the nucleon mass. The behavior of the excitation function in the energy region near and below the barrier is usually well described by the $S$ factor. $S(E)$ can be extracted directly from the cross section, whereas the logarithmic slope $L(E)$, being a derivative of the excitation function, is subject to larger experimental uncertainties.

Historically, the $S(E)$ representation has frequently been used in light-ion reactions, where the Gamow factor $\exp (-2 \pi \eta)$ accounts for the main part of the strong energy dependence of the fusion cross sections. As a consequence, far below the barrier the $S$ factor has only a weak dependence on energy for proton- and $\alpha$-induced reactions. The $S$ factor for heavy-ion fusion reactions, however, has a very strong energy dependence just below the Coulomb barrier; it increases steeply with decreasing energy, reflecting the weak energy dependence of the $E \sigma$ product when compared to that of the Gamow factor.

Nevertheless, when the fusion $Q$ value is negative, S must have a maximum because it has to drop to zero at the positive center-of-mass energy $E=-Q$, where the ground state of the compound nucleus is reached. That is

$S(E) \rightarrow 0$ for $E \rightarrow-Q$ when $Q<0$.

The energy where $L(E)=L_{c s}(E)=\pi \eta / E$ (and the $S$ factor has a maximum) has often been taken as the threshold for the hindrance effect. However, we will see that hindrance 


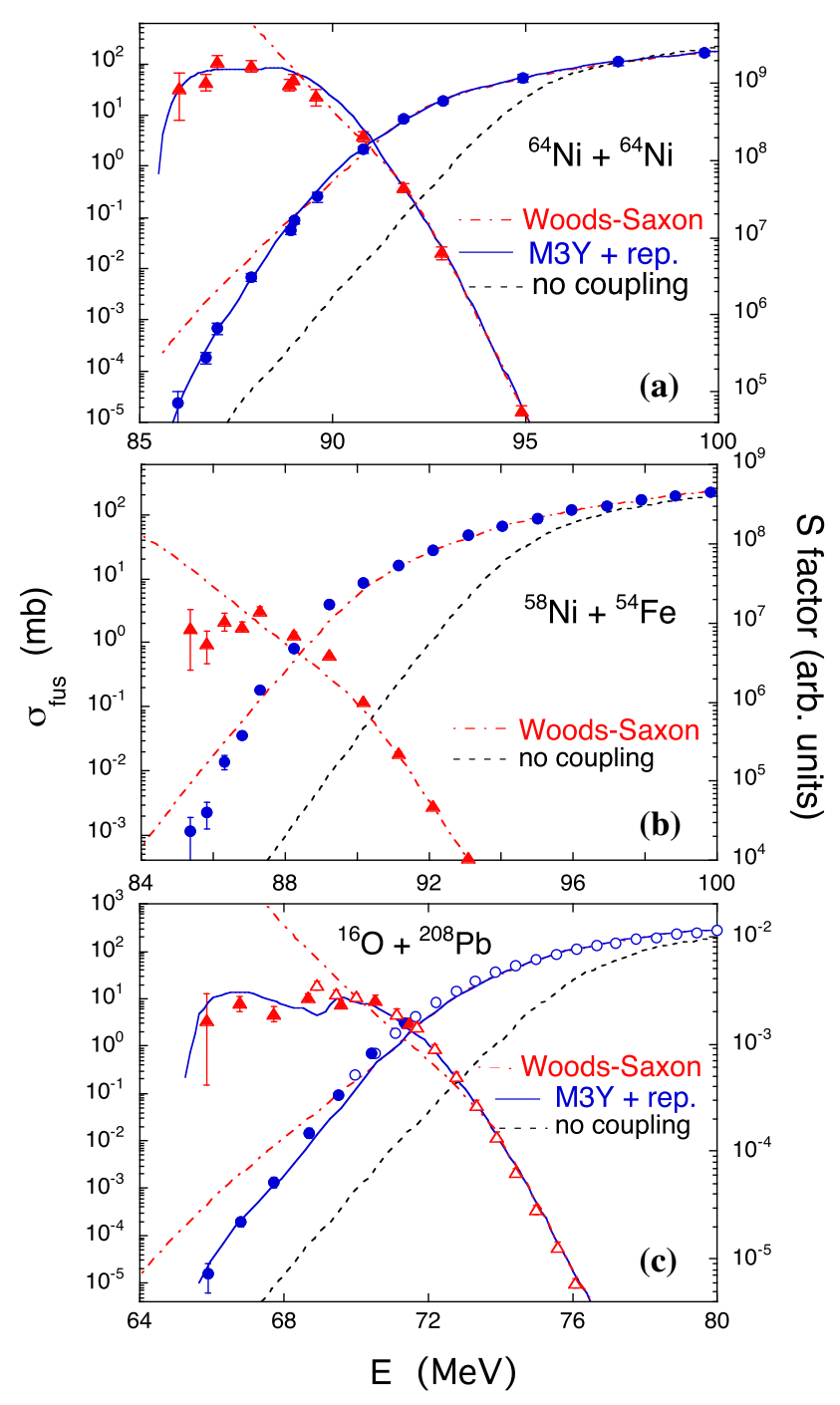

Fig. 19 Fusion excitation functions and astrophysical $S$ factors of ${ }^{64} \mathrm{Ni}+{ }^{64} \mathrm{Ni}[12](\mathbf{a}),{ }^{58} \mathrm{Ni}+{ }^{54} \mathrm{Fe}[46](\mathbf{b})$ and ${ }^{16} \mathrm{O}+{ }^{208} \mathrm{~Pb}(\mathbf{c})$, compared to $\mathrm{CC}$ calculations. For the last system, full symbols refer to the more recent measurement from Ref. [110], while the open symbols originate from Morton et al. [142]

may show up even in the absence of a maximum of $S(E)$. Conversely, for systems whose fusion $Q$ value is positive, it is not necessary to have an $S$ factor maximum, although there could be one.

\subsubsection{Examples of the fusion hindrance}

In Fig. 19 we show the fusion hindrance for three representative cases: (a) ${ }^{64} \mathrm{Ni}+{ }^{64} \mathrm{Ni}$ [12], (b) ${ }^{58} \mathrm{Ni}+{ }^{54} \mathrm{Fe}$ [46], and (c) ${ }^{16} \mathrm{O}+{ }^{208} \mathrm{~Pb}[110,142]$. The fusion excitation function for ${ }^{64} \mathrm{Ni}+{ }^{64} \mathrm{Ni}$ was measured down to $\simeq 20 \mathrm{nb}$. The cross sections far below the barrier are much lower than predicted by standard CC calculations employing the Woods-Saxon potential shown by the red dash-dotted line. The $S$ factor data are shown by red symbols. Below the Coulomb barrier, the $S$ factor develops a clear maximum. The WS calculation starts overpredicting the excitation function below $\sim 89$ $\mathrm{MeV}$ indicating the onset of fusion hindrance. The $\mathrm{CC}$ calculation using the $\mathrm{M} 3 \mathrm{Y}+$ repulsion potential (blue continuous line) gives a good account of the data.

Similar results are obtained for the systems ${ }^{58} \mathrm{Ni}+{ }^{54} \mathrm{Fe}$ (b) and ${ }^{16} \mathrm{O}+{ }^{208} \mathrm{~Pb}$ (c). The calculations based on a WS potential overpredict the low energy part of the excitation function in both cases, and a maximum of the $S$ factor vs. energy appears.

For ${ }^{58} \mathrm{Ni}+{ }^{54} \mathrm{Fe}$ it is observed that the $S$ factor maximum shows up at an energy where the fusion cross section is larger when compared to ${ }^{64} \mathrm{Ni}+{ }^{64} \mathrm{Ni}$. This may be a consequence of the different structure of the nuclei involved in the reaction; ${ }^{58} \mathrm{Ni}$ and ${ }^{54} \mathrm{Fe}$ are stiff, whereas ${ }^{64} \mathrm{Ni}$ is softer. This will be discussed in more detail below.

For ${ }^{16} \mathrm{O}+{ }^{208} \mathrm{~Pb}$, the previous data of Morton et al. [142] were augmented by Dasgupta et al. [110] and Fig. 19c shows the complete excitation function, where the low-energy slope appears to be very steep. The astrophysical $S$ factor saturates in the same energy range. The calculations based on a WS potential overpredict the low-energy part of the excitation function, whereas the $\mathrm{CC}$ calculations using the M3Y + repulsion potential closely fit the low-energy cross sections and the $\mathrm{S}$ factor.

Another case involves the system ${ }^{64} \mathrm{Ni}+{ }^{100}$ Mo [143], whose fusion behavior is shown in Fig. 20 in terms of the fusion excitation function and its logarithmic derivative. In this case, CC calculations with a WS potential reproduce the data reasonably well with the low-energy cross sections mainly determined by the strong quadrupole vibration of ${ }^{100} \mathrm{Mo}$. Up to four phonons of this collective mode have to be considered; however, one notices that the lowest energy points decrease faster than the $\mathrm{CC}$ results. This is better seen in a plot of the logarithmic slope (red triangles) showing that the $\mathrm{CC}$ calculations tend to saturate below the barrier, while the experimental points keep increasing and the logarithmic slope reaches the $L_{c s}$ value, though only in the sub- $\mu$ b cross section range.

We would like to point out the different behavior between ${ }^{58} \mathrm{Ni}+{ }^{54} \mathrm{Fe}$, where fusion hindrance shows up clearly, and the case of ${ }^{48} \mathrm{Ti}+{ }^{58} \mathrm{Fe}$ [144], where fusion hindrance is not observed in the measured energy range down to $\simeq 2 \mu \mathrm{b}$. As discussed in Ref. [6] (see Fig. 39 of that article), the logarithmic slope of ${ }^{58} \mathrm{Ni}+{ }^{54} \mathrm{Fe}$ below the barrier keeps increasing, reaching and exceeding the value $L_{c s}$ [12], while the slope of ${ }^{48} \mathrm{Ti}+{ }^{58} \mathrm{Fe}$ saturates and stays much lower than $L_{c s}$.

The situation is shown in Fig. 21, where panel (b) demonstrates the different trends of the two $S$ factors, compared to standard CC calculations using a WS potential and including the $2^{+}$and $3^{-}$states of the nuclei. It is also interesting to 


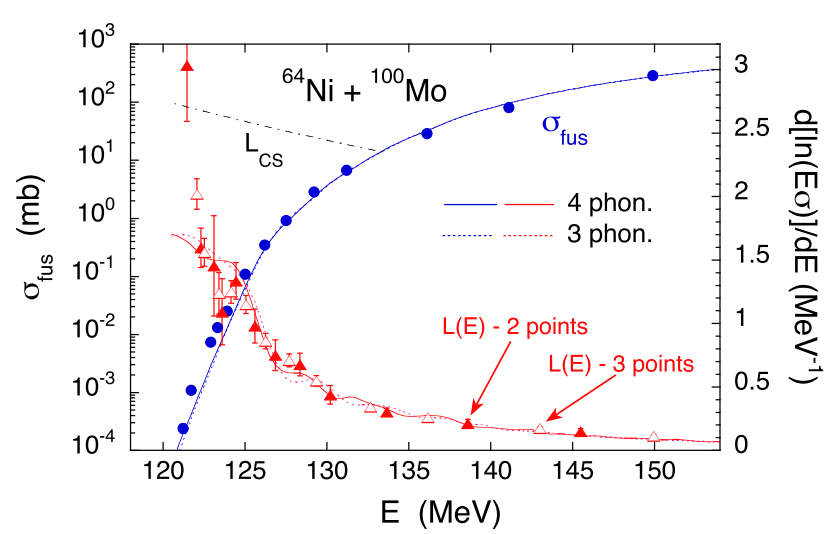

Fig. 20 Fusion excitation function (left scale) and logarithmic derivative (right scale) of ${ }^{64} \mathrm{Ni}+{ }^{100} \mathrm{Mo}$ [143] compared to CC calculations (see text for detail)

examine the two barrier distributions (BD) shown in panel (a) of the same figure. The energy scale of the plot is normalized to the Akyüz-Winther barrier [150]. Both distributions have a complex structure with several peaks. However, the $\mathrm{BD}$ of ${ }^{48} \mathrm{Ti}+{ }^{58} \mathrm{Fe}$ is wider at low energies and extends lower with respect to $\mathrm{V}_{b}$ than for ${ }^{58} \mathrm{Ni}+{ }^{54} \mathrm{Fe}$ pushing the hindrance threshold for this system below the investigated energy range.

The difference in nuclear structure of the colliding nuclei gives rise to this situation, because ${ }^{48} \mathrm{Ti}$ and ${ }^{58} \mathrm{Fe}$ are soft nuclei with rather strong and low-lying quadrupole excitations at $\approx 800-900 \mathrm{keV}$, while ${ }^{58} \mathrm{Ni}$ and ${ }^{54} \mathrm{Fe}$ have closed proton and neutron shells and are rather stiff. The stiffness of the reaction partners thus allows the fusion hindrance to appear already at a level of $\approx 180 \mu \mathrm{b}$.

\subsection{Influence of transfer channels and low-energy excitations}

Several studies have given firm evidence that the excitation of low-lying collective states $(Q<0)$ and the nucleon transfer reactions, especially those channels having $Q>0$ (Broglia et al. [145]), are the two most important contributions to the near-barrier fusion enhancement.

The CC model $[7,8]$ indicates that coupling to $Q>0$ reaction channels produces a change of the sub-barrier excitation function different from what is expected by inelastic couplings with $Q<0$ because the excitation function decreases more slowly below the barrier when $Q>0$ due to the different shapes of the barrier distribution produced by the two kinds of couplings.

\subsubsection{The Ca + Zr systems}

The case of ${ }^{40} \mathrm{Ca}+{ }^{96} \mathrm{Zr}$ [146] is of particular interest for the investigation of the effects of transfer couplings on fusion, because the $Q$-values for g.s. $\rightarrow$ g.s. neutron pick-up transfer
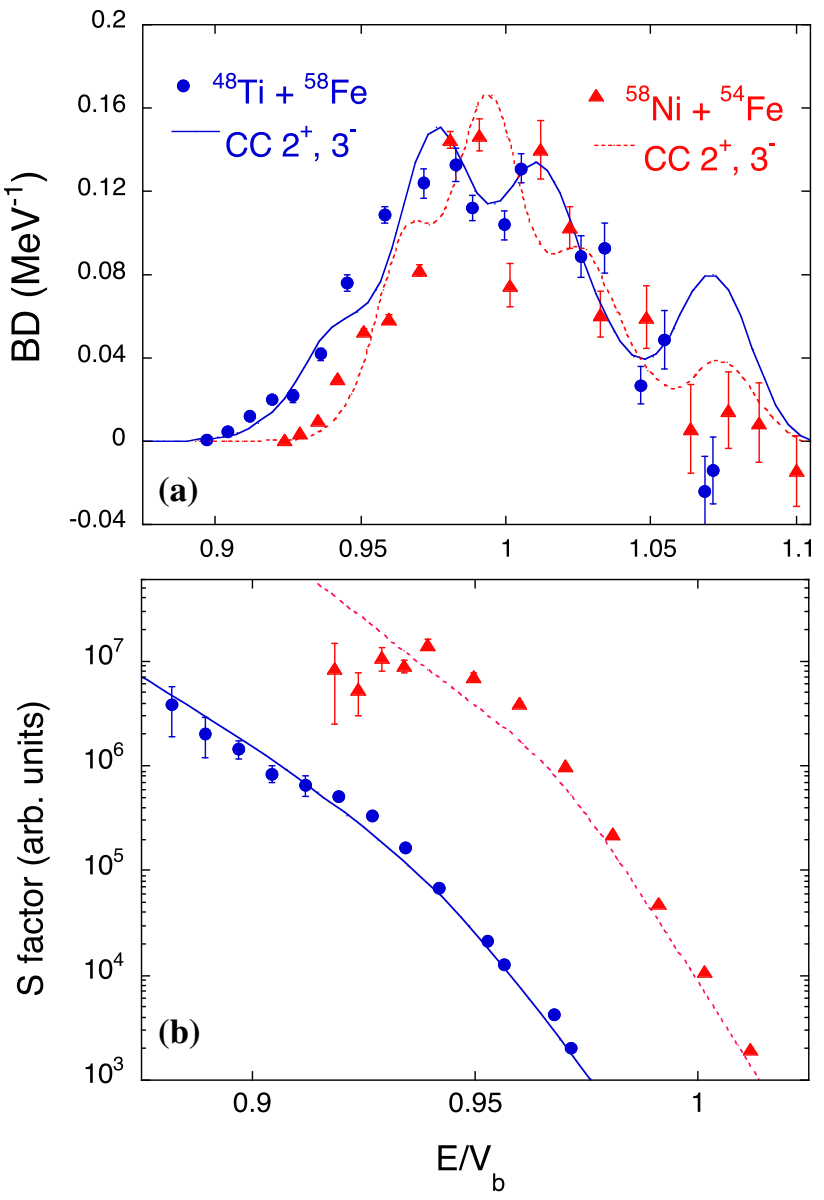

Fig. 21 Fusion barrier distributions (BD) (a) and $S$ factors of ${ }^{48} \mathrm{Ti}+{ }^{58}$ Fe and ${ }^{58} \mathrm{Ni}+{ }^{54} \mathrm{Fe}(\mathbf{b})$ compared to standard $\mathrm{CC}$ calculations using a Woods-Saxon potential. $V_{b}$ are 73.26 and $92.93 \mathrm{MeV}$ for ${ }^{48} \mathrm{Ti}+{ }^{58} \mathrm{Fe}$ and ${ }^{58} \mathrm{Ni}+{ }^{54} \mathrm{Fe}$, respectivly

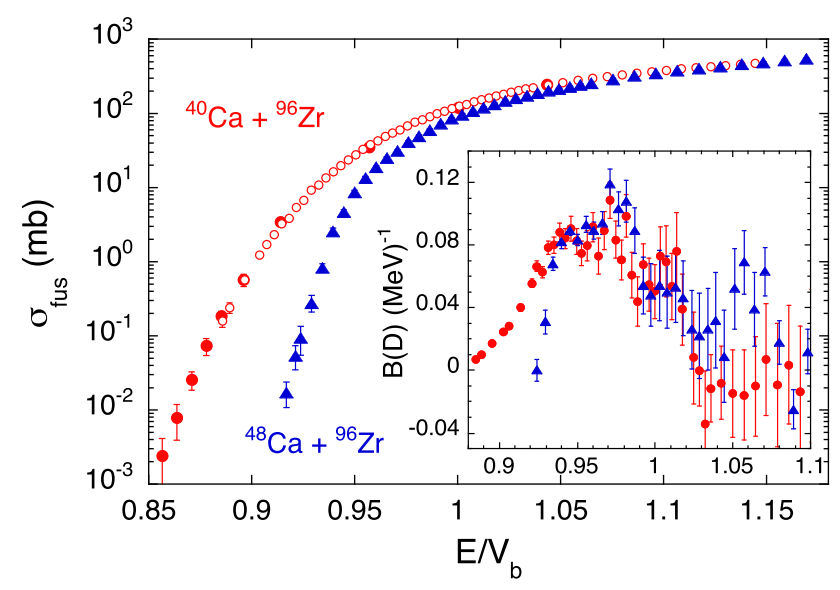

Fig. 22 Fusion excitation functions of ${ }^{40} \mathrm{Ca}+{ }^{96} \mathrm{Zr}$ (full dots from Ref. [146], open symbols from Ref. [147,148]) and triangles for ${ }^{48} \mathrm{Ca}+{ }^{96} \mathrm{Zr}$ [149]. The insert shows the barrier distributions of the two systems. The energy scale is relative to the Coulomb barrier $\mathrm{V}_{b}$ obtained with the Akyüz Winther potential [150]. The values of $V_{b}$ are 98.30 and 95.90 $\mathrm{MeV}$ for ${ }^{40} \mathrm{Ca}+{ }^{96} \mathrm{Zr}$ and ${ }^{48} \mathrm{Ca}+{ }^{96} \mathrm{Zr}$, respectivly 


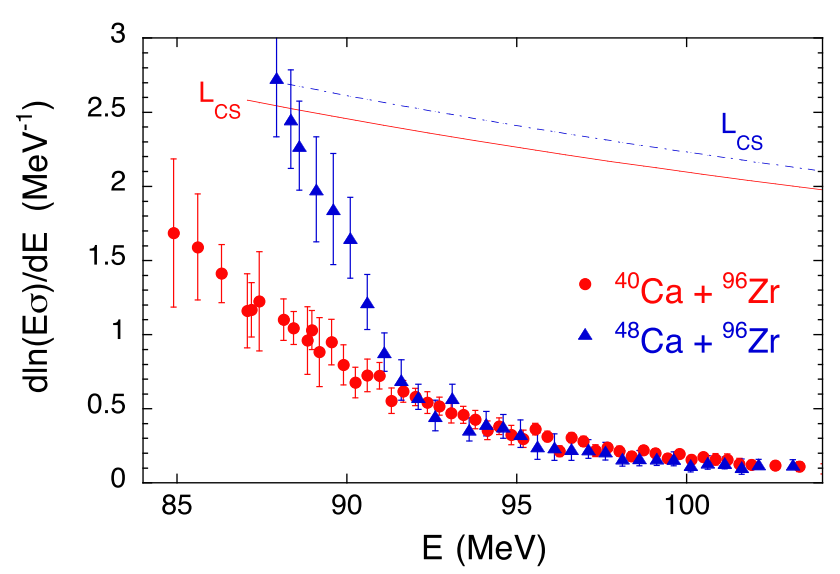

Fig. 23 Logarithmic slopes for the two systems ${ }^{40} \mathrm{Ca}+{ }^{96} \mathrm{Zr}[146-$ 148] (red dots) and ${ }^{48} \mathrm{Ca}+{ }^{96} \mathrm{Zr}$ [149] (blue triangles). $L(E)$ values were obtained using the two-points difference method

channels are very large and positive, i.e. $Q=+5.53,+9.64$ and $+11.62 \mathrm{MeV}$ for $2 n, 4 n$ and $6 n$ transfers, respectively.

The sub-barrier fusion excitation function for this reaction has been measured down to cross sections as small as $\simeq$ $2.4 \mu \mathrm{b}$, two orders of magnitude smaller than obtained in a previous experiment $[147,148]$. The low-energy fusion cross section was found to be greatly enhanced with respect to ${ }^{40} \mathrm{Ca}+{ }^{90} \mathrm{Zr}$, and the need of coupling to transfer channels was suggested.

The comparison with ${ }^{48} \mathrm{Ca}+{ }^{96} \mathrm{Zr}$, where no $Q>0$ transfer channels are available, is very informative (see Fig. 22). The sub-barrier cross section for this system drops very steeply, while the excitation function of ${ }^{40} \mathrm{Ca}+{ }^{96} \mathrm{Zr}$ decreases slowly (and smoothly) below the barrier. From the insert of the same figure, we note that the two barrier distributions have quite different shapes, that of ${ }^{40} \mathrm{Ca}+{ }^{96} \mathrm{Zr}$ extending much further toward low energies. This leads to a flatter slope of the excitation function of this system, and indicates the effect of nucleon transfer.

The experimental logarithmic slopes of the two systems are shown in Fig. 23. One notices the very different behavior of the two systems where the steep sub-barrier slope of ${ }^{48} \mathrm{Ca}+{ }^{96} \mathrm{Zr}$, leading to hindrance, is not observed for ${ }^{40} \mathrm{Ca}+{ }^{96} \mathrm{Zr}$ whose excitation function decreases very slowly below the barrier. Indeed, the wider $\mathrm{BD}$ for ${ }^{40} \mathrm{Ca}+{ }^{96} \mathrm{Zr}$ probably pushes the onset of the hindrance effect to lower energies.

The recent CC analysis [151] of the excitation function for ${ }^{40} \mathrm{Ca}+{ }^{96} \mathrm{Zr}$ is shown in Fig. 24. It includes explicitly one- and two-nucleon $Q>0$ transfer channels with coupling strengths calibrated to reproduce the measured neutrontransfer data. Such transfer couplings give rise to significant cross section enhancements, even at the level of a few $\mu \mathrm{b}$. One obtains an excellent account of the fusion data; a significant contribution to the enhancement is due also to proton stripping channels having positive $Q$-values as well.

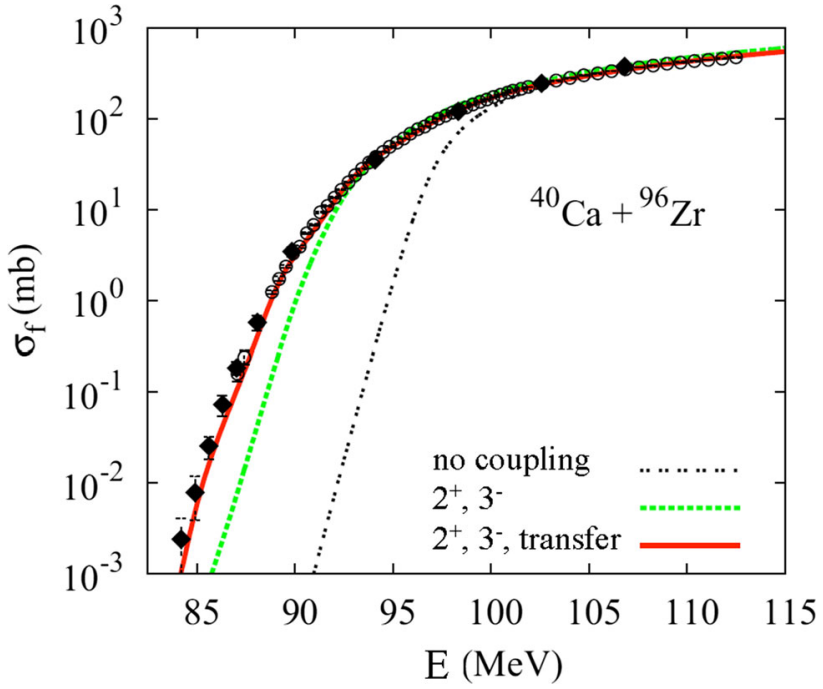

Fig. 24 Fusion cross sections for ${ }^{40} \mathrm{Ca}+{ }^{96} \mathrm{Zr}$ [146-148] are compared with CC calculations [151] using the WS potential. The red full line reproducing the data includes couplings to the one- and two-nucleon transfer channels as well as the inelastic $2^{+}$and $3^{-}$excitations in both nuclei (dotted green line). Figure adapted from [151]

The hindrance caused by Pauli blocking is suppressed in ${ }^{40} \mathrm{Ca}+{ }^{96} \mathrm{Zr}$ by the large number of transfer channels with positive $Q$-values [151]. Locating the hindrance threshold in ${ }^{40} \mathrm{Ca}+{ }^{96} \mathrm{Zr}$ would require challenging measurements of cross sections in the sub- $\mu$ b range.

\subsubsection{The Ni $+\mathrm{Ni}$ systems and other cases}

In the ${ }^{58} \mathrm{Ni}+{ }^{64} \mathrm{Ni}$ system, the influence of positive $Q$-value transfer channels on near-barrier fusion was demonstrated by Beckerman et al. [16]. For this case, the lowest measured cross section was relatively large $(\sigma \simeq 0.1 \mathrm{mb}$ ). The fusion excitation function of ${ }^{58} \mathrm{Ni}+{ }^{64} \mathrm{Ni}$ has recently been remeasured and extended to lower cross sections by two orders of magnitude in experiments performed at the XTU Tandem accelerator of LNL [152].

The case of ${ }^{58} \mathrm{Ni}+{ }^{64} \mathrm{Ni}$ is very similar to ${ }^{40} \mathrm{Ca}+{ }^{96} \mathrm{Zr}$ because of the gentle fall-off of both sub-barrier fusion excitation functions, originating from the couplings to several $Q>0$ neutron pick-up channels.

The new results for ${ }^{58} \mathrm{Ni}+{ }^{64} \mathrm{Ni}$ are in agreement with previous data [16] and are shown in the upper panel of the Fig. 25 (blue dots) compared to the previous results for the symmetric $\mathrm{Ni}+\mathrm{Ni}$ systems $[12,16]$. We notice that the gentle fall-off of the sub-barrier cross sections for ${ }^{58} \mathrm{Ni}+{ }^{64} \mathrm{Ni}$ continues down to the level of $\simeq 1 \mu \mathrm{b}$. The fusion excitation functions of ${ }^{58} \mathrm{Ni}+{ }^{64} \mathrm{Ni}$ and ${ }^{64} \mathrm{Ni}+{ }^{64} \mathrm{Ni}$ are compared in the lower panel of the figure with the results of coupled-channels calculations. While for ${ }^{64} \mathrm{Ni}+{ }^{64} \mathrm{Ni}$ the low energy data are overpredicted by a standard Woods-Saxon CC calculation 


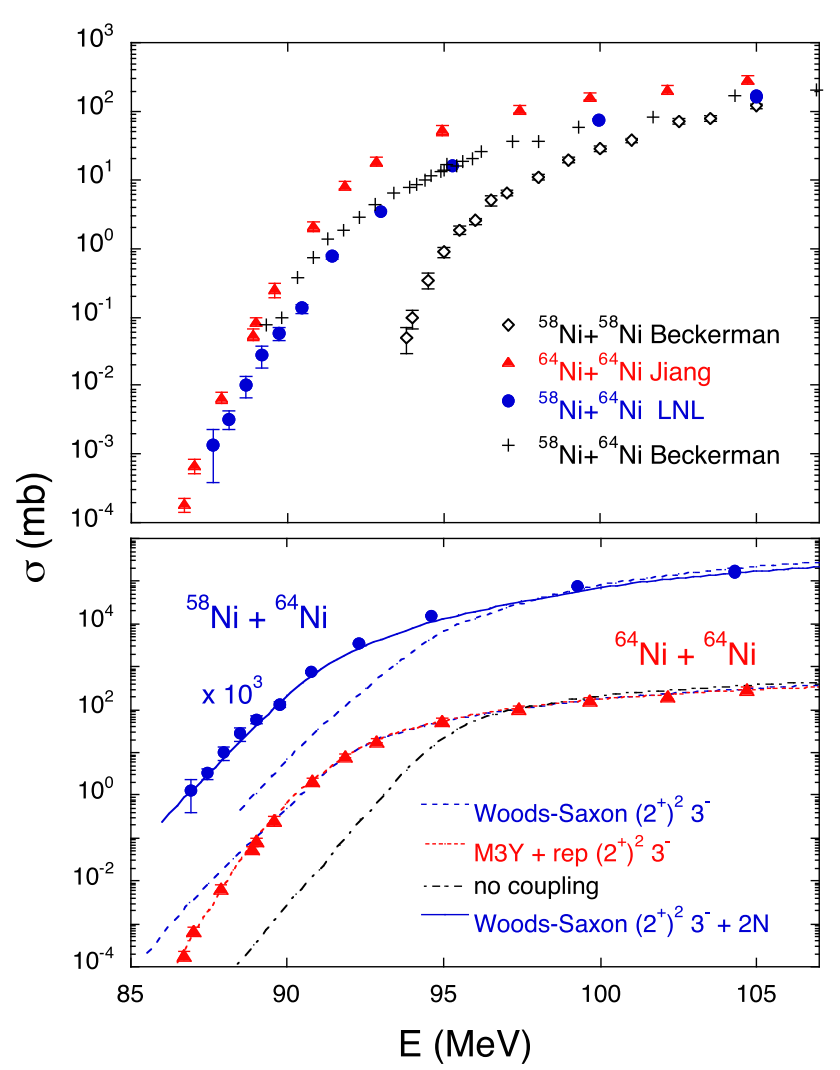

Fig. 25 (upper panel) Fusion cross sections of various $\mathrm{Ni}+\mathrm{Ni}$ systems. (lower panel) Experimental data of ${ }^{58} \mathrm{Ni}+{ }^{64} \mathrm{Ni},{ }^{64} \mathrm{Ni}$ compared to $\mathrm{CC}$ calculations (see text for details)

and one needs a $\mathrm{M} 3 \mathrm{Y}+$ repulsion potential for a good fit (see Fig. 19), the low-energy cross sections of ${ }^{58} \mathrm{Ni}+{ }^{64} \mathrm{Ni}$ are underpredicted by an analogous Woods-Saxon calculation.

This trend at far sub-barrier energies (no hindrance observed for ${ }^{58} \mathrm{Ni}+{ }^{64} \mathrm{Ni}$ ) suggests that, as was observed for ${ }^{40} \mathrm{Ca}+{ }^{96} \mathrm{Zr}$, the availability of several states following transfer with $Q>0$ effectively counterbalances the Pauli repulsion that, in general, is predicted to reduce the tunneling probability through the Coulomb barrier $[125,153]$.

We should also mention that the two systems ${ }^{35,37} \mathrm{Cl}+$ ${ }^{130} \mathrm{Te}$ have recently been investigated at HYRA $[154,155]$. The evidence coming from those experiments points at the importance of the two-neutron pick-up channel for fusion of ${ }^{35} \mathrm{Cl}+{ }^{130} \mathrm{Te}$, as opposed to the case of ${ }^{37} \mathrm{Cl}+{ }^{130} \mathrm{Te}$, where that channel with a positive $Q$-value is not available. Also, a universal correlation between the fusion excitation function and the strength of the total neutron-transfer cross sections for systems ranging from $\mathrm{S}+\mathrm{Ca}$ to $\mathrm{Ni}+\mathrm{Sn}$ has been studied in Ref. [156].

No $S$-factor maximum has so far been observed in systems having a negative fusion $Q$ value and strong $Q>0$ transfer couplings, because in these cases the maximum probably shows up at very low energies.

\subsubsection{Low-energy excitation modes}

It has been shown in some representative examples discussed in the previous paragraphs, that fusion hindrance is in general easier to detect in fusion reactions between stiff nuclei, as are most of the cases presented above. Indeed, systems where soft nuclei are involved generally show a similar behavior, as far as fusion hindrance is concerned, to the cases we have discussed above having positive $Q$-value nucleon transfer channels. This happens because fusion barrier distributions of soft systems tend to be very wide due to the effect of strong couplings to collective modes of excitation. Consequently, the threshold of hindrance ( $S$-factor maximum), that shows up where the barrier distribution vanishes, may be pushed down in energy, and possibly becomes difficult to reach, depending on the sensitivity of the set-up that has to be able to measure very small cross sections at deep sub-barrier energies.

Let us consider again the system ${ }^{64} \mathrm{Ni}+{ }^{100}$ Mo [143] where hindrance shows up at very low sub-barrier energies due to the soft structure of the two nuclei and, in particular, because of the strong quadrupole vibration of ${ }^{100} \mathrm{Mo}$ (see Fig. 20). In Fig. 26, we show the behavior of the near-by case ${ }^{60} \mathrm{Ni}+{ }^{100} \mathrm{Mo}$ [157], where the presence of various neutron transfer channels with $Q>0$ could influence the sub-barrier cross sections. Actually, the quadrupole vibration of ${ }^{100} \mathrm{Mo}$ is the dominant ingredient for enhancement also in this case. Figure 26 illustrates this situation, in which the excitation function is well reproduced by $\mathrm{CC}$ calculations including that vibration up to the 4 th phonon level. However the lowest energy points are slightly under-predicted, and the slope $L(E)$ does not increase so much below the barrier (see the insert), as was noticed for ${ }^{64} \mathrm{Ni}+{ }^{100}$ Mo. Hindrance is not observed in ${ }^{60} \mathrm{Ni}+{ }^{100}$ Mo down to level of $\simeq 2 \mu \mathrm{b}$ and this may very well be a consequence of the $Q>0$ neutron pickup channels, as was suggested in Ref. [157]. A small relative enhancement of ${ }^{60} \mathrm{Ni}+{ }^{100}$ Mo with respect to ${ }^{64} \mathrm{Ni}+{ }^{100} \mathrm{Mo}$ was indeed observed (see Fig. 2 of the original article) at low energies.

For such heavy and soft systems, in general, fusion is strongly affected by multi-phonon excitations, so that one should include couplings to all orders in the CC calculations, and the simple harmonic approximation of the vibrational modes should not be used. It is, however, unfortunate that in most cases the experimental information on multi-phonon states is missing.

During the initial studies of fusion hindrance, a phenomenological analysis led to a purely empirical formula [158] for the expected energy $E_{s}^{r e f}$ of the $S$-factor maximum. The formula was originally developed for medium-mass systems with negative fusion $Q$-value, involving closed-shell nuclei for both projectile and target (stiff systems). It was found that the logarithmic derivative of the energy-weighted 


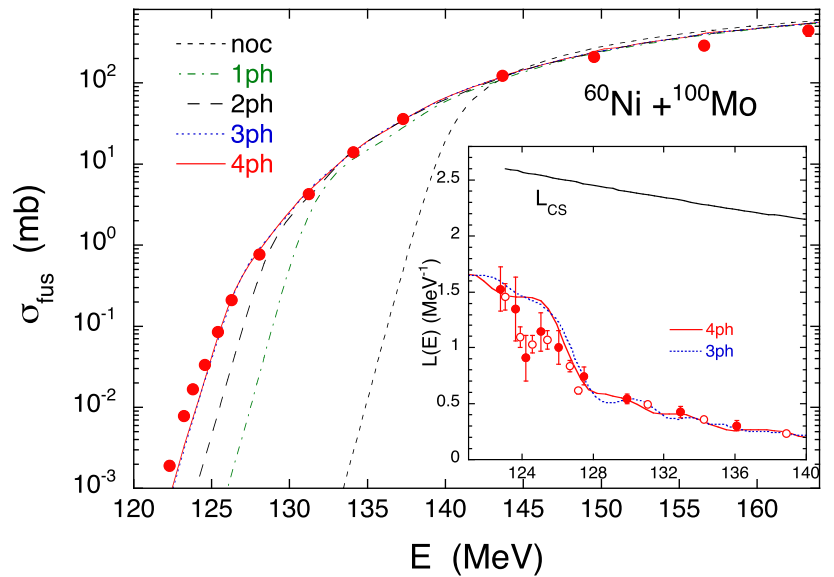

Fig. 26 Fusion excitation function of ${ }^{60} \mathrm{Ni}+{ }^{100} \mathrm{Mo}$ [157] compared with $\mathrm{CC}$ calculations using the WS potential and including up to four phonons of the quadrupole vibration in ${ }^{100} \mathrm{Mo}$. The inset shows the logarithmic derivative, $L(E)$ obtained using all data point (full dots) and every second one (open dots)

cross section, $L_{s}$, at the energy $E_{s}^{r e f}$, has the nearly constant value $L_{s}^{r e f} \sim 2.33 \mathrm{MeV}^{-1}$.

The condition for the $\mathrm{S}$-factor maximum is $L_{s}^{\text {ref }}=$ $\pi \eta / E_{s}^{r e f}$ (see Eq. 19). The Sommerfeld parameter, Eq. 8, is given by

$$
\begin{aligned}
\eta & =Z_{1} Z_{2}^{2} /(\hbar v)=0.1575 \frac{Z_{1} Z_{2}}{\sqrt{E}} \sqrt{\frac{M_{1} M_{2}}{M_{1}+M_{2}}} \\
& =0.1575 \frac{\zeta}{\sqrt{E}},
\end{aligned}
$$

where $\mathrm{E}$ is the center-of-mass energy given in units of $\mathrm{MeV}$. Using the condition for the $S$-factor maximum, $L_{s}=\pi \eta / E_{s}$, we thus obtain

$E_{s}^{r e f}=0.356\left[Z_{1} Z_{2} \sqrt{\frac{M_{1} M_{2}}{M_{1}+M_{2}}}\right]^{2 / 3}=0.356 \zeta^{2 / 3}(\mathrm{MeV})$.

It was found that the energy, $E_{S}$, of the $S$-factor maximum tends to decrease with respect to $E_{s}^{r e f}$, when the total number of "valence nucleons" outside closed shells in the entrance channel, increases [157].

A quantitative relation between the stiffness and the deviation from $E_{S}$ has not yet been established. The fusion hindrance for soft systems occurs at center-of-mass energies $E_{s}$ that are 7-15 MeV lower than the systematics established for the stiff systems.

\subsubsection{The Si + Si systems: isotopic effects far below the barrier}

The fusion excitation function for ${ }^{28} \mathrm{Si}+{ }^{28} \mathrm{Si}$ has recently been measured down to a level of $\simeq 600 \mathrm{nb}$ [159] (see Fig. 27).

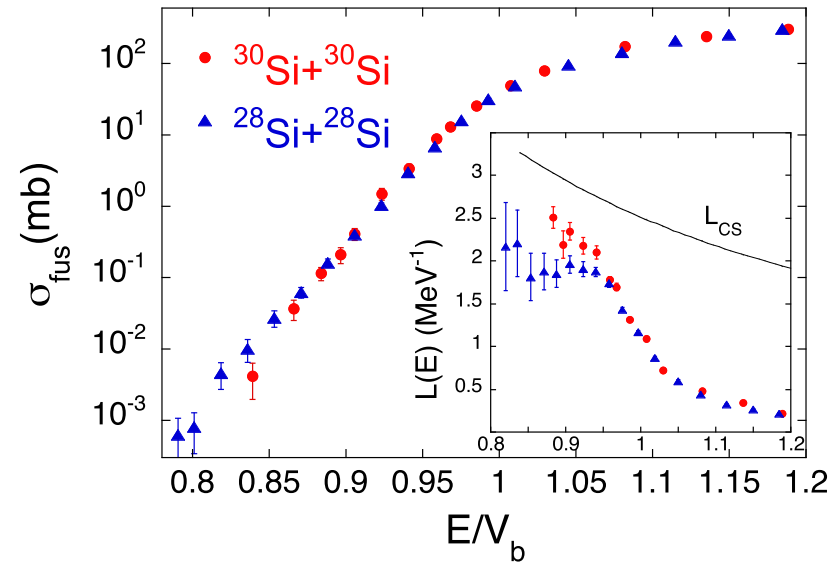

Fig. 27 Fusion excitation function of ${ }^{30} \mathrm{Si}+{ }^{30} \mathrm{Si}$ (see Ref. [160]), compared to the system ${ }^{28} \mathrm{Si}+{ }^{28} \mathrm{Si}$. The energy scale is normalized to the Akyüz-Winther Coulomb barrier [150]. The reported errors are purely statistical uncertainties. The insert shows the logarithmic derivatives (slopes) of the excitation functions for the two systems. $L(E)$ values were obtained with the two-points difference method. The values of $V_{b}$ are 28.10 and $28.80 \mathrm{MeV}$ for ${ }^{30} \mathrm{Si}+{ }^{30} \mathrm{Si}$ and ${ }^{28} \mathrm{Si}+{ }^{28} \mathrm{Si}$, respectivly

The logarithmic derivative, shown in the insert, displays an irregularity below the barrier, but no indication of an $S$-factor maximum appears. This behavior was tentatively attributed to the large oblate deformation of ${ }^{28} \mathrm{Si}$ because $\mathrm{CC}$ calculations largely underestimate the ${ }^{28} \mathrm{Si}+{ }^{28} \mathrm{Si}$ cross sections at low energies, unless a weak imaginary potential is applied, probably simulating the effect of deformation.

A more recent experiment was performed to clarify the underlying fusion dynamics by studying the case of ${ }^{30} \mathrm{Si}+{ }^{30}$ $\mathrm{Si} .{ }^{30} \mathrm{Si}$ has no permanent deformation and its low-energy excitations are vibrational in nature. The excitation function of ${ }^{30} \mathrm{Si}+{ }^{30} \mathrm{Si}$ was measured down to the level of a few $\mu \mathrm{b}$ [160]. It has a regular shape, at variance with the unusual trend of ${ }^{28} \mathrm{Si}+{ }^{28} \mathrm{Si}$. The extracted logarithmic derivative does not reach the $L_{c s}$ limit at low energies, as shown in Fig. 27, so that no maximum of the $S$ factor shows up.

$\mathrm{CC}$ calculations were performed including the low-lying $2^{+}$and $3^{-}$excitations. Using a Woods-Saxon potential the experimental cross sections at low energies are overpredicted, which is a clear sign of hindrance, while the calculations performed with a M3Y + repulsive potential nicely fit the data, without the need of an imaginary potential. Thus, the comparison with the results for ${ }^{28} \mathrm{Si}+{ }^{28} \mathrm{Si}$ strengthens the explanation of the oblate shape of ${ }^{28} \mathrm{Si}$ being the reason for the irregular behavior of that system.

The measured excitation function of ${ }^{28} \mathrm{Si}+{ }^{30} \mathrm{Si}[159$, 161] shows hindrance at a few $\mathrm{MeV}$ below the Coulomb barrier by comparing the data to $\mathrm{CC}$ calculations using a WS potential (see Fig. 28), whereas a nice fit is obtained when using the M3Y+rep potential, and including one- and twoneutron transfer channels in the coupling scheme, in addition to the low-lying surface excitations. The influence of transfer 


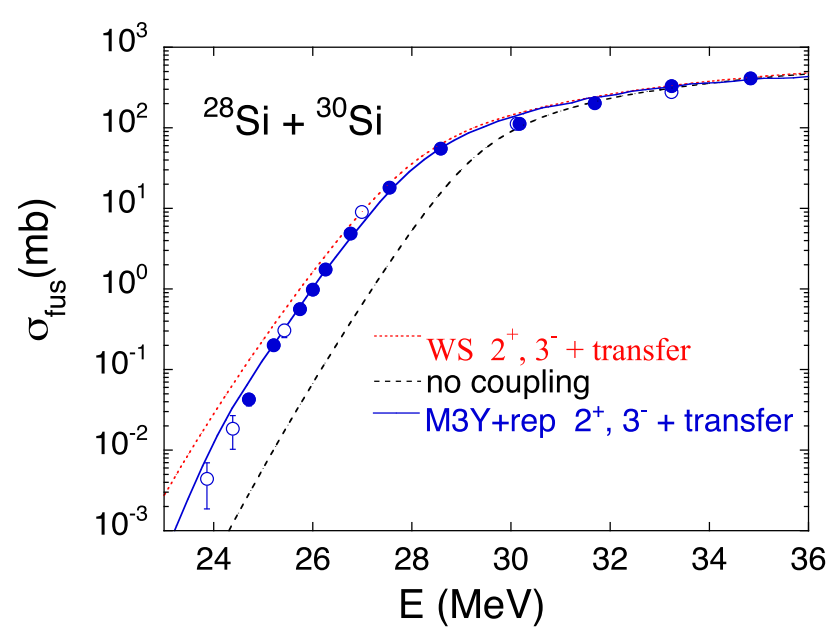

Fig. 28 Fusion excitation function of ${ }^{28} \mathrm{Si}+{ }^{30} \mathrm{Si}$ (see Ref. [159, 161]), compared to CC calculations employing the WS and the M3Y + repulsion potentials (see text). The filled and open symbols represent the cross sections measured in Ref. [161] and [159], respectively

is calculated to be small, as is the overall magnitude of the hindrance effect.

\subsection{Characteristics of the experimental $S(E)$}

Following the first observation of fusion hindrance in the ${ }^{60} \mathrm{Ni}+{ }^{89} \mathrm{Y}$ system by Jiang et al. [11], subsequent experi- ments have shown that fusion cross sections of other systems exhibit the hindrance effect at low energies as well, as shown by the existence of an $S$-factor maximum. It was also realized that many previously published measurements, performed down to deep sub-barrier energies, revealed the existence of fusion hindrance. In the following three figures, Figs. 29, 30, and 31 , several systems, with or without an observed $S(E)$ factor maximum, are discussed separately. In these figures and others shown later, data from earlier measurements are also included. It should be noted that most systems shown in these three figures have negative fusion $Q$ values, hence an $S$-factor maximum must appear at low energies.

Nine systems, where an $S$-factor maximum has been observed in the measured energy region, are shown in Fig. 29. The solid lines in the figure are smooth fits to the data. The systems ${ }^{90} \mathrm{Zr}+{ }^{92} \mathrm{Zr}$, ${ }^{89} \mathrm{Y}$ were measured at GSI in the 1980 's [33] down to very small cross sections ( $\simeq 120 \mathrm{nb}$ and $340 \mathrm{nb}$, respectively), below the onset of hindrance. Results for the system ${ }^{90} \mathrm{Zr}+{ }^{90} \mathrm{Zr}$ were obtained in the same experiments. However, as discussed in Sect. 2, contaminants from heavier $\mathrm{Zr}$-isotopes in the target can hamper the observation of an $S$-factor maximum in this case.

Four more examples for medium-mass systems are shown in Fig. 30. The corresponding standard CC calculations were performed using the Akyüz-Winther potential [150], including the lowest $2^{+}$and $3^{-}$collective modes. Although no $S$-factor maximum is apparent in any of these systems, we

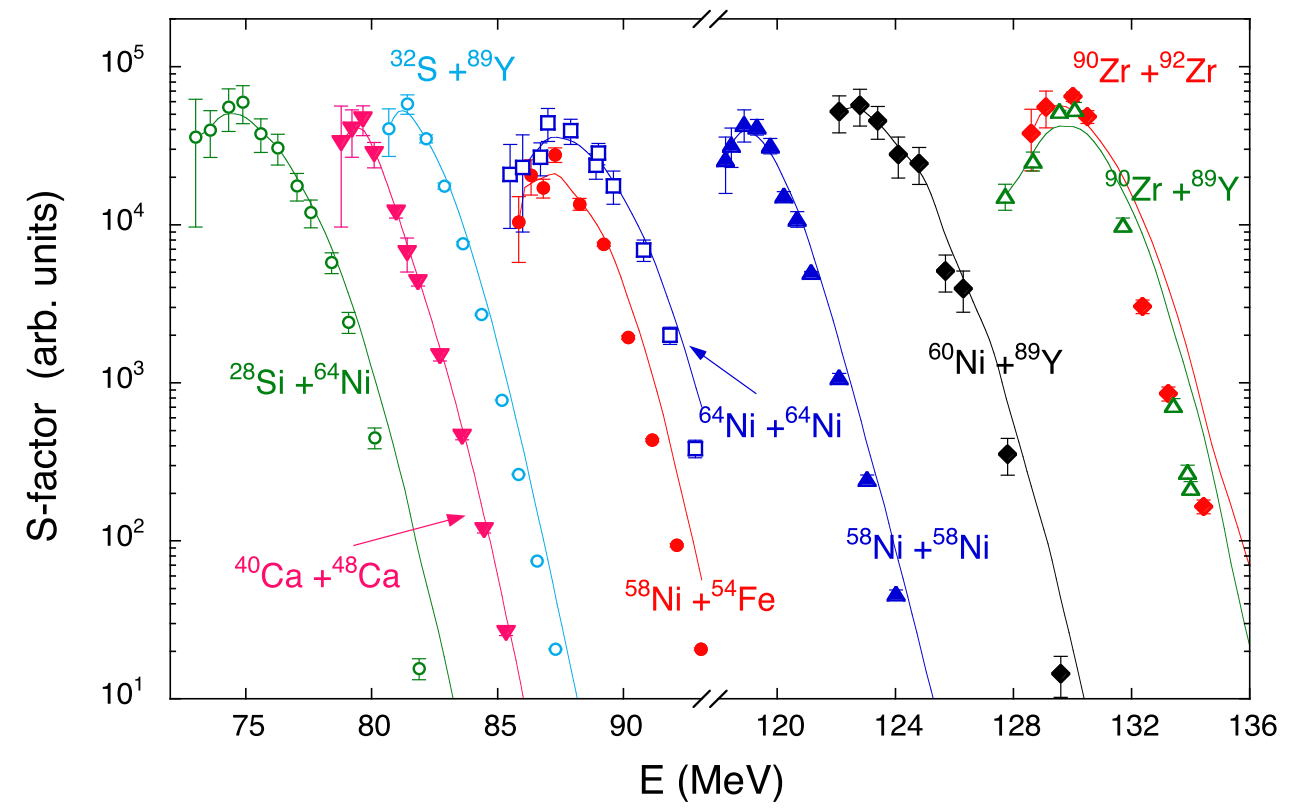

Fig. 29 Astrophysical $S$ factors of several systems where a maximum shows up. They were measured at ANL $\left({ }^{28} \mathrm{Si}+{ }^{64} \mathrm{Ni}[162],{ }^{64} \mathrm{Ni}+{ }^{64} \mathrm{Ni}\right.$ [12] and $\left.{ }^{60} \mathrm{Ni}+{ }^{89} \mathrm{Y}[11]\right), \mathrm{LNL}\left({ }^{40} \mathrm{Ca}+{ }^{48} \mathrm{Ca}[163]\right.$ and ${ }^{58} \mathrm{Ni}+{ }^{54} \mathrm{Fe}$ [46]), ANU-Canberra $\left({ }^{32} \mathrm{~S}+{ }^{89} \mathrm{Y}\right.$ [164]), MIT $\left({ }^{58} \mathrm{Ni}+{ }^{58} \mathrm{Ni}[16]\right)$ and GSI $\left({ }^{90} \mathrm{Zr}+{ }^{92} \mathrm{Zr},{ }^{89} \mathrm{Y}[33]\right)$. The energy scale has been shifted for some of the systems for graphical reasons, i.e. by $+25 \mathrm{MeV},+33 \mathrm{MeV},+33$ $\mathrm{MeV},-41 \mathrm{MeV},-41 \mathrm{MeV}$ and $+8 \mathrm{MeV}$ for ${ }^{58} \mathrm{Ni}+{ }^{58} \mathrm{Ni},{ }^{28} \mathrm{Si}+{ }^{64} \mathrm{Ni}$, ${ }^{40} \mathrm{Ca}+{ }^{48} \mathrm{Ca},{ }^{90} \mathrm{Zr}+{ }^{92} \mathrm{Zr},{ }^{90} \mathrm{Zr}+{ }^{89} \mathrm{Y}$ and ${ }^{32} \mathrm{~S}+{ }^{89} \mathrm{Y}$, respectively. Among these nine systems, only ${ }^{40} \mathrm{Ca}+{ }^{48} \mathrm{Ca}$, has a positive fusion $Q$ value 


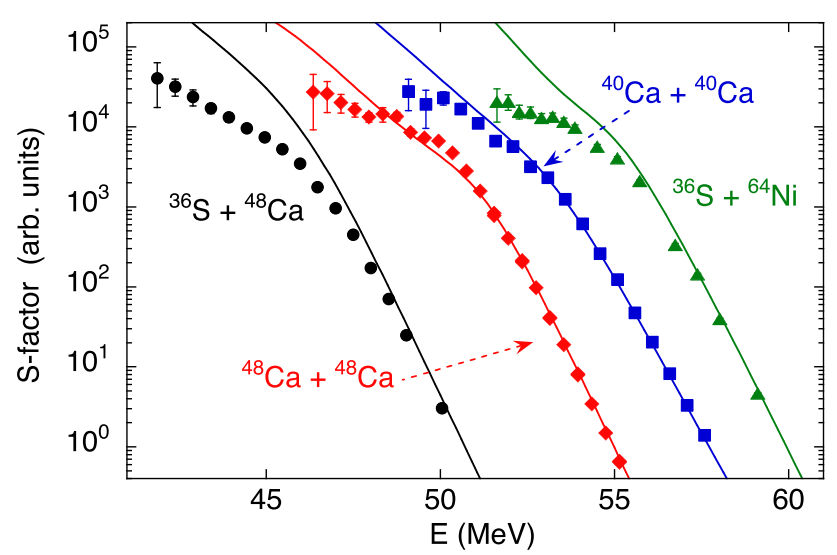

Fig. 30 Astrophysical $S$ factors for several systems where the fusion hindrance can be recognized by comparing the data with the results of standard CC calculations. The reported cases are ${ }^{36} \mathrm{~S}+{ }^{48} \mathrm{Ca}$ [165], ${ }^{48} \mathrm{Ca}+{ }^{48} \mathrm{Ca}[166],{ }^{40} \mathrm{Ca}+{ }^{40} \mathrm{Ca}$ [167] and ${ }^{36} \mathrm{~S}+{ }^{64} \mathrm{Ni}$ [168]. For ${ }^{36} \mathrm{~S}+{ }^{48} \mathrm{Ca}$ the energy scale has been shifted up by $5 \mathrm{MeV}$ for graphical convenience. Among these four systems, only ${ }^{36} \mathrm{~S}+{ }^{48} \mathrm{Ca}$, has a positive fusion $Q$ value

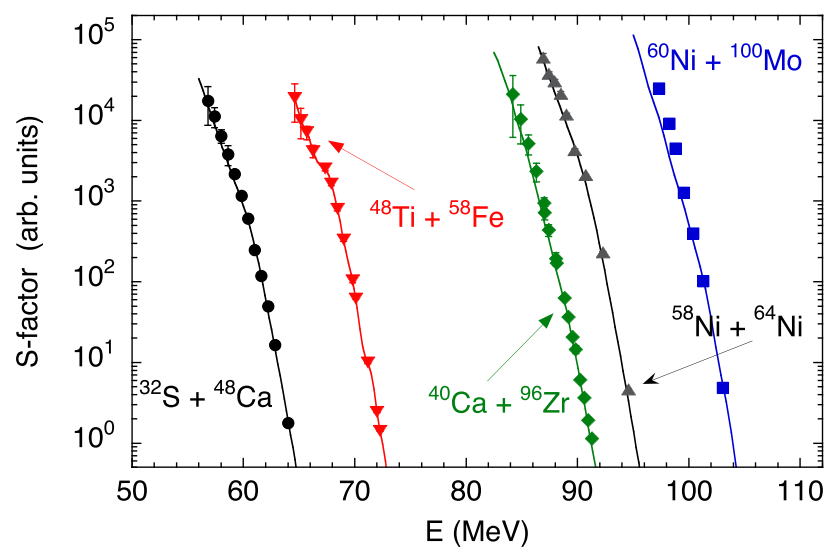

Fig. 31 Astrophysical $S$ factors for several systems where no maximum appears. The reported cases are ${ }^{32} \mathrm{~S}+{ }^{48} \mathrm{Ca}[169],{ }^{48} \mathrm{Ti}+{ }^{58} \mathrm{Fe}$ [144], ${ }^{40} \mathrm{Ca}+{ }^{96} \mathrm{Zr}[146,151],{ }^{58} \mathrm{Ni}+{ }^{64} \mathrm{Ni}[152]$ and ${ }^{60} \mathrm{Ni}+{ }^{100} \mathrm{Mo}$ [157]. For ${ }^{32} \mathrm{~S}+{ }^{48} \mathrm{Ca}$ and ${ }^{60} \mathrm{Ni}+{ }^{100} \mathrm{Mo}$ the energy scale has been shifted up by $20 \mathrm{MeV}$ and $-25 \mathrm{MeV}$, respectively, for graphical convenience. Among these five systems, only ${ }^{32} \mathrm{~S}+{ }^{48} \mathrm{Ca}$, has a positive fusion $Q$ value

see that the experimental $S$ factor is well below the CC calculations. One may therefore conclude that subbarrier fusion hindrance is present in these four systems. We thus expect that the $S$-factor maxima would appear if the measurements were extended to even lower energies.

However, there are other cases where even the comparison of the experimental $S$ factor with standard CC calculations does not give any indication of the fusion hindrance behavior. Figure 31 shows five examples of this situation which may be caused by the influence of nuclear structure and/or strong transfer couplings, that push down the hindrance threshold below the lowest measured energy. In particular, the case of ${ }^{40} \mathrm{Ca}+{ }^{96} \mathrm{Zr}[146,151]$ is a significant example of the effect of couplings to quasi-elastic transfer channels with $Q>0$, as discussed earlier in more detail. The recent data on ${ }^{58} \mathrm{Ni}+{ }^{64} \mathrm{Ni}[152]$ show a similar trend.

As discussed at the beginning of this section, another method to determine the location of an $S$-factor maximum uses the logarithmic derivative $L(E)$. An $S$-factor maximum appears at the crossing energy, $E_{S}$, of the experimental slope $L(E)$ with the curve given by the constant $S$ factor $L_{c s}(E)=\pi \eta(E) / E$.

Empirical expressions have been developed to extrapolate the excitation functions toward lower energies and to predict the energy, $E_{s}$, of the $S$-factor maximum for those systems which show only an indication of hindrance behavior in the measured energy range. These expressions reproduce the heavy-ion fusion excitation functions at low energies and can be used to extrapolate the data to even lower energies (see Refs. [170,171]) for systems, whose fusion $Q$ value are either positive or negative. These expressions are

$L(E)=A_{0}+\frac{B_{0}}{E^{3 / 2}}$, for $Q>0$,

or

$L(E)=A_{0}+\frac{B_{0}}{(E+Q)^{3 / 2}}$, for $Q<0$.

Here, $A_{0}$ and $B_{0}$ are parameters obtained from least-squares fits to the low-energy experimental slope $L(E)$. The corresponding expressions for the cross section at low energy are:

$$
\begin{aligned}
\sigma(E)= & \sigma_{s} \frac{E_{s}}{E} \exp \left(A_{0}\left(E-E_{s}\right)\right. \\
& \left.-\frac{2 B_{0}}{\sqrt{E_{s}}}\left[\sqrt{\frac{E_{s}}{E}}-1\right]\right), \text { when } Q>0,
\end{aligned}
$$

and

$$
\begin{aligned}
\sigma(E)= & \sigma_{s} \frac{E_{s}}{E} \exp \left(A_{0}\left(E-E_{s}\right)\right. \\
& \left.-\frac{2 B_{0}}{\sqrt{E_{s}+Q}}\left[\sqrt{\frac{E_{s}+Q}{E+Q}}-1\right]\right), \text { when } Q<0 .
\end{aligned}
$$

Here $\sigma_{s}$ is the cross section at the energy of the $S$ factor maximum, $E_{s}$, determined by the results of Eqs. (25) or (26). Thus, these extrapolation formulae have three free parameters, $A_{0}$, $B_{0}$, and $\sigma_{s}$. The energies, $E_{s}$, at the $S$-factor maximum for cases like those shown in Fig. 30 can be predicted.

The fusion $Q$ values of the systems shown in either Figs. 30 or 31 , are mostly negative, so that there must be an $S$-factor maximum at energies below the measured range. The extrapolation recipes, Eqs. 25-28 mentioned above, have been applied to these cases, and will be discussed in the following sections. 


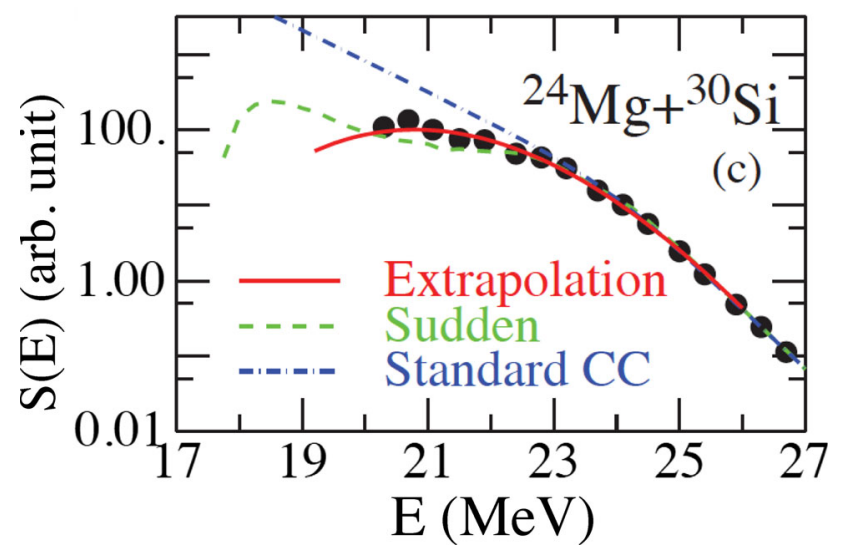

Fig. 32 Experimental $S(E)$ factor for the system, ${ }^{24} \mathrm{Mg}+{ }^{30} \mathrm{Si}$ compared to CC calculations using either a standard WS potential (blue dashed line) or the M3Y + repulsion potential (green dashed line). The extrapolation obtained from the empirical formula is also shown (red full line) [170]

\subsection{Toward astrophysical systems}

The fusion hindrance effect is inevitable for heavy systems with a positive energy threshold for compound nucleus formation $(Q<0)$. For cases of light or medium-mass systems, where the $Q$-value for fusion is positive, understanding the hindrance behavior will have far-reaching consequences in astrophysics where fusion of light systems determines latestage stellar evolution during the carbon and oxygen burning in heavy stars.

There are many studies of fusion cross sections at extreme low energies for ${ }^{12} \mathrm{C}+{ }^{12} \mathrm{C}$ (see [62,65] and Refs. therein) as well as for ${ }^{16} \mathrm{O}+{ }^{16} \mathrm{O}$. These cases will be discussed in detail in Sect. 5. These measurements are experimentally quite challenging and often result in large uncertainties and serious discrepancies among the different experiments in the low-energy range relevant for astrophysics. For that reason, similar investigations in slightly heavier systems can be very useful in order to validate the extrapolations towards these cases.

To this end, some cases with small negative or positive $Q$-values have been studied, including ${ }^{28} \mathrm{Si}+{ }^{64} \mathrm{Ni}$ [161], ${ }^{27} \mathrm{Al}+{ }^{45} \mathrm{Sc}[172],{ }^{40} \mathrm{Ca}+{ }^{48} \mathrm{Ca}$ [163], and ${ }^{36} \mathrm{~S}+{ }^{48} \mathrm{Ca}$ [165]. In these cases there is also evidence that the excitation functions drop faster than predicted by the CC calculations with a standard WS potential, indicating that fusion hindrance occurs.

Several systems with large positive $Q$-values have been studied recently. For ${ }^{24} \mathrm{Mg}+{ }^{30} \mathrm{Si}$, with $Q=+25.53 \mathrm{MeV}$, an $S$-factor maximum has been observed [173], as shown in Fig. 32. The blue dot-dashed curve is the result of CC calculations with a standard Woods-Saxon potential, which clearly overpredicts the experimental data at low energies. The green dashed line is a similar calculation using the shallow potential resulting from the M3Y + short-range repulsion [123,124].

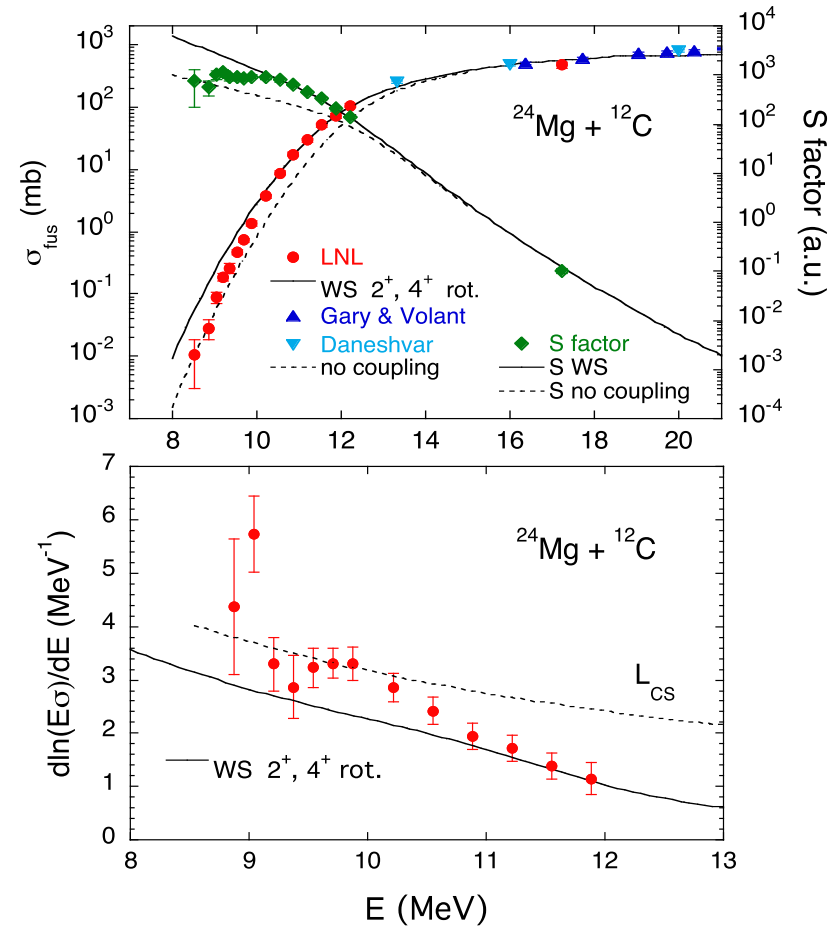

Fig. 33 (top) Excitation function and $S$ factor for the system ${ }^{12} \mathrm{C}+{ }^{24}$ $\mathrm{Mg}$, compared with standard CC calculations (see text for details). (bottom) Logarithmic derivative of the excitation function compared with the $L_{c s}$ value and with the CC calculations. Only statistical errors are reported in both panels. Figure adapted from Ref. [174]

The red full curve is calculated with the empirical extrapolation recipe of Ref. [170], which predicts an $S$-factor maximum, and reproduces the data better than the sudden approximation using the M3Y + repulsion potential.

These experiments have been recently extended to lighter systems. ${ }^{12} \mathrm{C}+{ }^{24} \mathrm{Mg}[174]$ and ${ }^{12} \mathrm{C}+{ }^{30} \mathrm{Si}[141]$ have a system parameter $\zeta$ very close to that of ${ }^{16} \mathrm{O}+{ }^{16} \mathrm{O}$ and ${ }^{12} \mathrm{C}+{ }^{12} \mathrm{C}$, and positive $Q$ values for fusion $(Q=+16.3 \mathrm{MeV}$ and +14.1 $\mathrm{MeV}$, respectively).

These two systems show interesting features of fusion hindrance which go beyond our previous knowledge. The measured cross sections for ${ }^{12} \mathrm{C}+{ }^{24} \mathrm{Mg}$ are shown in the upper panel of Fig. 33 together with the astrophysical $S$ factor. At the energy where the slope $L(E)$ (lower panel) reaches the $L_{c s}$ curve, the $S$ factor develops a maximum, which usually is taken as evidence for fusion hindrance. It is worthwhile to notice that the cross section at the hindrance threshold has a remarkably high value $(\sigma=1.6 \mathrm{mb})$. The reason why this happens, in this particular system, is presently unknown.

Evidence for the hindrance effect has also been observed for the system ${ }^{12} \mathrm{C}+{ }^{30} \mathrm{Si}$, whose fusion excitation function has been measured at LNL over a wide energy range. In Fig. 34, the excitation function is compared with the theoretical results of the adiabatic model [126-128] using the Yukawa-plus-exponential (YPE) potential (see lower panel 

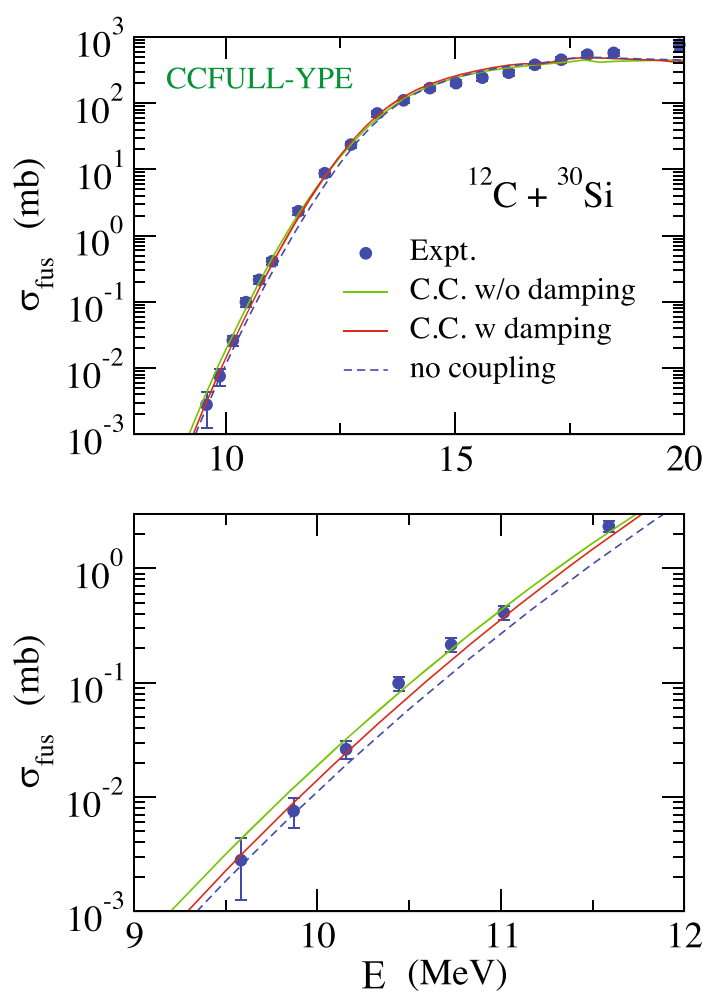

Fig. 34 The excitation function of ${ }^{12} \mathrm{C}+{ }^{30} \mathrm{Si}$ is compared to the results of various $\mathrm{CC}$ calculations employing the YPE potentials, with and without damping of the coupling strengths. The no-coupling limit is also reported. The lower panel is an expanded view of the low-energy range

of Fig. 34 for a detailed view). The result with and without quenching are both reported in the figure, and a slight preference for the calculation with quenching may be observed.

The $S$ factor appears to develop a maximum at lower energies shown in Fig. 35. This trend is supported by CC calculations performed by means of the adiabatic model (red curves), using both the WS and the YPE potentials [141]. However, the calculations are not able to reproduce the maximum around $10.5 \mathrm{MeV}$. In such a case, the energy threshold for the onset of hindrance is better identified by a comparison with the extrapolation curve (see the solid blue curve in Fig. 35). We point out that the cross section at the hindrance threshold for ${ }^{12} \mathrm{C}+{ }^{30} \mathrm{Si}$, is in the $10^{-2} \mathrm{mb}$ range, which is substantially smaller than that for the near-by system ${ }^{12} \mathrm{C}+{ }^{24} \mathrm{Mg}(1.6 \mathrm{mb})$.

In both systems, ${ }^{12} \mathrm{C}+{ }^{24} \mathrm{Mg}$ and ${ }^{12} \mathrm{C}+{ }^{30} \mathrm{Si}$, there are indications of an $S$-factor maximum with an energy $E_{S}$ that nicely fits in the systematics developed for medium-mass systems [175] (see Sect. 6).

One can also notice that the fusion cross sections of these systems would be fit, at the lowest measured energies, by CC calculations with coupling strengths completely damped. The cross sections at the lowest measured energies can, thus, be predicted by single-barrier penetration calculations (no

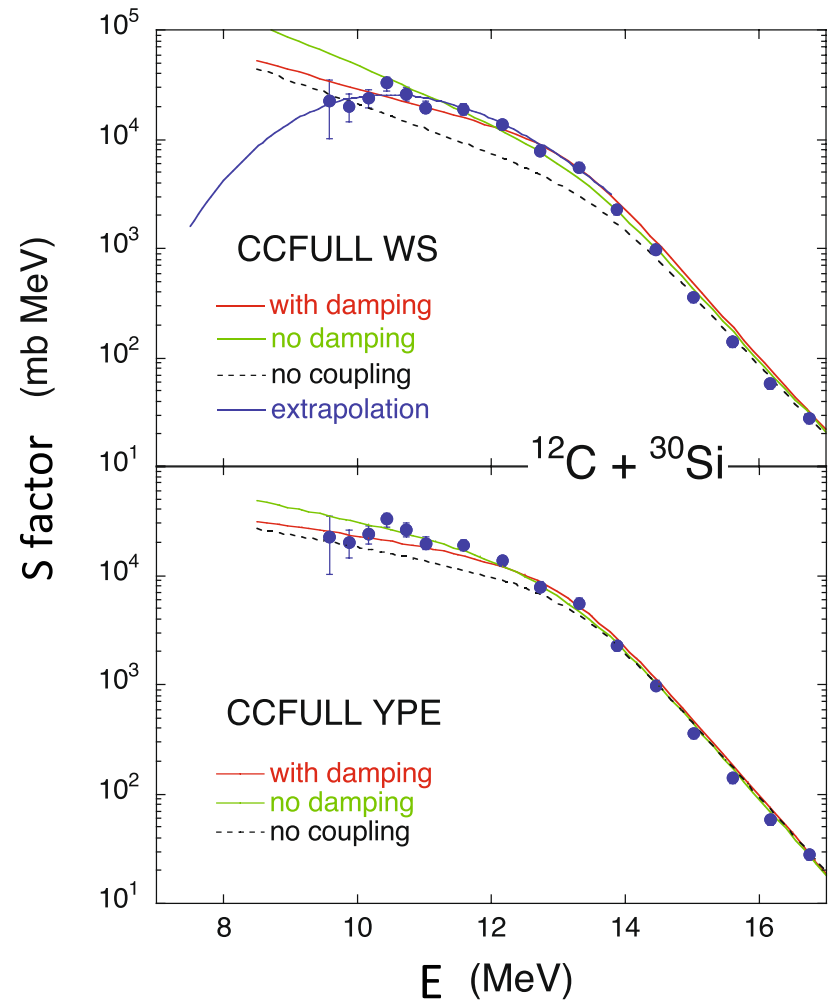

Fig. 35 Astrophysical $S$ factor for ${ }^{12} \mathrm{C}+{ }^{30} \mathrm{Si}$ in comparison with the $\mathrm{CC}$ calculations. A maximum of the $S$ factor vs. energy seems to develop around $10.5 \mathrm{MeV}$

couplings). This feature can be clearly seen for ${ }^{12} \mathrm{C}+{ }^{24} \mathrm{Mg}$ in the top panel of Fig. 33.

\section{Fusion Hindrance in light-mass systems}

The ${ }^{12} \mathrm{C}+{ }^{12} \mathrm{C},{ }^{12} \mathrm{C}+{ }^{16} \mathrm{O}$ and ${ }^{16} \mathrm{O}+{ }^{16} \mathrm{O}$ fusion reactions at low energies play an important role in the evolution of postmain sequence stars, in the ignition of type Ia supernovae, in accreting carbon-oxygen white dwarfs, and as a possible trigger in the ignition of superbursts on the surface of accreting neutron stars. Although these processes occur in explosive scenarios, the associated Gamow energies are still very low, resulting in extremely small cross sections, which in many cases are not yet experimentally accessible [89]. In order to obtain the astrophysical reaction rates, one has therefore to use phenomenological extrapolation methods.

It is therefore very important to understand the low-energy behavior of the astrophysical $S(E)$ factor for light fusion systems in order to address the question of a possible $S$ factor maximum in these fusion reactions, since this would strongly affect the astrophysical reaction rates [176].

Although fusion reactions in light-mass systems have positive $Q$-values and, thus, no maximum in the $S$ factor is required, it was shown in the previous section that an $S$ - 
factor maximum has been observed in systems as light as ${ }^{12} \mathrm{C}+{ }^{24} \mathrm{Mg}$. This maximum, however, was broad, in contrast to what was observed for heavier systems.

\subsection{General behavior of the $S(E)$ factor for light systems}

In this subsection we explore the evolution of the $S(E)$ factor as a function of the mass of the system. As discussed earlier, the $S$-factor maximum is observed where the logarithmic derivative $L(E)$ crosses the constant $S$-factor line, $L_{c S}(E)$. The system dependence of the logarithmic derivative $L(E)$ is shown in Fig. 36 covering fusion reactions from ${ }^{10} \mathrm{~B}+{ }^{10} \mathrm{~B}$ $(Q=+31.1 \mathrm{MeV})[177]$ to ${ }^{60} \mathrm{Ni}+{ }^{89} \mathrm{Y}(Q=-90.5 \mathrm{MeV})$ [11].

In this figure, experimental values of $L(E)$ are obtained numerically using the two-point (circles) or the three-point (stars) difference method to calculate the derivative and to compare to the constant $S$ factor, $L_{c s}(E)$, given by the blackdashed curves. The red curves are calculations from the extrapolation recipe using low-energy experimental data of $L(E)$ (See Eqs. 25 and 26.)

Several interesting features can be observed from Fig. 36. The crossing angle between $L(E)$ and $L_{c s}(E)$ is getting smaller going from heavier to lighter systems. For lighter systems, an $S$-factor maximum is therefore broader, and the energies at the maximum of the $S$ factor are less well-determined. This tendency has been studied in Ref. [158] and will be discussed further in Sect. 6.

The associated energies at the crossing points, $E_{S}$ which are about $123 \mathrm{MeV}$ for the ${ }^{60} \mathrm{Ni}+{ }^{89} \mathrm{Y}$ system, decrease to about $7 \mathrm{MeV}$ for the astrophysically important ${ }^{16} \mathrm{O}+{ }^{16} \mathrm{O}$ system. This has important consequences for the experimental techniques used in these measurements.

As discussed in Sect. 2, the techniques for fusion measurements in heavy systems involves in most cases the detection of the fusion-evaporation residues emitted at small scattering angles by using time-of-flight or electro-magnetic rigidity techniques. These techniques work well for heavier systems (e.g. ${ }^{60} \mathrm{Ni}+{ }^{89} \mathrm{Y}$ shown in Fig. 36, [11]) where the opening angle of the compound nuclei is $<3^{\circ}$, comparable to, for example, the acceptance angle of the FMA at ATLAS [38] or the electrostatic beam separator PISOLO at LNL [51]. For lighter systems, such as ${ }^{10} \mathrm{~B}+{ }^{10} \mathrm{~B}$ or ${ }^{16} \mathrm{O}+{ }^{16} \mathrm{O}$, the opening angle increases by more than a factor of 10 , and different detection techniques are therefore required. For example, for the study of fusion in the ${ }^{12} \mathrm{C}+{ }^{12} \mathrm{C}$ system, the detection of light evaporation particles (protons, neutrons and $\alpha$ 's) from the compound nuclei or $\gamma$ 's from the decay of the residual nuclei are used. The ${ }^{12} \mathrm{C}+{ }^{12} \mathrm{C}$ system will be discussed in more detail below. Experiments using different detection techniques are also important for getting a better understanding of the systematic uncertainties associated with the various experiments. More recently, dedicated setups to mea- sure particle- $\gamma$ coincidences have also been used to measure fusion cross sections at very low energies. Advantages and disadvantages of all these techniques including background reactions from contaminants in the carbon targets are discussed in Sect. 2 and in the respective publications [37, 56, 58, 62, 65, 178, 179].

Another feature that can be observed from Fig. 36 is the occurrence of oscillations in some of the systems. It is well known that oscillations or resonances have been seen in the excitation functions of ${ }^{12} \mathrm{C}+{ }^{12} \mathrm{C},{ }^{12} \mathrm{C}+{ }^{16} \mathrm{O}$ and other fusion systems, whose reaction participants are $\alpha$-cluster nuclei, as early as in the 1960's. While most of the logarithmic derivatives shown in Fig. 36 exhibit a smooth increase of $L(E)$ towards lower energies, some systems, e.g. ${ }^{12} \mathrm{C}+{ }^{24} \mathrm{Mg}$ and ${ }^{12} \mathrm{C}+{ }^{30} \mathrm{Si}$, show oscillations in the logarithmic derivative. These oscillations make it difficult to identify the $S$-factor maximum in these systems.

It should be mentioned, that at energies above the Coulomb barrier, $L(E)$ is always lower than the $L_{c s}(E)$. Both curves increase when the energy decreases. A crossover may appear only when the logarithmic derivative, $L(E)$ is steeper than the $L_{c s}(E)$ curve. For fusion of ${ }^{10} \mathrm{~B}+{ }^{10} \mathrm{~B}$, the experimental $L(E)$ does not cross or touch the $L_{c s}(E)$ curve within the measured energy range.

\subsection{The $S$-factor for the ${ }^{16} \mathrm{O}+{ }^{16} \mathrm{O}$ system}

Fusion in the ${ }^{16} \mathrm{O}+{ }^{16} \mathrm{O}$ system has been measured in four different experiments in the 1970s using both gas as well as solid targets by detecting either $\gamma$ rays or light charged particles from the decay of the compound nuclei and covering the energies from $\sim 7$ to $11 \mathrm{MeV}$, [180-184]. The lowest cross sections measured in these experiments are $\sim 5 \mu \mathrm{b}$. In addition, two more experiments using solid targets and $\gamma$ detection techniques covering energies down to $8 \mathrm{MeV}$ [185] and 8.3 MeV [186] have been published. The results, converted into astrophysical $S$ factors, are shown in Fig. 37, along with an optical model calculation shown by the blue dashed line.

While there is a reasonable agreement among the different experiments at the higher energies, the cross sections at $E \sim$ $7 \mathrm{MeV}$ differ by a factor of about four. Analyzing each data set separately, one observes that the majority of the data show a maximum in the $S(E)$. However, the maximum does not occur at the same energy in the different measurements. As a result, showing these experimental data together, as done in Fig. 37, the $S$-factor maximum becomes poorly defined.

The black-dashed curve in Fig. 37 is the result of Fowler's $S$-factor parameterization ([89]) given by

$S(E)=S_{0} \frac{\exp (-\alpha E)}{\exp \left(-\gamma E^{m}\right)+b \exp (\beta E)}$, 

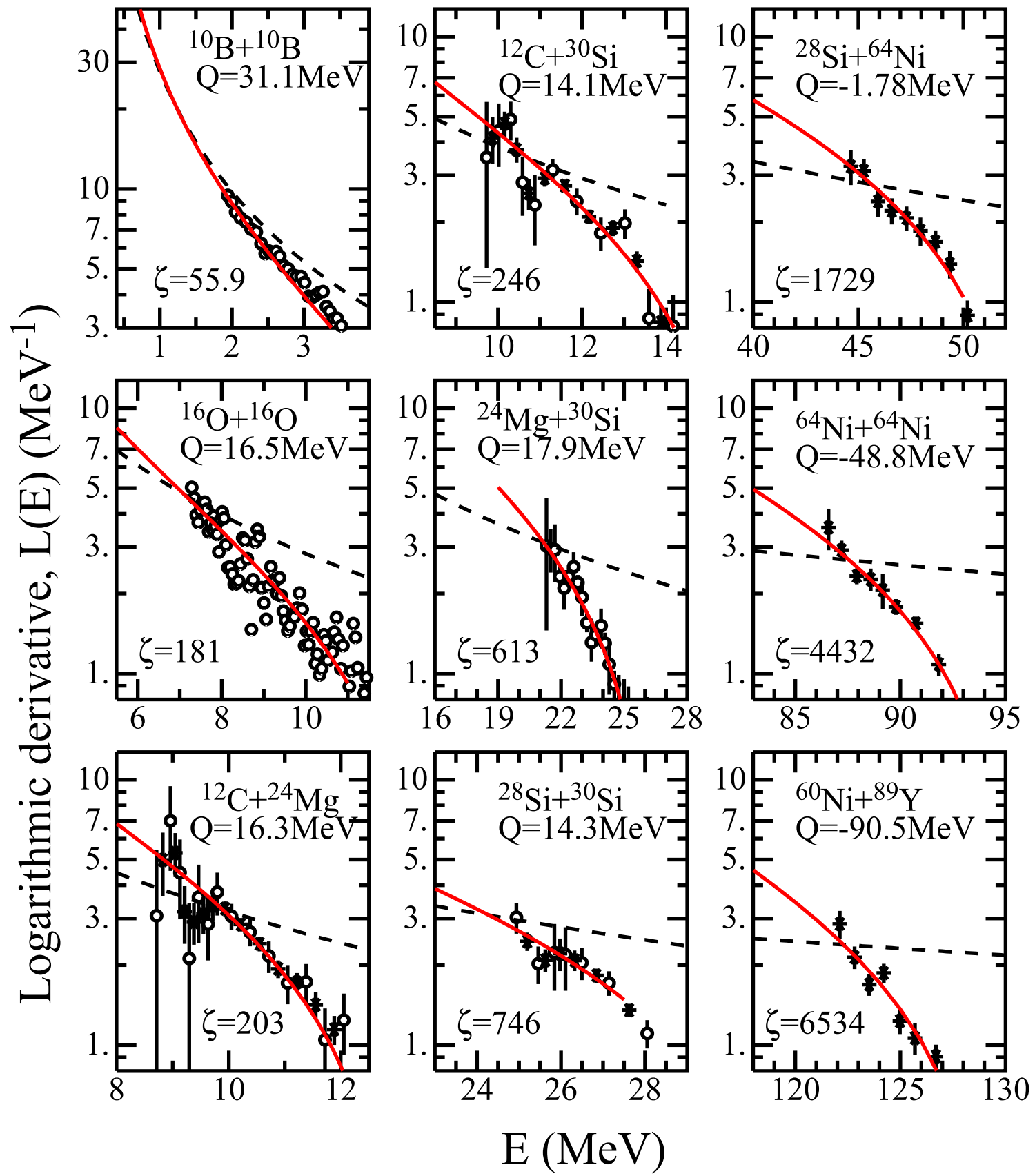

Fig. 36 Logarithmic derivatives $L(E)$ for nine systems ranging from ${ }^{10} \mathrm{~B}+{ }^{10} \mathrm{~B}$ to ${ }^{60} \mathrm{Ni}+{ }^{89} \mathrm{Y}$. Symbols are $L(E)$, derived from experimental cross sections, while the black dashed curves represent the constant$S$-factor function $L_{c s}(E)$. The red curves are calculations from the extrapolation recipe by using low-energy experimental data of $L(E)$.
For ${ }^{10} \mathrm{~B}+{ }^{10} \mathrm{~B}$ and ${ }^{16} \mathrm{O}+{ }^{16} \mathrm{O}, L(E)$ values are obtained with the fivepoints method and uncertainties are not shown. For ${ }^{16} \mathrm{O}+{ }^{16} \mathrm{O}$ only Spinka's data are given (see Fig. 38 for details). For the other systems, open circles and stars are obtained with two- and three-points difference methods, respectively. See text for details where $S_{0}, \alpha, \beta, \gamma, b$ and $m$ are six adjustable fit parameters. The red curve is obtained from the hindrance parameterization obtained in Ref. [170] with three adjustable parameters, $\sigma_{s}, A_{0}, B_{0}$. Both extrapolations predict a nearly constant $S$ factor at energies below $8 \mathrm{MeV}$, in agreement with the data, but a different trend for energies below $\sim 7 \mathrm{MeV}$, where no experimental data exist.

As discussed earlier, another approach to locate the maximum of the $S$ factor is by determining the crossing point of the logarithmic derivative with the constant $S$ factor curve $L_{c s}$. The results of this approach for the ${ }^{16} \mathrm{O}+{ }^{16} \mathrm{O}$ system 


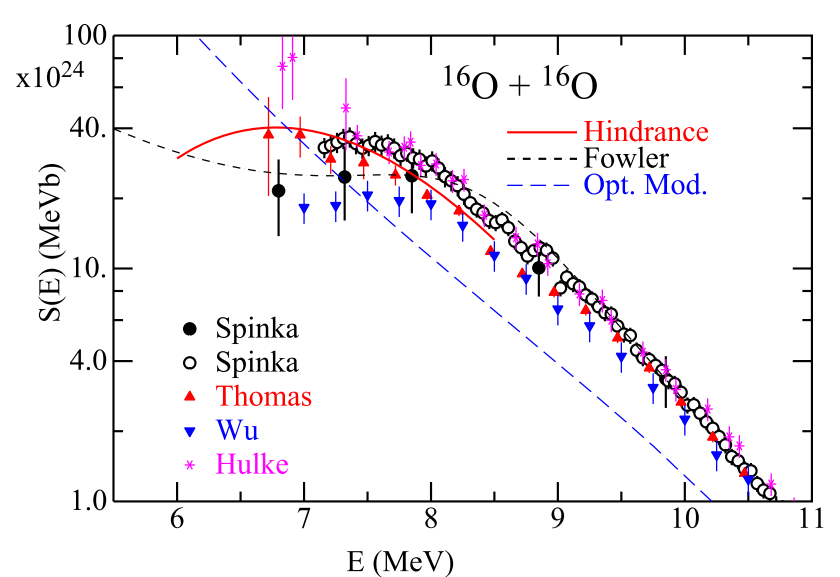

Fig. $37 S(E)$ factors for the system ${ }^{16} \mathrm{O}+{ }^{16} \mathrm{O}$ from different experiments. The red curve, obtained from the hindrance extrapolation model predicts a maximum in the $S$-factor curve, while Fowler's parameterization result predicts a continuous rise at low energies

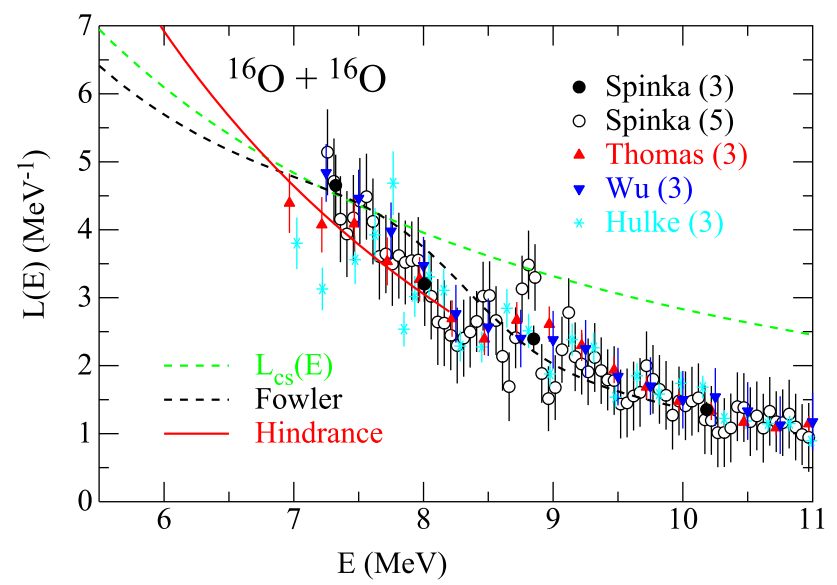

Fig. 38 Logarithmic derivative $L(E)$ for the system ${ }^{16} \mathrm{O}+{ }^{16} \mathrm{O}$. The crossing point of the $L(E)$ and the constant $S$ factor function $L_{c s}(E)$ determines the energy location of the $S$-factor maximum. The red curve is obtained from the least-squares fit (see text for details). The $L(S)$ values represented by black solid, black open, red triangle, blue triangle and star were obtained with 3-, 5-, 3-. 3-, and 3-points difference methods, respectively

are shown in Fig. 38. The symbols and curves are the same as the ones used in Fig. 37. Although the $S$-factor data for ${ }^{16} \mathrm{O}+{ }^{16} \mathrm{O}$ differ at the lowest energies by factor of four, the derivative data show a much better agreement among the various experiments, since the logarithmic derivative $L(E)$ is not sensitive to the absolute values of the excitation functions. The crossing between $L(E)$ and $L_{c s}(E)$ (green dashed line) occurs around $7 \pm 0.5 \mathrm{MeV}$. The narrow oscillating structure at $E=8.8 \mathrm{MeV}$ is only observed in the data by Spinka et al. which are the only measurements that have been taken at small enough energy steps to observe this feature. The structure observed in the data of Hulke et al. at $7.75 \mathrm{MeV}$, the only experiment for the ${ }^{16} \mathrm{O}+{ }^{16} \mathrm{O}$ system using a gas

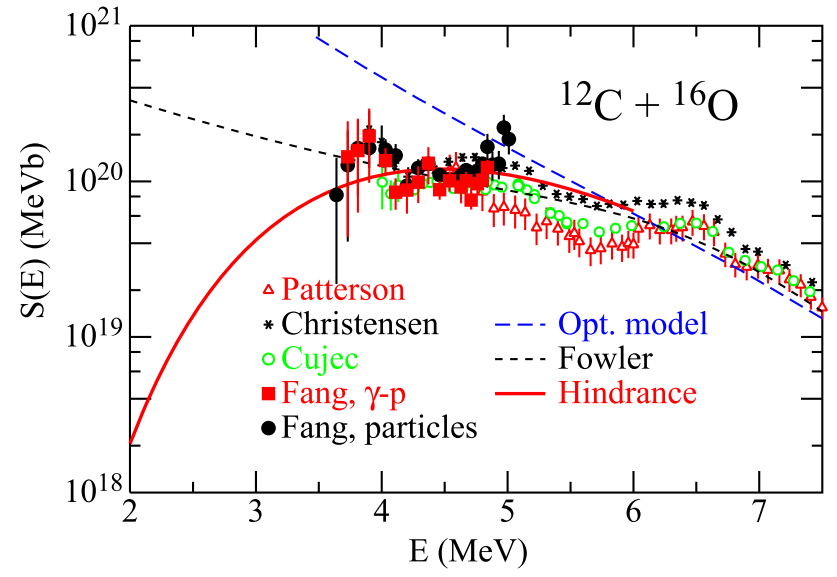

Fig. $39 S(E)$ factor for the system ${ }^{12} \mathrm{C}+{ }^{16} \mathrm{O}$ as measured in different experiments. The results from the recent measurements by Fang et al. are shown by the larger symbols. The black dashed line is the prediction by Fowler et al. [89]. The prediction from the hindrance model is given by the red solid line

target and $\gamma$ detection, has not been reproduced in the other measurements.

No new measurements for this system have been published since the analysis of these data with the hindrance model in 2007 [170]. Since an $S$-factor maximum has been seen in most measurements, we conclude that, the ${ }^{16} \mathrm{O}+{ }^{16} \mathrm{O}$ data have shown an evidence of a maximum, though more data are needed to confirm this conclusion.

\subsection{The $S(E)$ factor for the ${ }^{12} \mathrm{C}+{ }^{16} \mathrm{O}$ system}

Similar to the ${ }^{16} \mathrm{O}+{ }^{16} \mathrm{O}$ system, most of the experiments for ${ }^{12} \mathrm{C}+{ }^{16} \mathrm{O}$ were performed in the 1970 's $[84,187,188]$, using ${ }^{16} \mathrm{O}$ beams and natural carbon targets and detecting the decay of the compound nuclei by either particle or $\gamma$-decays. More recently, Fang et al. [61] remeasured this reaction with both singles and particle- $\gamma$ coincidence techniques. The coincidence method, first used at ATLAS for measuring the fusion of ${ }^{12} \mathrm{C}+{ }^{12} \mathrm{C}$ at low energies [55,56], is crucial to reduce background effects which hamper measurements of fusion cross sections for light-mass systems at very low energies. The lowest cross section measured for this system was $1.1 \pm 0.8$ nb, i.e. a factor of $10^{3}$ lower than for the ${ }^{16} \mathrm{O}+{ }^{16} \mathrm{O}$ system.

The experimental results of the $S(E)$ factor and the logarithmic derivative $L(E)$ are shown in Figs. 39 and 40, respectively. In these figures, the recent data by Fang et al. which extend to an energy of about $3.6 \mathrm{MeV}$, are shown by larger symbols.

A distinct difference between the ${ }^{16} \mathrm{O}+{ }^{16} \mathrm{O}$ and ${ }^{12} \mathrm{C}+{ }^{16}$ $\mathrm{O}$ systems are the oscillations, observed in the excitation functions of the ${ }^{12} \mathrm{C}+{ }^{16} \mathrm{O}$ systems. We note that the fusion oscillations in this system at energies above the Coulomb barrier have been attributed to elastic alpha transfer channel. 


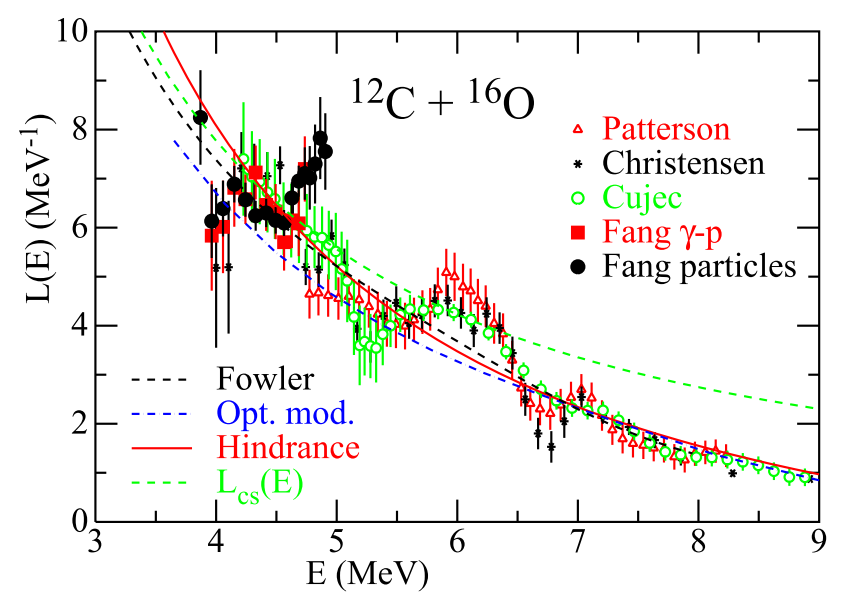

Fig. 40 Logarithmic derivative $L(E)$ for the system ${ }^{12} \mathrm{C}+{ }^{16} \mathrm{O}$ from different experiments. The values of $L_{s}$ given by symbols of red triangle, star, open circle, square and solid circle were obtained with 8-, 5-, 4-. 6-, and 6-points difference methods, respectively. The crossing point of $L(E)$ and the constant $S$ factor function $L_{c s}(E)$ (dashed green line) determines the energy of the $S$-factor maximum. The predictions from Fowler's parameterization and from the hindrance model are given by the black-dashed and red-solid lines, respectively

These oscillations make the extraction of a possible $S$ factor maximum very difficult as can be seen from Fig. 39. The experimental data averaged over the oscillations are in agreement with both the Fowler and the hindrance model. Differences between the two extrapolations are expected for energies below $\sim 4.5 \mathrm{MeV}$, where, at this time, data with small enough uncertainties are not available. Cross section measurements in the nb and sub-nb range are non-trivial and subject to a variety of systematic uncertainties. Different detection techniques are needed in order to get a better understanding of the underlying systematics uncertainties.

In Ref. [170], the three earlier data sets were analysed with the hindrance model using the $L(E)$ data. Calculations yielded an $S$-factor maximum at $E_{s}=4.54 \mathrm{MeV}$. In Figs. 39 and 40 we show the new fit hindrance results including the recently measured data by the red curves, which give an $S$ factor maximum at a slightly lower energy of $E_{s}=4.41$ $\mathrm{MeV}$. Other calculations, such as Optical Model calculations or Fowler's extrapolation recipe, predict a continuous increase of the $S$ factor toward even lower energies. Their $L(E)$ curves do not cross the constant $S$ factor curve $L_{c s}(E)$. It should be noted that at very low energies, the $L(E)$ function of Fowler's expression becomes (see [89])

$L_{\text {Fowler }}=L_{c s}(E)-\alpha$,

where $\alpha=0.64 \mathrm{MeV}^{-1}$ for this system.

Thus, at extreme low energies, $L_{F o w l e r}(E)$ will be parallel to $L_{s}(E)$ but always $0.64 \mathrm{MeV}^{-1}$ lower than the $L_{c s}(E)$, i.e. that it will never cross the $L_{c s}(E)$ curve.
In Ref. [61], it was hypothesized that there is a decrease in the $S(E)$ factor towards even lower energies, which may be due to hindrance in the fusion probability. However, because of the resonance-like structures, this conclusion may be premature, but the empirical extrapolation recipe (with expression $L(E)=A_{0}+B_{0} / E^{3 / 2}$ and the corresponding excitation function formula) works well at low energies, at least for systems as light as ${ }^{12} \mathrm{C}+{ }^{16} \mathrm{O}$.

\subsection{The $\mathrm{S}(\mathrm{E})$ factor for the ${ }^{12} \mathrm{C}+{ }^{14} \mathrm{~N}$ system.}

The fusion cross sections for the system ${ }^{12} \mathrm{C}+{ }^{14} \mathrm{~N}$, measured in the 1970s ([94]), by detecting the $\gamma$ decays of the residual nuclei, have already been presented in Fig. 12 (see Sect. 2), where they were found to be well described by the potential model. Together with the neighboring ${ }^{16} \mathrm{O}+{ }^{14} \mathrm{~N}$ system, mentioned later in Sect. 6, these are the only two fusion reactions discussed in this review leading to an oddodd compound nucleus. The influence of this nuclear structure effect will be discussed later in this section. The cross sections (open circles) for the ${ }^{12} \mathrm{C}+{ }^{14} \mathrm{~N}$ systems together with the corresponding $S$-factors ( black stars) are shown in Fig. 41. There may be a hint of a possible $S$-factor maximum at $\sim 3.4 \mathrm{MeV}$, but it would be necessary to measure the fusion cross sections for this reaction to even lower energies in order confirm this hypothesis. The black dashed curve in Fig. 41 is taken from the potential model which reproduces the experimental cross sections quite well, but does not yield a maximum in the $S$-factor. The red curve originates from the hindrance model which gives a maximum of the $S$ factor close to the lowest energies measured in this experiment.

The logarithmic derivative is presented in Fig. 42, with open circles and stars referring to $L(E)$ values obtained with the two- and three-point difference methods, respectively. The green curve gives the constant $S$-factor function $L_{c s}(E)$ while the red curve is a fit to the experimental data with the expression $A_{0}+B_{0} / E^{3 / 2}$. Although the uncertainties for the experimental values of $L(E)$ are large, the results indicate that they already touch or cross the $L_{c s}$ at the lowest energies.

\subsection{The $\mathrm{S}(\mathrm{E})$ factor for the ${ }^{12} \mathrm{C}+{ }^{13} \mathrm{C}$ system.}

Similar to the other systems discussed in the previous subsections, fusion experiments on ${ }^{12} \mathrm{C}+{ }^{13} \mathrm{C}$ cover a time range of more than 40 years starting with the first experiment in 1976. The early experiments covered the energy range down to about $3.2 \mathrm{MeV}$ and were all done using thin carbon targets and detecting $\gamma$-rays or light particles from the decay of the residual nuclei $[90,189,190]$. The two most recent experiments, $[68,191]$ were carried out after 2012 at the University of Notre Dame and at the Horia Hulubei National Institute, Romania. These measurements used the thick-target technique detecting the radioactive ${ }^{24} \mathrm{Na}\left(\mathrm{T}_{1 / 2}=14.95 \mathrm{~h}\right)$. From 


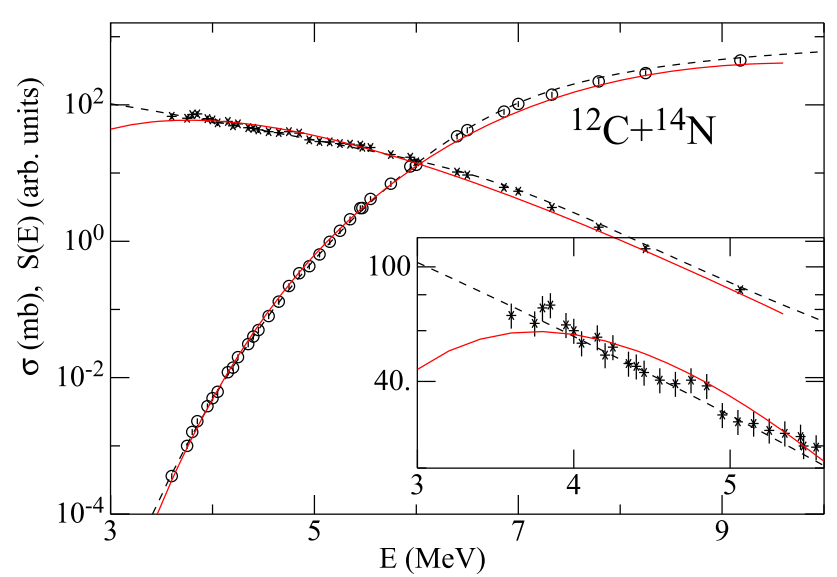

Fig. 41 Cross sections (open circles) and S-factors $S(E)$ (stars) for fusion in the system ${ }^{12} \mathrm{C}+{ }^{14} \mathrm{~N}$ from Ref. [94]. The black dashed curve uses the potential model shown in Fig. 12, while the red curve originates from the hindrance model ([170])

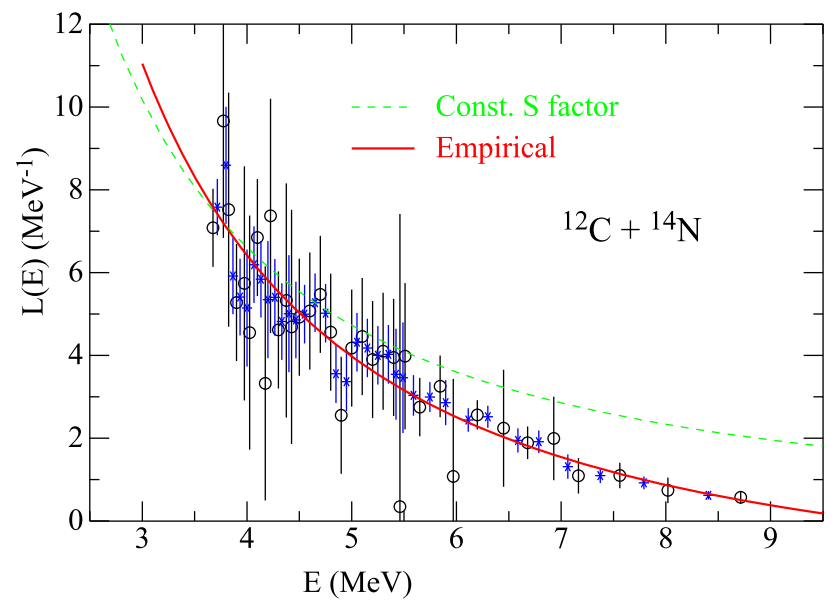

Fig. 42 Logarithmic derivatives $L(E)$ for the system ${ }^{12} \mathrm{C}+{ }^{14} \mathrm{~N}$ from Ref. [94] using the three-point method. The green dashed curve is the constant $\mathrm{S}$-factor function $\mathrm{L}_{c S}(E)$, while the red curve is a fit with the function $A_{0}+B_{0} / E^{3 / 2}$. The $L(E)$ values given by circles and stars were obtained with the two- or three-points difference methods, respectively

the yields, the total fusion cross sections were then extrapolated using statistical model calculations. In the second experiment, the radioactivity was measured in an underground counting laboratory. At an energy $E=2.323 \mathrm{MeV}$ the total fusion cross section obtained was $0.9 \pm 0.3 \mathrm{nb}$.

The results from these experiments, plotted as $S(E)$ factor or as logarithmic derivatives $L(E)$ are shown in Figs. 43 and 44 , respectively. The black dashed and red solid curves represent fits to the data available in 2007 using Fowler's parameterization and the hindrance model, respectively (see Ref. [170]). Similar to the ${ }^{12} \mathrm{C}+{ }^{14} \mathrm{~N}$ system, the experimental data show only very small fluctuations. The results from the recent experiments $[68,191]$, both done with the thick target technique, are in disagreement with the hindrance model,

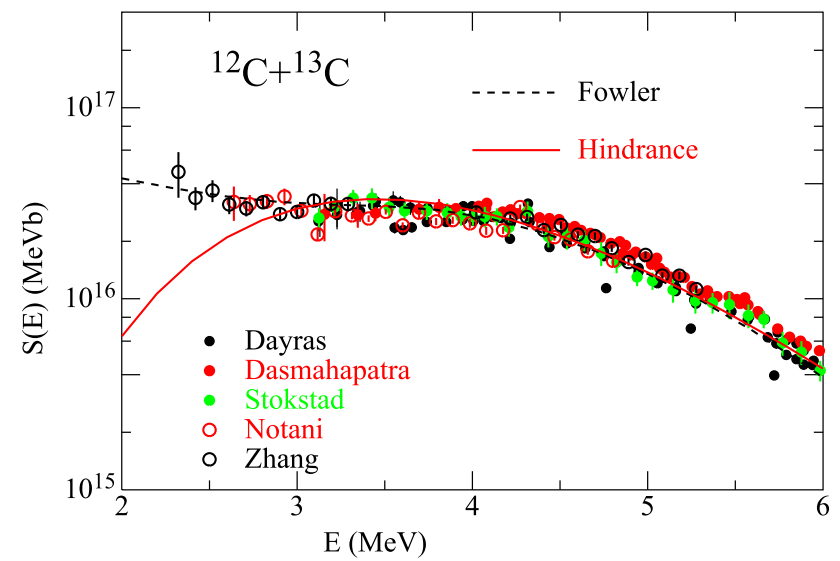

Fig. $43 \mathrm{~S}$-factor $S(E)$ for fusion in the system ${ }^{12} \mathrm{C}+{ }^{13} \mathrm{C}$ measured in different experiments. The recent thick target measurements are shown by larger symbols (Notani [191] and Zhang [68]). The predictions from Fowler's parameterization and from the hindrance model, obtained in 2007 [170] are given by the black dashed red solid lines, respectively

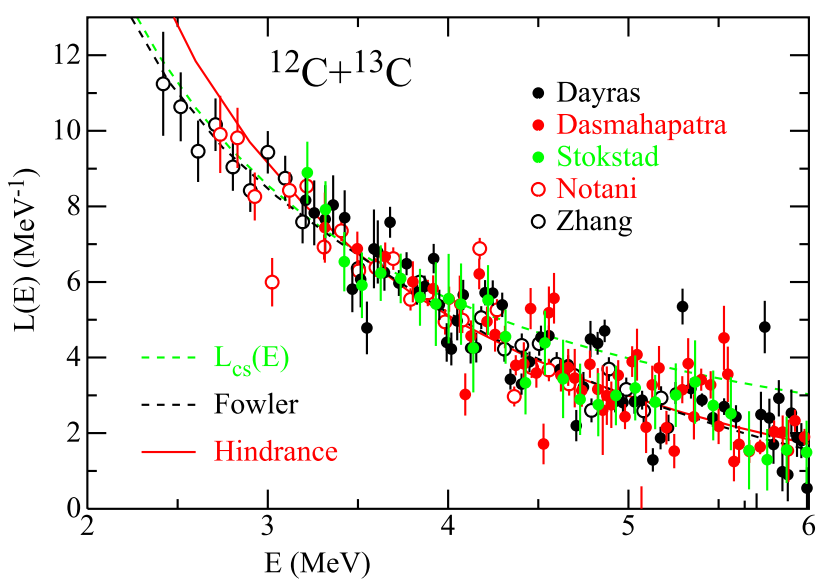

Fig. 44 Logarithmic derivative $L(E)$ for the system ${ }^{12} \mathrm{C}+{ }^{13} \mathrm{C}$ as measured in different experiments. The $L(E)$ values represented by black dashed, red solid, green solid, red open and black open symbols were obtained with 5-, 3-, 3-. 3-, and 4-points difference methods, respectively. The symbols and the lines are the same as in Fig. 43

but follow the Fowler parameterization as shown in Fig. 43. More experiments using different detection techniques would be helpful for this reaction. These data do not show evidence for an $S$-factor maximum for the ${ }^{12} \mathrm{C}+{ }^{13} \mathrm{C}$ system.

\subsection{Fusion in the ${ }^{12} \mathrm{C}+{ }^{12} \mathrm{C}$ system}

Carbon, after hydrogen, helium and ${ }^{16} \mathrm{O}$, the fourth most abundant element in the universe, plays an important role in the stellar nucleosynthesis. Fusion between two ${ }^{12} \mathrm{C}$ nuclei can occur in quiescent carbon burning in the core of massive stars and explosively in the ignition phase of type Ia supernovae, or in so-called superbursts, which take place in the surface region of neutron stars. The important energy range in these astrophysical scenarios is $1-3 \mathrm{MeV}$ in the center 
of mass, a region that has only recently become accessible for study in the laboratory. For that reason many astrophysics calculations still rely on extrapolations from fusion cross sections made at higher energies into the $1-3 \mathrm{MeV}$ range.

In Ref. [170], the earlier fusion data for ${ }^{12} \mathrm{C}+{ }^{12} \mathrm{C}$ (Ref. [192-194]) have been analyzed within the hindrance model. Since the fusion $Q$-value for this system is positive, no maximum of the $S$ factor must occur at low energies. By using the low energy $L(E)$ fitting recipe, an $S$-factor maximum at $E_{S}$ $=3.68 \mathrm{MeV}$ was predicted, at variance with the predictions from other calculations (e.g. Fowler et al.), which all predict a rise of the $S(E)$ factor. Gasques et al. [176] found that this difference has strong consequences for stellar burning and nucleosynthesis .

Several new measurements have recently pushed the energy limits to lower and lower values. In addition to early experiments by Kettner et al. [197] and Becker et al. [198], several new measurements by Barron-Palos et al. [195], Aguilera et al. [196], Spillane et al. [37] and Zickefoose et al. $[58,178]$, have measured the excitation function of ${ }^{12} \mathrm{C}+{ }^{12} \mathrm{C}$ by detecting either charged particles or $\gamma$-rays. Various experimental difficulties in these types of measurements were discovered and a cross section of about $35 \mathrm{pb}$ at energies of $2.15 \mathrm{MeV}$ has been extracted from Zickefoose's experiment [58]. In order to reduce the serious backgrounds encountered in singles experiments (measuring particles or $\gamma$ 's), the particle- $\gamma$ coincidence technique was applied for the first time to the ${ }^{12} \mathrm{C}+{ }^{12} \mathrm{C}$ system in 2012 [55]. So far three measurements with this technique have been performed at ATLAS [56], at the Androméde accelerator of the Institut de Physique Nucléaire, Orsay [65], and at the University of Notre Dame [62].

The results from direct measurements of ${ }^{12} \mathrm{C}+{ }^{12} \mathrm{C}$ fusion reactions in the energy range between 2.2 and $5.5 \mathrm{MeV}$ performed since 2002 and converted into $S$ factors are shown by various symbols in Fig. 45.

The $S$ factors from the most recent particle- $\gamma$ coincidence experiments are shown by larger symbols. The results from singles experiments, detecting either charged particles or $\gamma$ 's are shown by smaller circles. Extrapolations from Fowler et al. [89] (black-dashed curve) and the hindrance model [170], (red curve) are shown for comparison with the measurements.

While measurements using the particle $-\gamma$ coincidence technique are less affected by background reactions, the lower detection efficiency leads to longer running times and, thus, below $E \sim 2.6 \mathrm{MeV}$ only upper limits of the fusion cross sections have been obtained so far, namely $\sigma<580$ $\mathrm{pb}$ [65] at $2.16 \mathrm{MeV}$ and $\sigma<240 \mathrm{pb}$ [62] at $2.2 \mathrm{MeV}$. Singles measurements have been performed in smaller energy steps (see Fig. 45) but with limited accuracy due to the uncertainties associated with the thick target technique (see Refs. $[37,58])$.

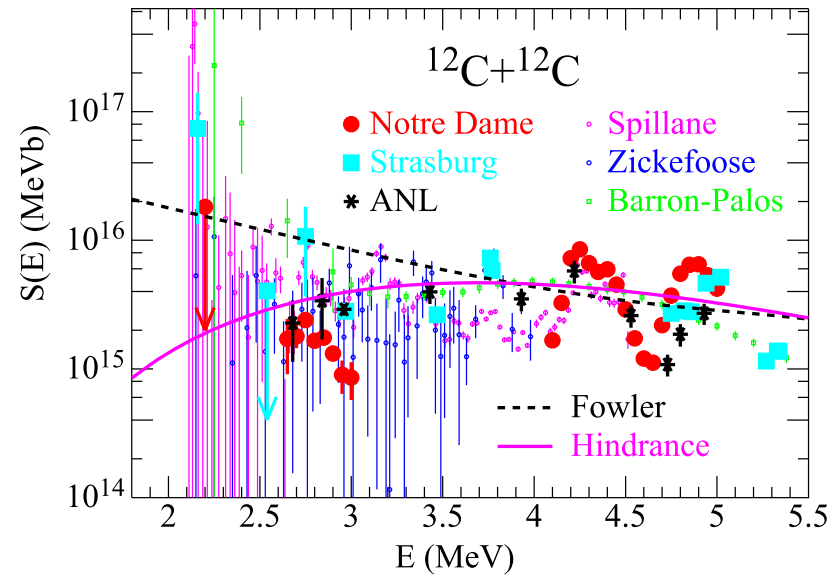

Fig. $45 S$ factor for fusion reactions in the system ${ }^{12} \mathrm{C}+{ }^{12} \mathrm{C}$ from measurements performed since 2002. The results from the most recent, particle- $\gamma$ coincidence experiments are shown by the large symbols. The results from non-coincidence experiments, detecting either chargedparticles or $\gamma$ 's (Barron-Palos, Zickefoose, and Spillane) are shown by smaller symbols

Calculating the logarithmic derivatives $L(E)$ for the ${ }^{12} \mathrm{C}+{ }^{12} \mathrm{C}$ system is quite challenging and results are shown in Fig. 46, by using cross sections from Refs. [177, 192,193]. Due to their large uncertainties, the derivatives from the Spillane and Zickefoose experiments are not shown in the figure. The cross sections from Ref. [195] were subject to a smoothing procedure and the energy range of the measurement in Ref. [196] only extends down to $4.2 \mathrm{MeV}$, so that these data are also not included. The green dashed line represents the constant $S$ factor, and the red and black dashed lines are the predictions from the hindrance model and from Fowler's parameterization, respectively. The amplitudes of the oscillations for ${ }^{12} \mathrm{C}+{ }^{12} \mathrm{C}$ are larger than those observed for ${ }^{12} \mathrm{C}+{ }^{16} \mathrm{O}$, resulting in several crossing points between the logarithmic derivative and the constant $S$-factor curve $L_{C S}$, which makes a determination of an $S$-factor maximum very uncertain.

Recently, the Trojan-Horse-Method [78] has been used as an indirect approach to constrain the fusion cross sections for ${ }^{12} \mathrm{C}+{ }^{12} \mathrm{C}$ at even lower energies [80]. The results from this experiment indicate a strong rise of the $S$ factor at energies below $2.5 \mathrm{MeV}$. However, such a large astrophysical $S$ factor is not supported by a recent new direct measurement [62] (see also Ref. [65]), as well as by the systematics deduced from the experimental data for the ${ }^{12} \mathrm{C}+{ }^{13} \mathrm{C}$ system [68]. The applicability of this approach has been questioned in several papers $[199,200]$ by pointing to the use of the PlaneWave-Approximation (PWA) instead of the Distorted-WaveBorn-Approximation. The distortion effect, which is absent in the plane-wave approximation and plays an important role when the charge of the transferred fragment $X$ of the beam, is not small $[79,199]$, where $X$ is the component of the incident 


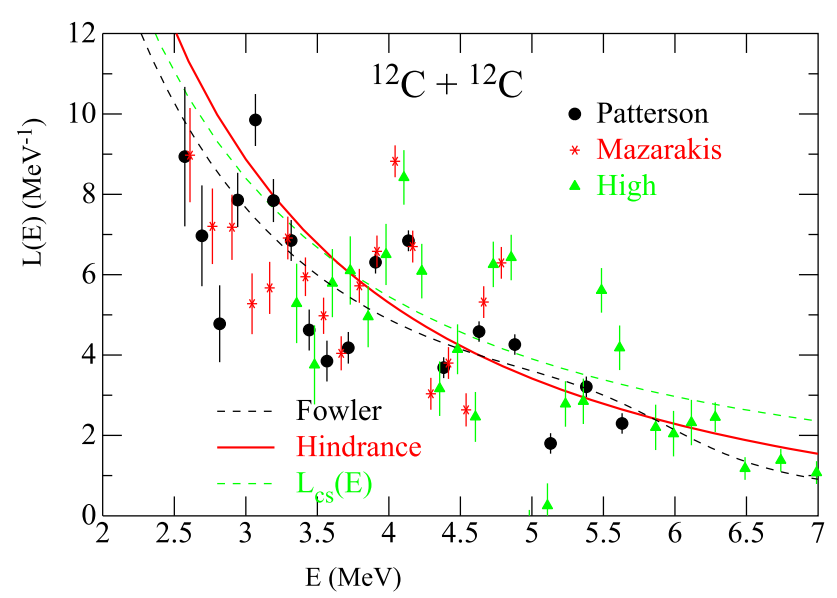

Fig. 46 Logarithmic derivative $L(E)$ for the fusion reactions in the system ${ }^{12} \mathrm{C}+{ }^{12}$ C. $L(E)$ values were obtained with the three-points difference method. The results from the most recent particle- $\gamma$ coincidence experiments are not included, since they are not measured in small enough energy steps for getting meaningful derivatives. Data from Spillane and Zickefoose are not used either, since their cross section uncertainties are very large

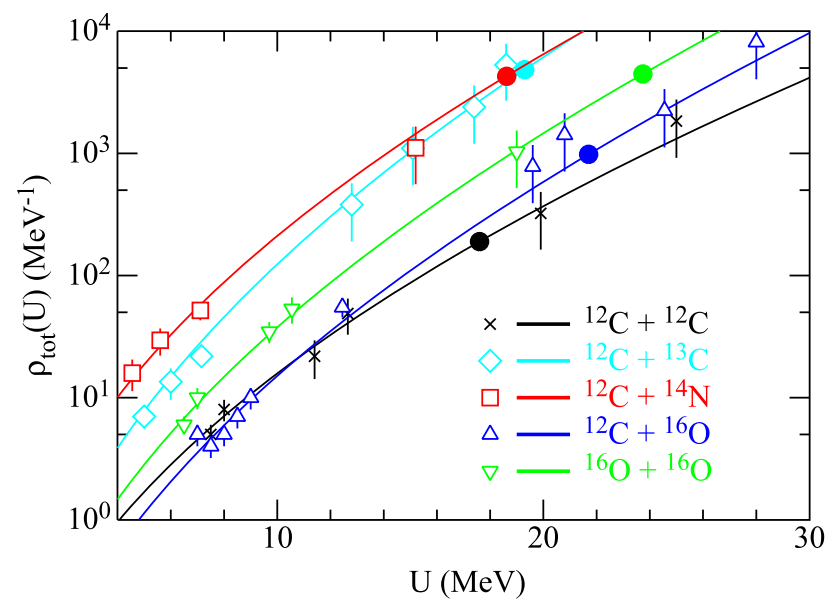

Fig. 47 Level density $\rho$ as a function of the excitation energy $U$ for the systems ${ }^{12} \mathrm{C}+{ }^{12} \mathrm{C},{ }^{12} \mathrm{C}+{ }^{13} \mathrm{C},{ }^{12} \mathrm{C}+{ }^{14} \mathrm{~N},{ }^{12} \mathrm{C}+{ }^{16} \mathrm{O}$, and ${ }^{16} \mathrm{O}+{ }^{16} \mathrm{O}$, using the parameterization of Ref. [202] and [201]. The solid points are the excitation energies at the lowest energies (measured until now) for these five systems. See text for details

nuclei, which is transfered to the target nucleus in the indirect reaction (see Fig. 10 in Sect. 2.3). This renormalization does not affect the structure of the excitation function calculated in Ref. [80], but leads to a reduction of the cross sections at $E=1.5 \mathrm{MeV}$ by more than two orders of magnitude. The astrophysical $S$ factor from the Trojan horse method in fact becomes compatible with the upper limit estimated with the sudden model [200]. A better understanding of the limitations of indirect techniques for these experiments is of paramount importance. The results of this measurement are not included in the analyses.
The results from the recent ${ }^{12} \mathrm{C}+{ }^{12} \mathrm{C}$ fusion studies can be summarized as follows: 1 ) The ${ }^{12} \mathrm{C}+{ }^{12} \mathrm{C}$ and ${ }^{12} \mathrm{C}+{ }^{16} \mathrm{O}$ systems show oscillations in their excitation functions. The other systems discussed in Section 5 as well as other fusion excitation functions e.g. ${ }^{10} \mathrm{~B}+{ }^{10} \mathrm{~B}$ (see Fig. 36), ${ }^{13} \mathrm{C}+{ }^{13} \mathrm{C}$ (Ref. [191]) and ${ }^{10} \mathrm{~B}+{ }^{12} \mathrm{C}$ [190] have smooth excitation functions that are well described by the potential model (see Fig. 41. The origin of these oscillations in the fusion of ${ }^{12} \mathrm{C}+{ }^{12} \mathrm{C}$ can be attributed to the deficit in the level densities in relation to the decay width of the compound nucleus ${ }^{24} \mathrm{Mg}$ [103]. Following the discussion from Ref. [103] by using the experimental level densities tabulated in Ref. [201] and the dependence on the excitation energy from Ref. [202] one obtains level densities for the five systems ${ }^{12} \mathrm{C}+{ }^{12} \mathrm{C},{ }^{12} \mathrm{C}+{ }^{13} \mathrm{C}$, ${ }^{12} \mathrm{C}+{ }^{14} \mathrm{~N},{ }^{12} \mathrm{C}+{ }^{16} \mathrm{O}$, and ${ }^{16} \mathrm{O}+{ }^{16} \mathrm{O}$, as shown by the solid curves in Fig. 47. The solid points give the level densities in the respective compound nuclei at the lowest measured energies. The three systems ${ }^{12} \mathrm{C}+{ }^{13} \mathrm{C},{ }^{12} \mathrm{C}+{ }^{14} \mathrm{~N}$ and ${ }^{16} \mathrm{O}+{ }^{16} \mathrm{O}$, which show smooth $S$-factor curves, have very similar level densities of $5000 / \mathrm{MeV}$ at the lowest measured energies, while the two systems with oscillations, ${ }^{12} \mathrm{C}+{ }^{16} \mathrm{O}$ and ${ }^{12} \mathrm{C}+{ }^{12} \mathrm{C}$, have lower level densities of only $200 / \mathrm{MeV}$ $\left({ }^{12} \mathrm{C}+{ }^{12} \mathrm{C}\right)$ or $1000 / \mathrm{MeV}\left({ }^{12} \mathrm{C}+{ }^{16} \mathrm{O}\right)$ at the lowest measured energy (solid points). The other parameter that needs to be included in the discussion is the width, $\Gamma_{J}$ of the respective compound level. In order to include this effect, it was suggested in Ref. [103] to impose a reduction factor $P_{J}$ on the $\mathrm{CC}(\mathrm{M} 3 \mathrm{Y}+$ rep. $)$ calculations

$\sigma=\sum_{J} \sigma_{c c}^{J} P_{J}$

where this reduction factor for the fusion cross section is given by

$P_{j}=1-\exp \left(-2 \pi \frac{\bar{\Gamma}_{J}}{D_{j}}\right)$

This reduction factor helps to explain the decrease in the average $S$ factor for energies below $E \sim 4 \mathrm{MeV}$ for ${ }^{12} \mathrm{C}+{ }^{12} \mathrm{C}$ [103].

2) By comparing the cross sections for the three systems ${ }^{12} \mathrm{C}+{ }^{12} \mathrm{C},{ }^{12} \mathrm{C}+{ }^{13} \mathrm{C}$ and ${ }^{13} \mathrm{C}+{ }^{13} \mathrm{C}$, Tang and Esbensen found that the cross sections for ${ }^{12} \mathrm{C}+{ }^{13} \mathrm{C}$ and ${ }^{13} \mathrm{C}+{ }^{13} \mathrm{C}$ provide an upper limit for the fusion cross sections of ${ }^{12} \mathrm{C}+{ }^{12} \mathrm{C}$ over a wide energy range $[120,191]$. Based on this observation a more reliable upper limit for the ${ }^{12} \mathrm{C}+{ }^{12} \mathrm{C}$ fusion cross sections at stellar energies could be established. Note, however, that it does not give the correct average behaviour of the fusion cross sections for ${ }^{12} \mathrm{C}+{ }^{12} \mathrm{C}$ at low energies. Moreover, this upper limit is much lower than what has been predicted based on the existance of a strong resonance at 1.5 $\mathrm{MeV}$, which was conjectured by Perez-Torres et al. based on an optical model calculation and used by Cooper et al. in 
an astrophysical calculation of the ${ }^{12} \mathrm{C}+{ }^{12} \mathrm{C}$ reaction rate [203,204].

3) Up to now, only the hindrance model predicts an $S$ factor maximum for ${ }^{12} \mathrm{C}+{ }^{12} \mathrm{C}$. There are many theoretical calculations, the results of which agree with the well-known Fowler curve, which exhibits an increasing $S(E)$ curve at decreasing collision energies into the stellar energy regime. However, the level density and the level width effects mentioned above have not yet been considered in many sophisticated theoretical calculations.

In a recent paper, Beck et al. have compared the two kinds of predictions, including the resonance behaviors, without reaching any conclusion on this issue [200].

Therefore, at this point it is an open question whether there is an $S$-factor maximum in ${ }^{12} \mathrm{C}+{ }^{12} \mathrm{C}$ fusion, although the three latest results of particle- $\gamma$ coincidence experiments are in agreement with the hindrance model. It may be noted that the results at low energies around $2-3 \mathrm{MeV}$ obtained by Zickefoose [58] in the most recent singles measurement do not support the theoretical curves mentioned above, which predict a strong increase of $S$-factor towards low energies.

\subsection{Fusion hindrance for astrophysical systems.}

The systematics of fusion hindrance covering the full range from light to heavy systems will be discussed in the next subsection. Due to the importance of fusion reactions for nuclear astrophysics, we will here give some general comments about the existence or non-existence of fusion hindrance. The experimental data presented for many heavier systems in Sect. 4 show the existence of fusion hindrance through the presence of an $S$-factor maximum.

These maxima follow a systematic trend which can be used to make extrapolations for lighter systems where no maximum has yet been observed. Since, as shown in Fig. 48, there is some nuclear structure dependence of the $E_{S}$ values with closed-shell nuclei having the maximum at higher energies when compared to open-shell nuclei, the extrapolated values for light nuclei can fluctuate by $10-15 \%$. For the astrophysically important systems, the predicted values range from 6.3 to $7.3 \mathrm{MeV}$ for ${ }^{16} \mathrm{O}+{ }^{16} \mathrm{O}, 4.2-4.9 \mathrm{MeV}$ for ${ }^{16} \mathrm{O}+{ }^{12} \mathrm{C}$ and $3.4-4.0 \mathrm{MeV}$ for ${ }^{12} \mathrm{C}+{ }^{12} \mathrm{C}$. While the above value for ${ }^{16} \mathrm{O}+{ }^{16} \mathrm{O}$ does cover the measured $E_{s}$ observed in experiments, the values of the other two systems are located at the low experimental regions and the oscillations complicate the determination and the debate about the $S$-factor maximum.

It should be mentioned that the extrapolation formula, $L(E)=A_{0}+B_{0} / E^{3 / 2}$, is an empirical one. If the exponent $3 / 2$ in the expression changes to a smaller value, the extrapolation of $S(E)$ will not give a maximum. While this expression works well for systems as light as ${ }^{12} \mathrm{C}+{ }^{16} \mathrm{O}$ (see Sect. 5.3), it might not be appropriate for all light systems.
Table 1 Summary of energies $E_{S}$ at the $S$-factor maxima. The system, fusion $Q$ value, the parameter $\zeta=Z_{1} Z_{2} \sqrt{M_{1} M_{2} /\left(M_{1}+M_{2}\right)}$, that characterizes the system and the energy $E_{S}$ are included in column 14 , respectively. The first group shows the medium-mass and heavy stiff (closed-shell) systems, while the second groups shows the soft systems. The third and fourth groups include medium-mass and light systems, respectively. For the first three groups, an $S$-factor maximum has been observed in the experiments. For the systems in the fourth group no $S$-factor maximum has been observed yet, and the $E_{S}$ value has been obtained by extrapolation

\begin{tabular}{|c|c|c|c|c|}
\hline System & $Q$ value $\mathrm{MeV}$ & $\zeta$ & $E_{s} \mathrm{MeV}$ & References \\
\hline${ }^{90} \mathrm{Zr}+{ }^{92} \mathrm{Zr}$ & -153.7 & 10786 & 170.7 & {$[33]$} \\
\hline${ }^{90} \mathrm{Zr}+{ }^{90} \mathrm{Zr}$ & -157.3 & 10727 & 175.2 & {$[33]$} \\
\hline${ }^{90} \mathrm{Zr}+{ }^{89} \mathrm{Y}$ & -151.5 & 10430 & 170.8 & {$[33]$} \\
\hline${ }^{60} \mathrm{Ni}+{ }^{89} \mathrm{Y}$ & -90.49 & 6533 & 122.9 & [11] \\
\hline${ }^{58} \mathrm{Ni}+{ }^{58} \mathrm{Ni}$ & -65.85 & 4219 & 94.0 & [32] \\
\hline${ }^{54} \mathrm{Fe}+{ }^{58} \mathrm{Ni}$ & -56.51 & 3848 & 86.7 & {$[46]$} \\
\hline${ }^{19} \mathrm{~F}+{ }^{208} \mathrm{~Pb}$ & -50.07 & 3079 & 75.5 & [205] \\
\hline${ }^{32} \mathrm{~S}+{ }^{89} \mathrm{Y}$ & -36.62 & 3026 & 73.1 & [164] \\
\hline${ }^{16} \mathrm{O}+{ }^{208} \mathrm{~Pb}$ & -46.48 & 2528 & 68.0 & {$[110]$} \\
\hline${ }^{12} \mathrm{C}+{ }^{198} \mathrm{Pt}$ & -13.96 & 1574 & 48.0 & [70] \\
\hline${ }^{11} \mathrm{~B}+{ }^{197} \mathrm{Au}$ & -5.00 & 1275 & 39.8 & [206] \\
\hline${ }^{64} \mathrm{Ni}+{ }^{100} \mathrm{Mo}$ & -92.26 & 7343 & 120.6 & {$[143]$} \\
\hline${ }^{64} \mathrm{Ni}+{ }^{64} \mathrm{Ni}$ & -48.80 & 4432 & 87.5 & {$[12]$} \\
\hline${ }^{36} \mathrm{~S}+{ }^{64} \mathrm{Ni}$ & -8.54 & 2149 & 52.3 & [165] \\
\hline${ }^{28} \mathrm{Si}+{ }^{64} \mathrm{Ni}$ & -1.79 & 1729 & 45.6 & {$[162]$} \\
\hline${ }^{27} \mathrm{Al}+{ }^{45} \mathrm{Sc}$ & 9.63 & 1121 & 32.4 & {$[172]$} \\
\hline${ }^{28} \mathrm{Si}+{ }^{30} \mathrm{Si}$ & 14.30 & 745.6 & 24.7 & {$[161]$} \\
\hline${ }^{24} \mathrm{Mg}+{ }^{30} \mathrm{Si}$ & 17.89 & 613.2 & 20.8 & [207] \\
\hline${ }^{12} \mathrm{C}+{ }^{30} \mathrm{Si}$ & 14.11 & 245.9 & 10.5 & {$[141]$} \\
\hline${ }^{12} \mathrm{C}+{ }^{24} \mathrm{Mg}$ & 16.30 & 203.6 & 9.7 & [174] \\
\hline${ }^{16} \mathrm{O}+{ }^{16} \mathrm{O}$ & 16.54 & 181.0 & 6.78 & $\overline{[180-184]}$ \\
\hline${ }^{12} \mathrm{C}+{ }^{20} \mathrm{Ne}$ & 18.97 & 164.3 & 5.85 & [180] \\
\hline${ }^{14} \mathrm{~N}+{ }^{16} \mathrm{O}$ & 18.33 & 153.0 & 5.39 & [208] \\
\hline${ }^{14} \mathrm{~N}+{ }^{14} \mathrm{~N}$ & 27.23 & 129.6 & 4.15 & [209] \\
\hline${ }^{13} \mathrm{C}+{ }^{16} \mathrm{O}$ & 20.28 & 128.6 & 4.00 & [189] \\
\hline${ }^{12} \mathrm{C}+{ }^{16} \mathrm{O}$ & 16.76 & 125.7 & 4.54 & {$[187,188]$} \\
\hline${ }^{12} \mathrm{C}+{ }^{14} \mathrm{~N}$ & 15.07 & 106.8 & 5.09 & [190] \\
\hline${ }^{12} \mathrm{C}+{ }^{13} \mathrm{C}$ & 16.32 & 89.9 & 3.45 & {$[90,191]$} \\
\hline${ }^{12} \mathrm{C}+{ }^{12} \mathrm{C}$ & 13.93 & 88.2 & 3.68 & [192-194] \\
\hline${ }^{11} \mathrm{~B}+{ }^{14} \mathrm{~N}$ & 24.72 & 86.9 & 2.90 & [210] \\
\hline${ }^{11} \mathrm{~B}+{ }^{12} \mathrm{C}$ & 18.20 & 71.9 & 2.12 & {$[190]$} \\
\hline${ }^{10} \mathrm{~B}+{ }^{10} \mathrm{~B}$ & 31.14 & 55.9 & 1.47 & [177] \\
\hline
\end{tabular}

\section{Systematics of hindrance effect}

\subsection{Systematics of $E_{s}$ at the $S$-factor maximum}

In order to obtain a reference curve for the energy, $E_{s}$, at the $S$-factor maximum, the measured $E_{S}$ values for stiff (closed- 
shell), medium-heavy and heavy systems are summarized in the first part of Table 1. The second part lists the measured $E_{S}$ values for some soft systems in the same mass region, whereas the third part gives measured values for mediummass systems. The fourth part summarizes light-mass systems, where the $E_{S}$ values are obtained by extrapolation using Eqs. (25) and (28) under the assumption that there are also $S$-factor maxima.

We note that the first three systems listed in Table 1 are heavy and they may therefore have a non-vanishing fission decay branch. Since this decay branch has not been measured for these systems, caution should be exercized in interpreting the results for $E_{s}$ given in Table 1 based solely on the measured ER cross sections. However, the fact that the $E_{S}$ values for these systems fall well in line with the overall systematics (see Fig. 48) indicates that the fission branch is not a major contributor. In the subbarrier region statistical model calculations using the PACE4 code support this conclusion.

In the lower panel of Fig. 48 we show the $E_{S}$ values plotted as a function of the system parameter $\zeta$ [(defined in Eq. (21)] for all the systems listed in Table 1 . The symbols refer to the four groups of the Table, i.e. blue stars for light, red circles for medium-light, green circles for medium-mass soft, and black for medium-heavy and heavy stiff systems. In the upper panel, the corresponding, experimental logarithmic slopes $L_{S}\left(E_{S}\right)=0.495 \zeta / E_{s}^{3 / 2}\left(\mathrm{MeV}^{-1}\right)$ and $E_{S}$ is given in units of $(\mathrm{MeV})$ are shown with the same symbols.

The magenta curves in Fig. 48 are obtained from leastsquares fit of $L_{s}$ vs. $\zeta$ for the systems listed in the first, third and fourth parts with the result

$L_{S}(\zeta)=2.19+511 / \zeta\left(\mathrm{MeV}^{-1}\right)$

which deviates slightly from the historical value of 2.33 $\mathrm{MeV}^{-1}$, Ref. [13]. The corresponding magenta curve for $E_{s}$ is given by

$$
E_{S}(\zeta)=[0.495 \zeta /(2.19+511 / \zeta)]^{2 / 3}(\mathrm{MeV})
$$

which provides an average description of the stiff systems over the whole mass region while the soft systems listed in second part of the Table, such as e.g. ${ }^{64} \mathrm{Ni}+{ }^{64} \mathrm{Ni}$ and ${ }^{64} \mathrm{Ni}+{ }^{100} \mathrm{Mo}$, lie below the curve. Correspondingly, the curve for $L_{S}(\zeta)$ gives an average description of the stiff systems, while the soft systems lie above the curve.

It is clear that the ${ }^{12} \mathrm{C}+{ }^{24} \mathrm{Mg},{ }^{30} \mathrm{Si}$ systems deviate from these systematics. This will be discussed later.

\subsection{Radius of curvature $\rho$ of $S(E)$ near the $S$-factor maximum, $A_{0}$ and $B_{0}$}

In Ref. [211], the slopes of the logarithmic derivative were studied quantitatively. The ratio of the slopes of $L(E)$ and

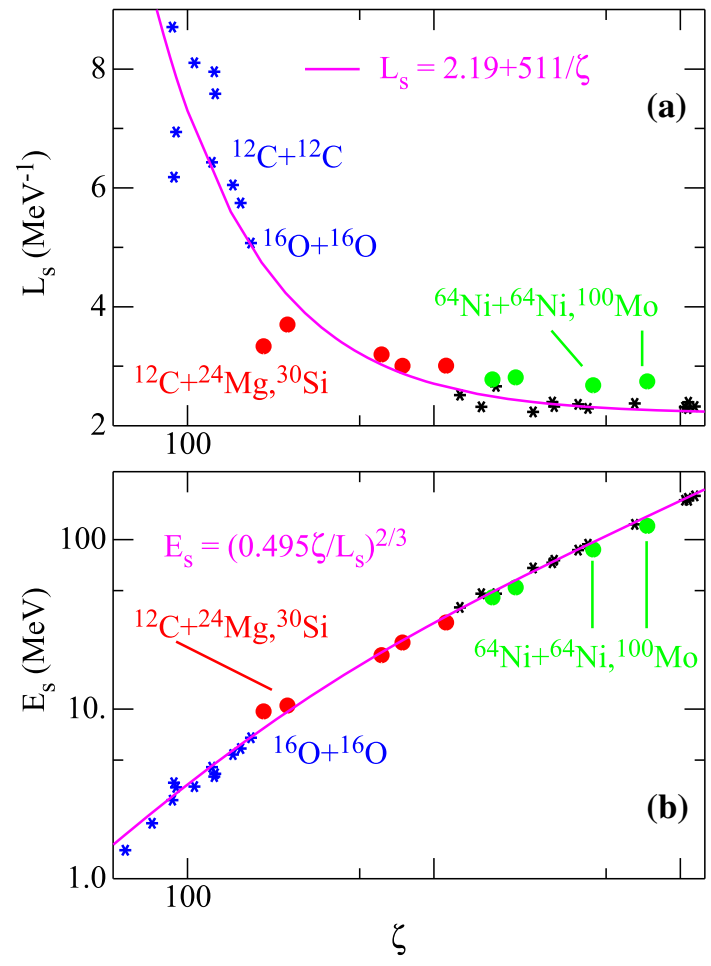

Fig. 48 a The logarithmic derivative of the excitation function at the hindrance threshold and $\mathbf{b}$ the energy at the maximum of the $S$ factor are plotted vs. the system parameter $\zeta$, for the systems of Table 1 . The magenta lines are the result of least-squares fits, as specified in the two panels, obtained by considering only the first-, third- and fourth-group systems in Table 1 . The fitted curves have parameters very close to those obtained in the previous papers e.g. Ref. [175]

$L_{c s}(E)$,

$R R=\frac{d L(E) / d E}{d L_{c s}(E) / d E}$.

is a quantity related to the curvature of the $S$-factor maximum. A value of $R R=1$, means that the two curves, $L(E)$ and $L_{c s}(E)$, are parallel. In Fig. 49, the experimental results for the ratio $R R$ are shown. The black symbols originate from Ref. [211], whereas the red points are from recent measurements for the systems ${ }^{12} \mathrm{C}+{ }^{24} \mathrm{Mg}$ [174], ${ }^{12} \mathrm{C}+{ }^{30} \mathrm{Si}$ [141], ${ }^{24} \mathrm{Mg}+{ }^{30} \mathrm{Si}[173],{ }^{28} \mathrm{Si}+{ }^{30} \mathrm{Si}[161],{ }^{30} \mathrm{Si}+{ }^{30} \mathrm{Si}[163,219]$, ${ }^{27} \mathrm{Al}+{ }^{45} \mathrm{Sc}$ [172] and ${ }^{40} \mathrm{Ca}+{ }^{48} \mathrm{Ca}$ [163]. The open symbols are obtained by using extrapolation to low energies with the empirical expressions Eqs. (25-28) in Sect. 4.3. The black curve is a second-order polynomial of $\zeta$ given in Ref. [211],

$R R=1.10+1.70 \times 10^{-3} \zeta+9.48 \times 10^{-8} \zeta^{2}$.

As can be seen from Fig. 49, $R R$ decreases and approaches to a value near unity as $\zeta$ decreases.

Approximating the $S$ factor near the maximum by a Gaussian curve,

$S(E)=S_{\text {max }} \exp \left[-\left(E-E_{s}\right)^{2} / 2 \rho^{2}\right]$, 


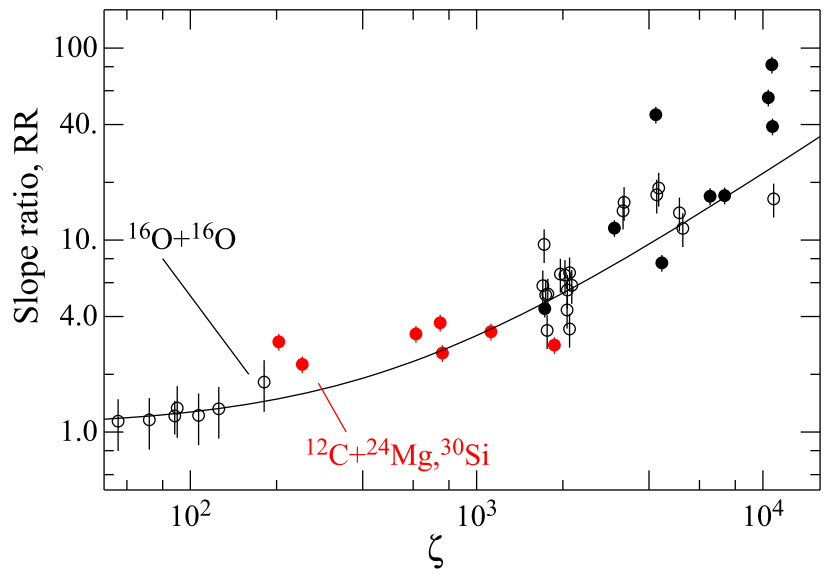

Fig. $49 R R$, the ratio of energy derivatives of the slopes $L(E)$ and $L_{c s}(E)$ at their crossing points is shown as a function of the system parameter $\zeta$. The solid circles are extracted from experimental data while the open ones are extrapolated from data at low energies. The red circles are new measurements performed since the publication of Ref. [211]. The curve is a polynomial fit to the black data points

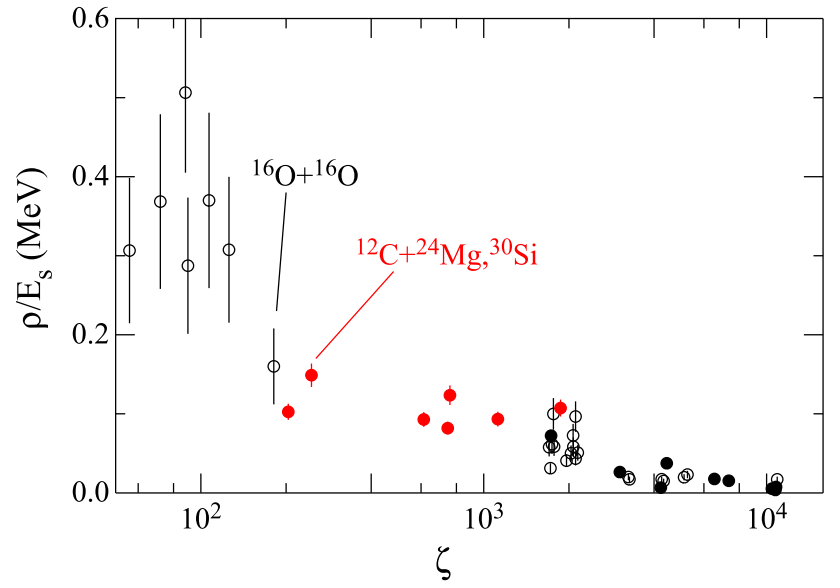

Fig. 50 Plot of the ratio $\rho / E_{s}$ as a function of the system parameter $\zeta$. The solid circles are extracted from experimental data while the open symbols are extrapolated from data at low energies. The red circles are the results from new measurements published after Ref. [211]

the variance $\rho$ of the distribution is given by

$\frac{1}{\rho}=\left|\frac{d^{2} S}{d E^{2}}\right|=\left|\frac{d L}{d E}-\frac{d(\pi \eta / E)}{d E}\right|$, at $E=E_{s}$.

It can be used as a measure of the sharpness of the $S$-factor maximum. $1 / \rho$ is determined by the difference of the derivatives of $L(E)$ and $L_{c s}(E)$.

In Fig. 50 we show the results for the ratio $\rho / E_{s}$ as a function of $\zeta$. A pronounced $S$-factor maximum appears for small values of $\rho / E_{S}$, whereas the $S(E)$ factor is wider near the $S$-factor maximum for larger values of $\rho / E_{S}$,

In addition to the difficulties in identifying an $S$-factor maximum because of a gentle cross-over between the $L(E)$ and $L_{c s}(E)$ curves, these difficulties are aggravated by poor

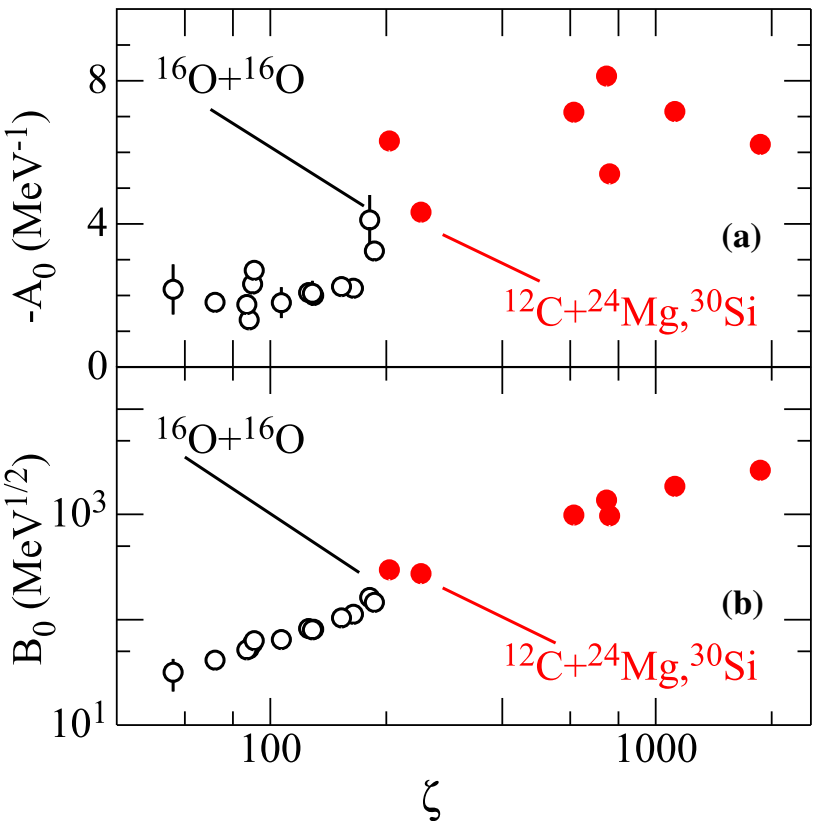

Fig. 51 Systematics of the fit parameters $A_{0}$ and $B_{0}$ as a function of $\zeta$

statistics and the serious backgrounds associated with the small cross sections in the sub-barrier region. It is an experimental challenge to ascertain whether the $S$-factor maximum exists in light systems, and the problem becomes more and more serious as the mass of compound nucleus decreases.

In Fig. 51, the parameters $A_{0}$ and $B_{0}$ which describe the dependence of the logarithmic derivative, $L(E)$, as a function of collision energy (see Eq. 25) are plotted as a function of $\zeta$. While for the parameter $B_{0}$ as a function of $\zeta$ a nice systematic trend is observed, the parameter $A_{0}$ shows larger fluctuations, especially for heavier systems. Low-mass systems, which are of interest in nuclear astrophysics, have an almost constant value of $A_{0}=-2.2$.

\subsection{Strength of fusion enhancement and hindrance}

The signatures of fusion hindrance in lighter systems (e.g. ${ }^{12} \mathrm{C}+{ }^{24} \mathrm{Mg}$ or ${ }^{12} \mathrm{C}+{ }^{30} \mathrm{Si}$ ) are less pronounced than the ones in heavier systems (e.g. those presented in Sect. 4). This behavior is shown in more detail in Fig. 52 by plotting the ratios between the experimental cross sections and the the standard CC calculations with a Woods Saxon potential including all couplings, which is denoted $\sigma_{\mathrm{cc}-\text { ws }}$ as a function of $E-V_{c}$. Here $V_{c}$ is the Coulomb barrier calculated with the Akyüz-Winther potential [150]. The resulting cross section ratios are shown in the upper panel for several systems from ${ }^{64} \mathrm{Ni}+{ }^{64} \mathrm{Ni}$ to ${ }^{12} \mathrm{C}+{ }^{30} \mathrm{Si}$. One can clearly see that fusion hindrance is present by the fact that this ratio shows a pronounced deviation below unity in medium-mass systems, whereas the deviation is smaller for ${ }^{12} \mathrm{C}+{ }^{30} \mathrm{Si}$. It should 

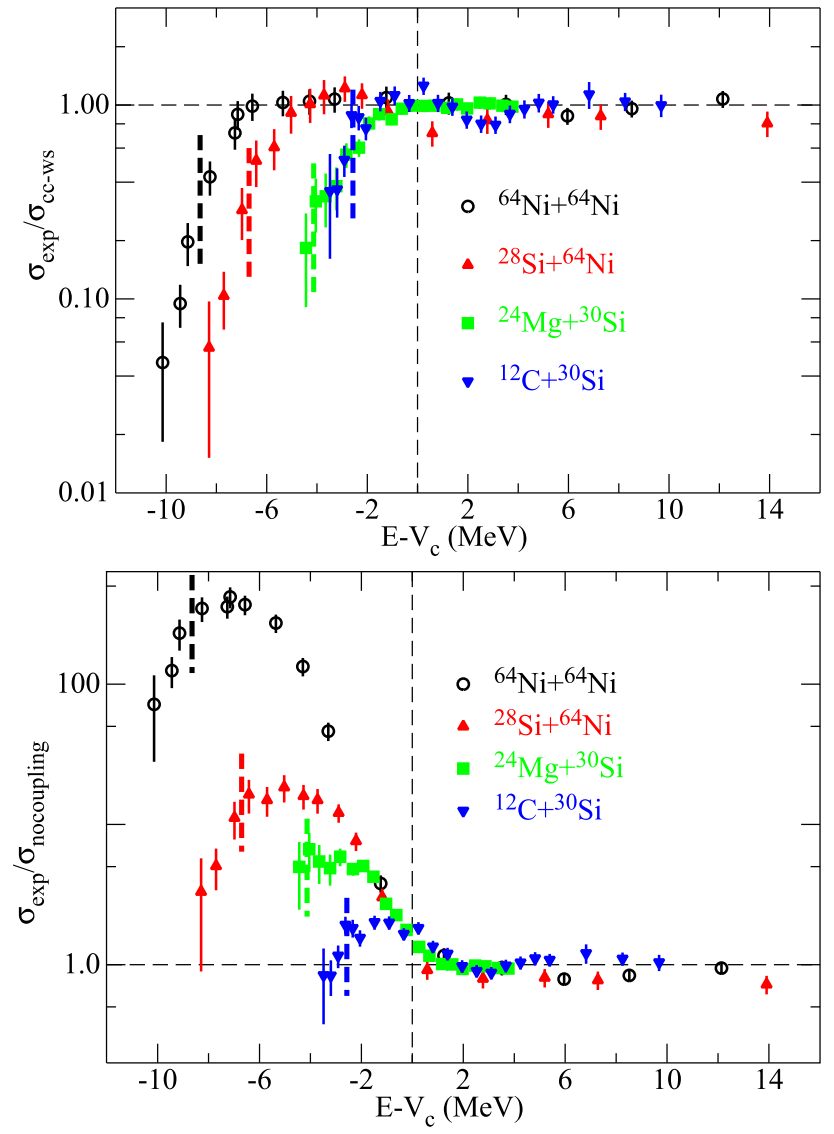

Fig. 52 Upper panel: ratio of the experimental cross sections to the $\mathrm{CC}$ calculations using the Woods-Saxon potential for different systems. Lower panel: comparison done with the no-coupling limit for the same systems. Values of $V_{c}$ are $96.15,52.30,24.73$ and $13.07 \mathrm{MeV}$ for ${ }^{64} \mathrm{Ni}+{ }^{64} \mathrm{Ni},{ }^{28} \mathrm{Si}+{ }^{64} \mathrm{Ni},{ }^{24} \mathrm{Mg}+{ }^{30} \mathrm{Si}$, and ${ }^{12} \mathrm{C}+{ }^{30} \mathrm{Si}$, respectively

be noted, however, that the two heavier systems have been measured down to smaller cross sections with respect to the two lighter systems.

For comparison, the ratios between the experimental cross sections and a calculation using a Woods Saxon potential but without couplings, $\sigma_{\text {nocoupling }}$ are shown in the lower panel. These ratios display a maximum below the Coulomb barrier but typically $2 \mathrm{MeV}$ above the hindrance threshold indicated by the vertical dashed lines. The peak is much more prominent for heavier systems, indicating that the cross section enhancement caused by coupled-channels effects is very strong in those heavier cases.

Obviously, stronger enhancement and stronger hindrance show up together for medium-mass systems. One can understand that the two phenomena are not independent since they have a common underlying physics cause as suggested by Ichikawa et al. $[126,127]$ with strenghs that scale with the factor $Z_{1} Z_{2}$.

At lower energies the ratio $\sigma_{\exp } / \sigma_{\text {nocoupling }}$ decreases in all cases, and reaches unity for ${ }^{12} \mathrm{C}+{ }^{30} \mathrm{Si}$. New experimen-
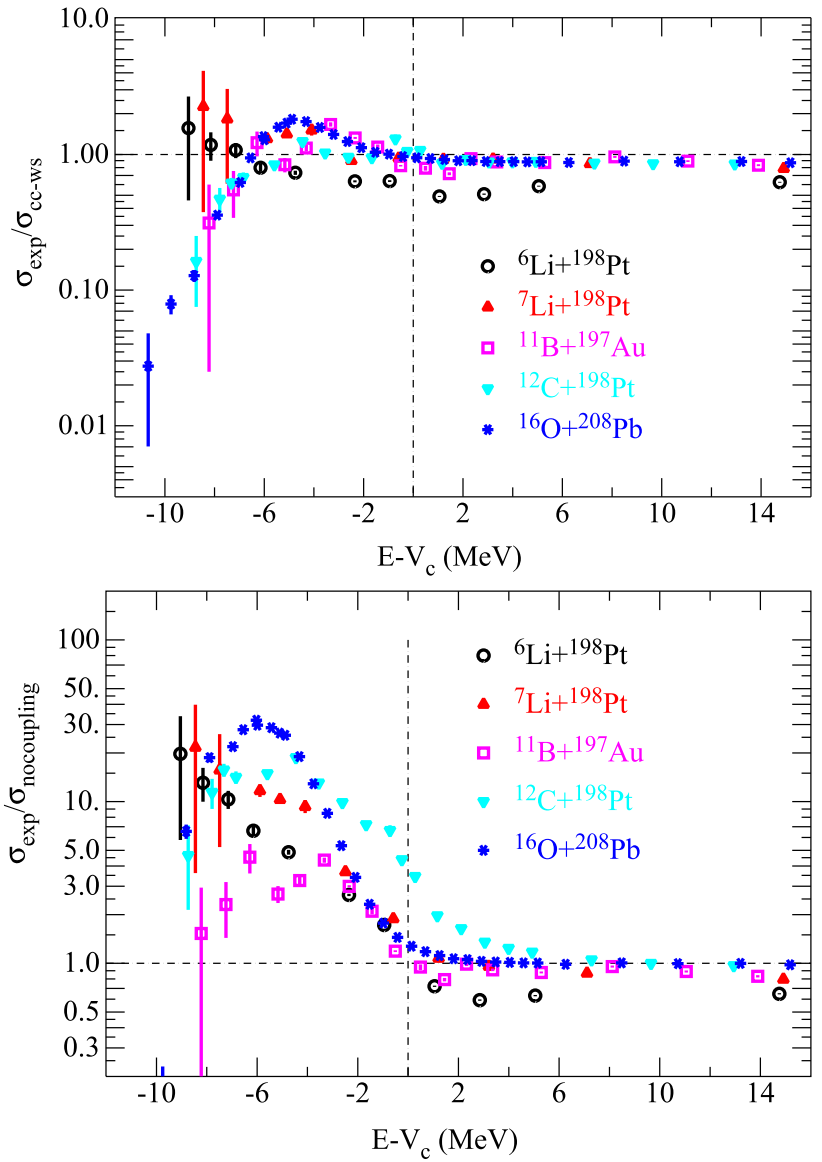

Fig. 53 Upper panel: measured vs. calculated fusion cross sections as a function of the energy relative to the Coulomb barrier for ${ }^{6,7} \mathrm{Li}+{ }^{198} \mathrm{Pt}$ $[69,70],{ }^{12} \mathrm{C}+{ }^{198} \mathrm{Pt}[70],{ }^{16} \mathrm{O}+{ }^{208} \mathrm{~Pb}[110,142]$. In the calculations a standard WS potential was used. Lower panel: the same experimental data are compared to the analogous no-coupling calculations. $V_{c}$ values are 28.55, 28.49, 47.34, 55.88 and $76.52 \mathrm{MeV}$ for ${ }^{6} \mathrm{Li}+{ }^{198} \mathrm{Pt},{ }^{7} \mathrm{Li}+{ }^{198} \mathrm{Pt}$, ${ }^{11} \mathrm{~B}+{ }^{197} \mathrm{Au},{ }^{12} \mathrm{C}+{ }^{198} \mathrm{Pt}$, and ${ }^{16} \mathrm{O}+{ }^{208} \mathrm{~Pb}$, respectively

tal data could clarify whether the cross section approaches the no-coupling limit, i.e. $\sigma_{\exp } / \sigma_{\text {nocoupling }} \sim 1$ at extreme sub-barrier energies, or whether the decrease of the ratio will continue at lower energies, possibly revealing new information about the ion-ion potential far below the barrier.

Several asymmetric systems were recently studied by Shrivastava et al. [70]. They investigated the features of fusion hindrance and how it evolves with increasing mass and charge for light projectiles such as ${ }^{6,7} \mathrm{Li}$ and ${ }^{12} \mathrm{C}$ on the heavy target ${ }^{198} \mathrm{Pt}$. In their studies, the case of ${ }^{16} \mathrm{O}+{ }^{208} \mathrm{~Pb}$ $[110,142]$ was also re-analysed. Fusion cross sections down to very small values $(\approx 100 \mathrm{nb})$ were measured using an offline technique by detecting coincidences between characteristic $\mathrm{X}$ - and $\gamma$-rays (see also [71]).

Similar to Fig. 52, ratios for these asymmetric systems are shown in Fig. 53. From the upper panel of Fig. 53, clearly, fusion hindrance is not observed for ${ }^{6,7} \mathrm{Li}$, but shows up when going to the heavier ${ }^{12} \mathrm{C}$ and ${ }^{16} \mathrm{O}$ projectiles. The different 
behaviour of the ${ }^{6,7} \mathrm{Li}$-induced reactions might be due to competing reaction channels such as transfer and/or break up, preventing the observation of fusion hindrance. A comparison of the lower panels of Figs. 52 and 53 also indicates that the cross section enhancement observed for the very asymmetric systems induced by light projectiles is much smaller than the value observed for medium-mass systems such as e.g. ${ }^{64} \mathrm{Ni}+{ }^{64} \mathrm{Ni}$. These observed trends indicate the importance of investigating the behaviour of light and more asymmetric systems where the existence of hindrance and its features are uncertain and a matter of debate based on the few data, which are presently available.

\section{Simple analytic fusion cross section formulae}

In this section we give an overview of various analytic equations that have been suggested for the description of fusion excitation functions.

\subsection{Logarithmic derivative fits}

In order to describe the fusion hindrance at extreme subbarrier energies and to extrapolate the excitation function to lower energies, empirical expressions of the excitation functions, Eqs. (25-28), that fit the logarithmic derivative data at low energies have been introduced (see [170,171]). The $S$-factor maximum is also well reproduced by this empirical parametrization.

\subsection{The Wong formula}

It is well known that the Wong formula [27] given in Eq. 3 can reproduce excitation functions at higher energies quite accurately, but that it often overpredicts the cross sections in the range below $\sim 0.1 \mathrm{mb}$.

The derivation of the Wong formula is based on two assumptions, namely (1) that the fusion barrier shape is approximated by an inverted parabola for which the HillWheeler formula for quantum mechanical tunneling can be applied, and (2) that the radius, $R$ and curvature $\hbar \omega$ of the fusion barrier is independent of the angular momentum. It has been found that, because of the first assumption, this formula fails to reproduce the fusion hindrance seen at deep subbarrier energies because the inverted parabola is not a good approximation to the actual shape of the fusion barrier far below its maximum. Thus, the $1 / r$ dependence of the Coulomb repulsion at larger distances combined with the approximate exponential shape of the nuclear attraction at smaller distances yield a less penetrable barrier than the inverted parabola assumed in the Wong formula. It has recently been shown also that the second assumption is problematic, especially for light systems. Recently, various modifications to the Wong formula have been proposed to alleviate these shortcomings as discussed in the next subsection.

At energies higher than the Coulomb barrier, the Wong formula approaches the expression, Eq. 2 for the black body approximation [26].

\subsection{Modifications of the Wong formula}

As mentioned above, in deriving his formula, Wong relied on the assumptions that $R$ and $\hbar \omega$ are independent of the angular momentum $l$. Recently, however, Rowley and Hagino presented an analysis [212] which shows that the parameters $B, R, \omega$ should be angular momentum dependent. They suggested to use a different parameter set for each angular momentum $l$, and that one needs to derive numerically the parameters, $V_{E}, R_{E}$ and $\omega_{E}$.

In an alternative approach, Denisov and Sedykh recently proposed to include a collision-energy dependence of the barrier curvature and developed a method to obtain empirical relations for the parameters $V, R$ and $\hbar \omega$ in the Wong formula [18], where $V$ is the barrier height. In this empirical description, Denisov et al. assumed that $\hbar \omega$ includes an energy dependent factor $f(E)$ given by

$f(E)=\frac{(E / V)^{3}}{1+\exp [-0.14215(E-V)]}$.

A global fit to 85 experimental excitation functions of even-even systems was carried out in order to fix nine free parameters in their formulation. These fit results (85 figures) have been presented in Ref. [18]. In Figs. 54 and 55 we show the results for ${ }^{16} \mathrm{O}+{ }^{208} \mathrm{~Pb}$ and ${ }^{64} \mathrm{Ni}+{ }^{64} \mathrm{Ni}$, respectively, compared with the calculations by the Denisov model shown by the red curves. Both calculations are found to be in fair agreement with the data and predict an $\mathrm{S}$-factor maximum, albeit not at the correct energies.

One should keep in mind, however, that in this approach only one set of nine global parameters is used. Other calculations, using parameters individually adjusted to a particular system are shown by the black $(L(E)$ fit) and green curves (single-Gaussian fit), will be discussed in next subsection.

\subsection{Gaussian barrier distribution}

The coupling between the fusion channel and intrinsic excitations of the interacting nuclei, as described in the coupled channels approach, leads to a distribution of interaction barriers, which for most cases results in an enhanced cross section at energies below the uncoupled fusion barrier. Rowley et al. [10] have shown that this distribution of barrier heights, $D(E)$, can be extracted from experimental fusion data under certain assumptions, provided that the cross sections are sufficiently precise and that the excitation function has been measured in small energy steps, such that the second deriva- 


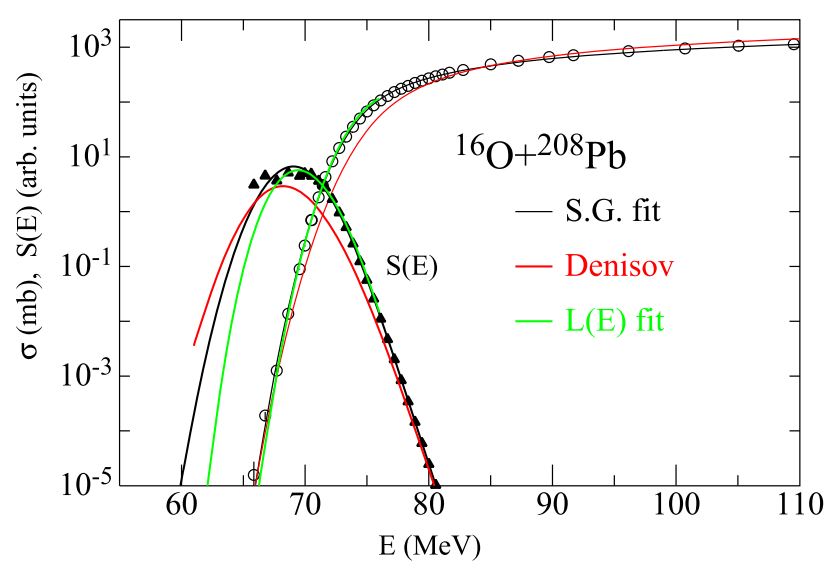

Fig. 54 Fusion cross sections, $\sigma(E)$ (open circles) and the corresponding $S(E)$ factors (solid triangles) for the system ${ }^{16} \mathrm{O}+{ }^{208} \mathrm{~Pb}[110]$. The red curve is the result from the Denisov model, whereas the black and green curves are fits to the data using the single-Gaussian (Eq. 41) and the logarithmic derivative (Eqs. 25, 26) approach, respectively

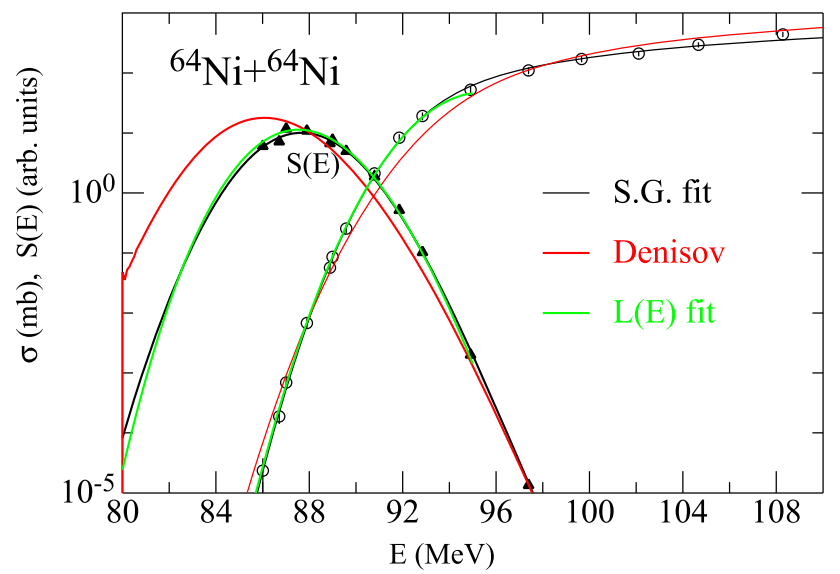

Fig. 55 Fusion cross sections, $\sigma(E)$ (open circles) and the corresponding $S(E)$ factors (solid triangles) are shown for the system ${ }^{64} \mathrm{Ni}+{ }^{64} \mathrm{Ni}$ [12]. The red curve is the result from Denisov model, whereas the black and green curves are fits to the data using the single-Gaussian (Eq. 41) and the logarithmic derivative (Eqs. 25, 26), respectively

tive of the product of the cross section and the collision energy can be computed with sufficient accuracy. In this case, the distribution of fusion barriers can be derived from

$D(E)=\frac{1}{\pi R^{2}} \frac{d^{2}(\sigma(E) E)}{d E^{2}}$, with $\int D(E) d E=1$

Subsequently, barrier distributions for many systems have been studied using this method $[2,10]$. This kind of analysis has played an important role for understanding the dynamics of heavy-ion fusion reactions. One must emphasize, however, that although Eq. (40) does not include any approxitation, the interpretation of $D(E)$ as a barrier distribution was obtained under the black body approximation.
Another cross section formula was proposed by SiwekWilczynska et al. [213,214], given by

$\sigma_{g}(E)=\frac{\sqrt{\pi} R_{g}^{2} W_{g}}{\sqrt{2} E}\left[\sqrt{\pi} Z \operatorname{erfc}(-Z)+\exp \left(-Z^{2}\right)\right]$,

where $Z=\left(E-V_{g}\right) / \sqrt{2} W_{g}$ and $R_{g}$ is the barrier radius. The quantities $V_{g}$ and $W_{g}$ are defined below. This expression was originally used in their study of super-heavy elements formation, and was subsequently applied to the analysis of deep subbarrier fusion reactions by Jiang et al. [17]. This approach includes the effects of Coupled-channels in a schematic way by approximating the resulting barrier distribution by a Gaussian function and gives a surprisingly good representation of the subbarrier hindrance phenomenon. In the following, we discuss the essential points in more detail.

Siwek-Wilczynska et al. started from a Gaussian fusion barrier distribution $D_{g}(V)$ and followed the idea by Stelson et al. [215] and Rowley et al. [10] of using the black body approximation [26] to arrive at the expressions

$D_{g}(V)=\frac{1}{\sqrt{2 \pi} W_{g}} \exp \left[-\left(\frac{V-V_{g}}{\sqrt{2} W_{g}}\right)^{2}\right]$,

with

$\sigma(E)=\frac{\pi R^{2}}{E} \int_{E_{0}}^{E}(E-V) D_{g}(V) d V$.

Here, $V$ is the barrier height, $V_{g}$ is the centroid and $W_{g}$ the standard deviation of the distribution. The lower integration limits are $E_{0}=-Q$ or 0 , depending on whether the fusion $Q$ value is negative or positive. Finally, they obtained a simple, analytic function for the cross section, Eq. 41, which includes the complementary error function $\operatorname{erfc}(Z)$.

It should be emphasized, however, that the derivation of this formula relies on the assumption that for each barrier height in the assumed distribution fusion occurs only if the collision energy exceeds the barrier height for the partial wave in question, i.e. there is no quantum mechanical tunneling of the barrier. The sub-barrier behavior is thus entirely dependent on the shape of the barrier distribution at these energies.

In Ref. [214], a table was included for 46 systems heavier than ${ }^{48} \mathrm{Ca}+{ }^{48} \mathrm{Ca}$, for which relevant barrier distribution parameters were derived. The data used were mostly in the cross section range from 0.1 to $1000 \mathrm{mb}$.

In Ref. [17] it was found that Eq. (41) can reproduce the hindrance behavior at extreme subbarrier energies very well, even better than predicted by many theoretical calculations.

\subsection{Comparison with experimental data}

Although there are more than one thousand of experimental fusion excitation functions published in the literature, only 
Table 2 Parameters obtained by least-squares fits with single-Gaussian and $L(E)$ recipes for 35 fusion systems, whose lowest measured cross sections are less than $0.02 \mathrm{mb}$. $Q$ is the fusion $Q$ value, $\mathrm{N}$ is the number of data points in the fitting, $\chi^{2}$ is the value defined in Eq. 44, EVR and FF are cross sections for fusion-evaporation residues and fusion-fission, respectively. See text for details

\begin{tabular}{|c|c|c|c|c|c|c|c|c|c|c|c|c|c|c|}
\hline System & $\begin{array}{l}Q \\
\mathrm{MeV}\end{array}$ & Type & $N$ & $\begin{array}{l}\text { Data range } \\
\text { mb-mb }\end{array}$ & $\begin{array}{l}E_{s}^{\exp } \\
\mathrm{MeV}\end{array}$ & $\chi^{2}$ & $\begin{array}{l}R_{g} \\
\text { fm }\end{array}$ & $\begin{array}{l}V_{g} \\
\mathrm{MeV}\end{array}$ & $\begin{array}{l}W_{g} \\
\mathrm{MeV}\end{array}$ & $\begin{array}{l}E_{s}^{g} \\
\mathrm{MeV}\end{array}$ & $\begin{array}{l}A_{0} \\
\mathrm{MeV}^{-1}\end{array}$ & $\begin{array}{l}B_{0} \\
\mathrm{MeV}^{1 / 2}\end{array}$ & $\begin{array}{l}E_{s}^{l} \\
\mathrm{MeV}\end{array}$ & Ref. \\
\hline${ }^{16} \mathrm{O}+{ }^{18} \mathrm{O}$ & 24.41 & EVR & 21 & $0.006-224$ & & 0.38 & 7.95 & 9.89 & 0.873 & & -3.13 & 141.6 & 6.54 & [87] \\
\hline${ }^{12} \mathrm{C}+{ }^{24} \mathrm{Mg}$ & 16.30 & EVR & 21 & $0.015-668$ & 9.7 & 2.54 & 6.40 & 11.10 & 0.741 & 9.87 & -6.32 & 297.2 & 9.89 & {$[174,216,217]$} \\
\hline${ }^{12} \mathrm{C}+{ }^{30} \mathrm{Si}$ & 14.11 & EVR & 22 & $0.0027-815$ & 10.5 & 1.08 & 7.79 & 13.31 & 0.962 & 10.54 & -4.33 & 274.1 & 10.74 & {$[141,218]$} \\
\hline${ }^{6} \mathrm{Li}+{ }^{198} \mathrm{Pt}$ & 8.53 & EVR & 10 & $0.00017-348$ & & 45.7 & 7.89 & 27.43 & 1.75 & & -3.14 & 545.5 & 19.27 & [69] \\
\hline${ }^{7} \mathrm{Li}+{ }^{198} \mathrm{Pt}$ & 8.82 & EVR & 11 & $0.0002-1004$ & & 5.93 & 9.71 & 28.04 & 1.65 & & -2.57 & 507.2 & 18.58 & [70] \\
\hline${ }^{24} \mathrm{Mg}+{ }^{30} \mathrm{Si}$ & 17.89 & EVR & 20 & $0.0080-332$ & 20.8 & 0.59 & 8.19 & 24.05 & 1.05 & 21.22 & -7.12 & 984.5 & 20.91 & {$[173,207]$} \\
\hline${ }^{28} \mathrm{Si}+{ }^{28} \mathrm{Si}$ & 10.92 & EVR & 21 & $0.00063-453$ & & 13.5 & 8.39 & 28.95 & 1.26 & 22.51 & -3.45 & 874.0 & 23.64 & [159] \\
\hline${ }^{28} \mathrm{Si}+{ }^{30} \mathrm{Si}$ & 14.30 & EVR & 17 & $0.0044-500$ & 24.7 & 0.97 & 7.92 & 28.13 & 1.16 & 24.69 & -8.13 & 1369 & 24.73 & {$[161]$} \\
\hline${ }^{30} \mathrm{Si}+{ }^{30} \mathrm{Si}$ & 15.61 & EVR & 20 & $0.0049-916$ & & 0.52 & 8.63 & 27.94 & 1.15 & 24.46 & -5.40 & 968.1 & 22.93 & [219] \\
\hline${ }^{27} \mathrm{Al}+{ }^{45} \mathrm{Sc}$ & 9.63 & EVR & 16 & $0.00031-596$ & 32.4 & 0.68 & 7.11 & 37.68 & 1.42 & 32.17 & -7.14 & 1848 & 32.01 & {$[172]$} \\
\hline${ }^{11} \mathrm{~B}+{ }^{197} \mathrm{Au}$ & -5.00 & $\mathrm{EVR}+\mathrm{FF}$ & 17 & $0.0003-770$ & 39.8 & 18.8 & 10.56 & 46.81 & 1.78 & 39.51 & -5.29 & 1607 & 39.9 & [206] \\
\hline${ }^{32} \mathrm{~S}+{ }^{48} \mathrm{Ca}$ & 7.66 & EVR & 21 & $0.0008-490$ & & 8.63 & 8.56 & 42.82 & 1.64 & 33.9 & -6.97 & 2160 & 35.38 & [169] \\
\hline${ }^{36} \mathrm{~S}+{ }^{48} \mathrm{Ca}$ & 7.55 & EVR & 25 & $0.0006-973$ & & 3.26 & 10.00 & 41.97 & 1.11 & 38.93 & -10.32 & 3129 & 37.93 & [165] \\
\hline${ }^{12} \mathrm{C}+{ }^{198} \mathrm{Pt}$ & -13.96 & $\mathrm{EVR}+\mathrm{FF}$ & 20 & $0.0001-670$ & 48.0 & 12.5 & 10.89 & 55.38 & 1.81 & 48.68 & -3.86 & 1232 & 48.0 & {$[70]$} \\
\hline${ }^{40} \mathrm{Ca}+{ }^{48} \mathrm{Ca}^{*}$ & 4.56 & EVR & 23 & $0.0013-463$ & & 6.30 & 8.31 & 51.86 & 1.64 & 43.88 & -6.22 & 2624 & 42.13 & [163] \\
\hline${ }^{48} \mathrm{Ca}+{ }^{48} \mathrm{Ca}^{*}$ & -2.99 & EVR & 27 & $0.0006-506$ & & 7.43 & 9.87 & 51.17 & 1.08 & 48.45 & -13.0 & 4741 & 47.54 & {$[166,167]$} \\
\hline${ }^{28} \mathrm{Si}+{ }^{64} \mathrm{Ni}^{*}$ & -1.79 & EVR & 16 & $0.00003-506$ & 45.6 & 0.58 & 8.05 & 50.45 & 1.40 & 45.73 & -10.28 & 3791 & 45.65 & {$[162]$} \\
\hline${ }^{36} \mathrm{~S}+{ }^{64} \mathrm{Ni}^{*}$ & -8.54 & EVR & 24 & $0.0028-788.9$ & 52.3 & 10.5 & 9.66 & 56.20 & 1.21 & 52.8 & -17.4 & 5993 & 53.1 & [168] \\
\hline${ }^{16} \mathrm{O}+{ }^{208} \mathrm{~Pb}^{*}$ & -46.48 & $\mathrm{EVR}+\mathrm{FF}$ & 38 & $0.000016-1133$ & 68.0 & 106 & 10.43 & 73.59 & 1.57 & 69.06 & -3.91 & 667.7 & 69.44 & {$[110,142]$} \\
\hline${ }^{28} \mathrm{Si}+{ }^{94} \mathrm{Mo}$ & 35.29 & EVR & 15 & $0.0039-381$ & & 3.58 & 6.99 & 73.17 & 1.43 & 69.18 & -5.04 & 1465 & & [220] \\
\hline${ }^{48} \mathrm{Ti}+{ }^{58} \mathrm{Fe}$ & -23.51 & EVR & 26 & $0.0018-351$ & & 0.49 & 8.07 & 70.99 & 1.71 & 63.13 & -5.08 & 1924.7 & 62.05 & [144] \\
\hline${ }^{54} \mathrm{Fe}+{ }^{58} \mathrm{Ni}$ & -5.40 & EVR & 25 & $0.0011-433$ & 86.7 & 24.2 & 9.13 & 91.51 & 1.76 & 84.78 & -3.05 & 761.9 & 83.05 & [46] \\
\hline${ }^{40} \mathrm{Ca}+{ }^{96} \mathrm{Zr}^{*}$ & -41.09 & EVR & 62 & $0.0027-474$ & & 5.67 & 9.63 & 94.15 & 2.95 & & -5.02 & 1476 & 78.17 & {$[146]$} \\
\hline${ }^{58} \mathrm{Ni}+{ }^{64} \mathrm{Ni}$ & -52.72 & EVR & 38 & $0.0013-308$ & & 2.40 & 6.70 & 94.74 & 2.22 & 80.96 & -5.04 & 1465 & 85.70 & [152] \\
\hline${ }^{64} \mathrm{Ni}+{ }^{64} \mathrm{Ni}^{*}$ & -48.80 & EVR & 16 & $0.00002-442$ & 87.5 & 0.71 & 8.86 & 92.62 & 1.45 & 87.7 & -8.29 & 2646 & 87.54 & {$[12]$} \\
\hline${ }^{37} \mathrm{Cl}+{ }^{130} \mathrm{Te}$ & -56.57 & EVR & 17 & $0.0012-648$ & & 0.50 & 11.15 & 102.1 & 2.15 & 87.7 & -.70 & 1305 & 91.26 & [154] \\
\hline${ }^{40} \mathrm{Ar}+{ }^{112} \mathrm{Sn}^{*}$ & -63.20 & $\mathrm{EVR}+\mathrm{FF}$ & 15 & $0.0084-478$ & & 4.16 & 9.19 & 104.40 & 2.45 & 87.70 & -3.41 & 990.8 & 87.54 & [221] \\
\hline${ }^{40} \mathrm{Ar}+{ }^{116} \mathrm{Sn}^{*}$ & -62.36 & $\mathrm{EVR}+\mathrm{FF}$ & 14 & $0.0038-512$ & & 9.75 & 8.99 & 103.84 & 2.44 & 87.38 & -3.65 & 1061 & 92.95 & [221] \\
\hline${ }^{40} \mathrm{Ar}+{ }^{122} \mathrm{Sn}^{*}$ & -58.64 & $\mathrm{EVR}+\mathrm{FF}$ & 17 & $0.0018-661$ & & 8.97 & 9.90 & 103.79 & 2.66 & 83.59 & -3.02 & 1023 & 89.75 & [221] \\
\hline${ }^{40} \mathrm{Ar}+{ }^{144} \mathrm{Sm}^{*}$ & -90.66 & $\mathrm{EVR}+\mathrm{FF}$ & 11 & $0.0016-322$ & & 6.85 & 8.20 & 124.49 & 2.29 & 111.46 & -1.96 & 489.8 & 113.3 & [221] \\
\hline${ }^{40} \mathrm{Ar}+{ }^{148} \mathrm{Sm}^{*}$ & -84.18 & $\mathrm{EVR}+\mathrm{FF}$ & 12 & $0.0008-353$ & & 7.28 & 9.16 & 125.60 & 3.51 & & -1.13 & 382.0 & 103.9 & [221] \\
\hline${ }^{40} \mathrm{Ar}+{ }^{154} \mathrm{Sm}^{*}$ & -75.31 & $\mathrm{EVR}+\mathrm{FF}$ & 15 & $0.0016-407$ & & 24.4 & 8.38 & 122.80 & 4.17 & & -1.51 & 528.1 & 98.28 & [221] \\
\hline${ }^{76} \mathrm{Ge}+{ }^{86} \mathrm{Kr}$ & -97.91 & EVR & 15 & $0.0068-347$ & & 14.6 & 8.08 & 129.95 & 2.94 & 98.51 & -1.94 & 394.3 & 116.3 & {$[222]$} \\
\hline${ }^{58} \mathrm{Ni}+{ }^{124} \mathrm{Sn}$ & -112.30 & $\mathrm{EVR}+\mathrm{FF}$ & 15 & $0.00046-570$ & & 1.05 & 8.51 & 156.92 & 3.45 & 115.84 & -2.18 & 6592 & 138.3 & {$[223,224]$} \\
\hline${ }^{64} \mathrm{Ni}+{ }^{124} \mathrm{Sn}$ & -117.51 & $\mathrm{EVR}+\mathrm{FF}$ & 17 & $0.0008-605$ & & 1.71 & 7.86 & 154.02 & 2.59 & 135.63 & -2.35 & 602.1 & 141.9 & {$[223,224]$} \\
\hline
\end{tabular}

a fraction extend to the very low cross section region. For medium- and heavy-mass systems, there are 35 such measurements ranging from ${ }^{16} \mathrm{O}+{ }^{18} \mathrm{O}$ to ${ }^{64} \mathrm{Ni}+{ }^{124} \mathrm{Sn}$ as summarized in Table 2. Among them are 13 systems which have been studied by Siwek-Wilczynska et al. [214]. These are indicated with a star in column 1 . The fusion $Q$ values, the measured reaction channels (fusion-evaporation and/or fusion-fission), the number of data points, and the cross sec- tion range are listed in columns $2-5$. We note that these 35 excitation functions all represent complete fusion. That is, when there are contributions from fission, the data are from the sum of measurements from fusion evaporation and fusion fission. Column 6 gives the energy of the S-factor maximum for those systems where a value has been determined in the experiments. 


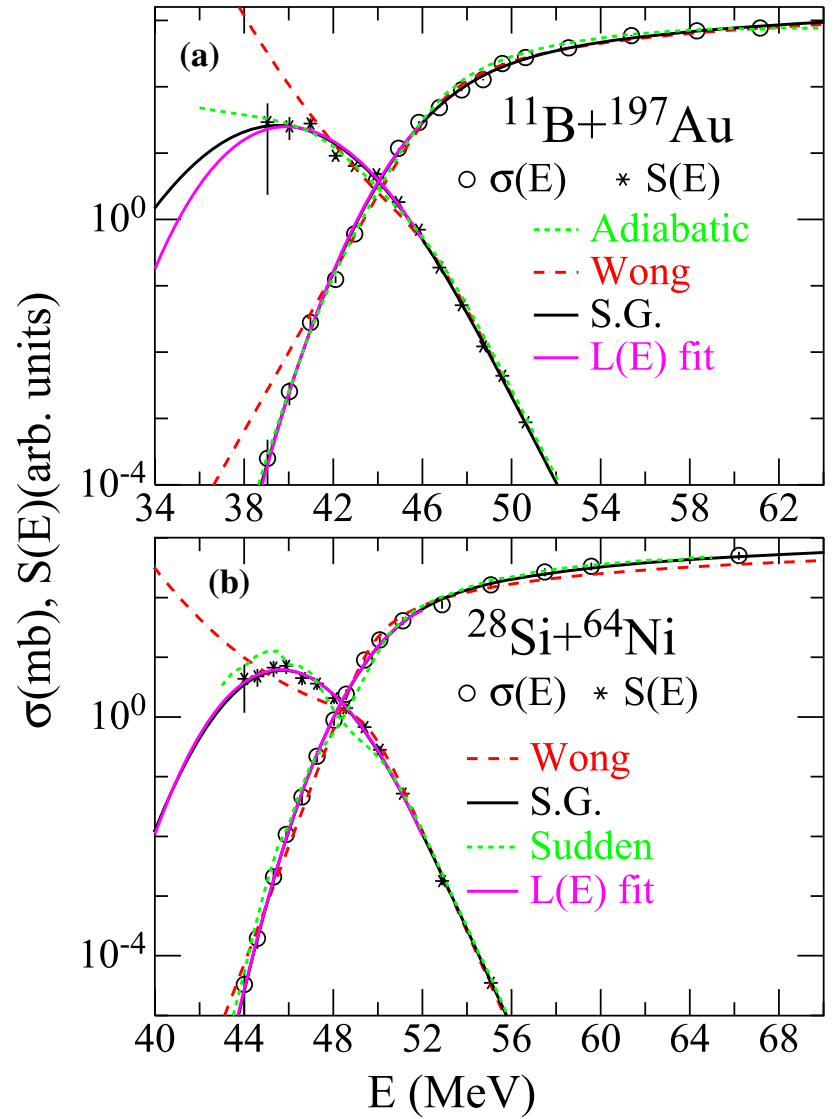

Fig. 56 Fusion cross sections, $\sigma(E)$ and the corresponding $S(E)$ factors for the systems ${ }^{11} \mathrm{~B}+{ }^{197} \mathrm{Au}$ [206] (panel a) and ${ }^{28} \mathrm{Si}+{ }^{64} \mathrm{Ni}$ [162] (panel b). See text for details

Columns 7-10 give the least-squares fit parameters, $\chi^{2}$, $R_{g}, V_{g}$, and $W_{g}$ obtained from the Gaussian formula Eq. 41 . The parameters $A_{0} B_{0}$ from the logarithmic derivative $L(E)$ fits (see Sect. 4), are listed in columns 12 and 13, respectively. The values $\chi^{2}$ are defined as:

$\chi^{2}=\frac{1}{N-M} \sum_{i=1}^{N}\left(\left(\sigma_{i}-\sigma_{\text {exp-i }}\right) / \Delta \sigma_{\exp -i}\right)^{2}$,

where $\sigma_{i}$ and $\sigma_{\exp -i}$ are calculated and experimental cross sections, respectively; $\Delta \sigma_{\text {exp }}$ are the experimental uncertainties, and $N, M$ are the number of experimental data points and adjustable parameters, respectively. For the Gaussian formula $M=3$.

Well-developed experimental $S$-factor maxima are seen in twelve systems for which the experimental values of $E_{s}^{\exp }$ are given in column 6 . Estimates of $E_{s}$ for most of the systems have also been obtained using the single-Gaussian test distribution to fit the excitation function or Eq. 25,26 to reproduce the logarithmic derivative. The values of $E_{S}$ obtained from the two extrapolation methods are very similar and in good agreement also with the $E_{s}^{\text {exp }}$ values derived directly

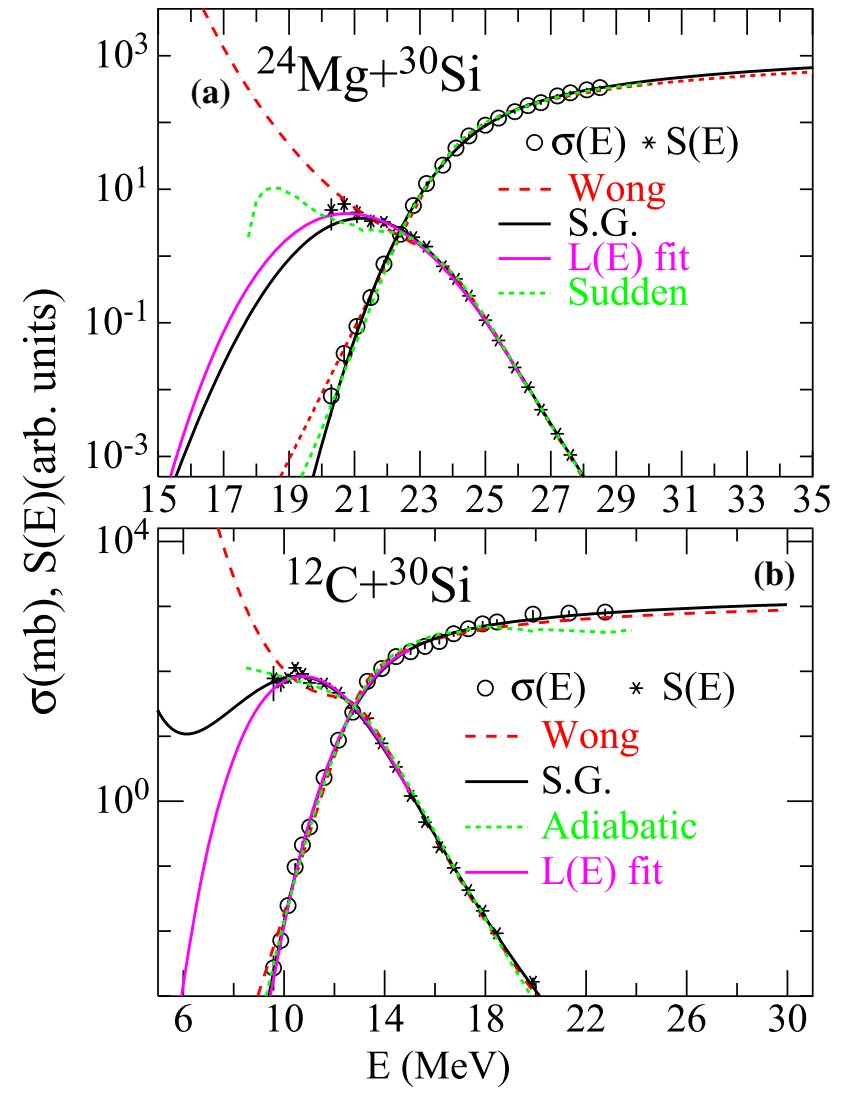

Fig. 57 Fusion cross sections, $\sigma(E)$ and the corresponding $S(E)$ factors for the systems ${ }^{24} \mathrm{Mg}+{ }^{30} \mathrm{Si}[173,207]$ (panel a) and ${ }^{12} \mathrm{C}+{ }^{30} \mathrm{Si}$ $[141,218]$ (panel b). See text for details

from the experimental data in the twelve systems mentioned above.

Four systems, two with negative fusion $Q$ values, ${ }^{11} \mathrm{~B}+{ }^{197}$ $\mathrm{Au}$ and ${ }^{28} \mathrm{Si}+{ }^{64} \mathrm{Ni}$, and two with positive fusion $Q$ values, ${ }^{24} \mathrm{Mg}+{ }^{30} \mathrm{Si}$ and ${ }^{12} \mathrm{C}+{ }^{30} \mathrm{Si}$, are shown in Figs. 56 and 57, respectively. Fusion cross sections are given by open circles while $S$ factors are shown by stars.

Several calculations are included in these figures. The black solid curves (S.G.) represent results from the singleGaussian distribution, while the magenta solid curves are derived from fits to the low energy $L(E)$ data in the energy region below the fusion barrier. Also included are the calculations from the Wong formula and CC calculations, using the sudden model $[123,124]$ or the adiabatic model $[126,127]$, as indicated. The inability of the Wong formula to reproduce the sharp drop-off of the cross sectioin at the lowest energies can be seen for all four systems.

By examining Table 2, Figs. 56 and 57, one observes that: (1) The two empirical fits, using either the Gaussian test distribution or the logarithmic-derivative fit reproduce the excitation function and the hindrance behavior at low energies rather well. For the low cross section measurements, there is no obvious preference for one approach over the other 


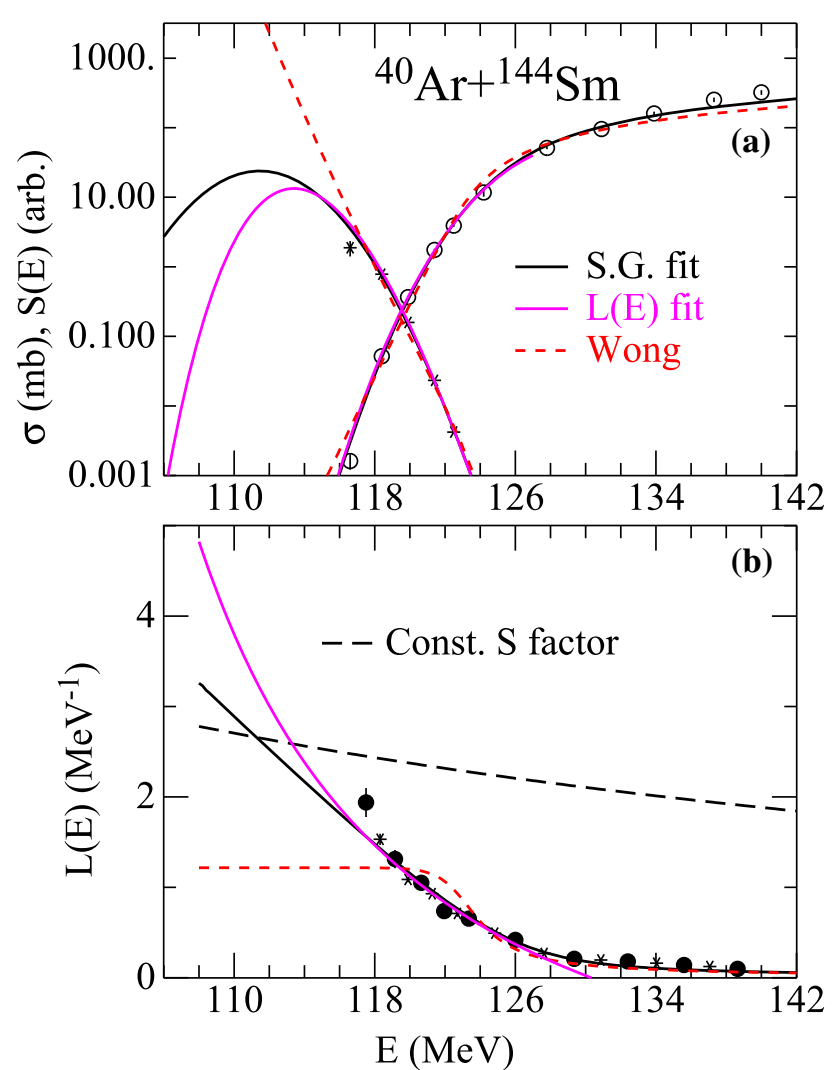

Fig. 58 Fusion cross sections, $\sigma(E)$ and the corresponding $S(E)$ factors, (panel a) and $L(E)$ (panel b) for the system ${ }^{40} \mathrm{Ar}+{ }^{144} \mathrm{Sm}[221]$

in terms of reproducing the hindrance behavior. (2) The $\chi^{2}$ values, which are obtained over the full energy range from the Gaussian distribution fits are better than the fit obtained by using the Wong formula, and they are often much better than what is obtained by Coupled-Channels calculations, although Coupled-Channels calculations are often not performed so detailed as a fitting process. (since the $L(E)$-data fit only deals with the cross sections at low energies, we do not calculate the $\chi^{2}$ value for that method), (3) The deviations between the predictions for $E_{S}$ from the two recipes are larger in cases where the measurements are less accurate. That can be seen from values of $E_{s}^{g}$ and $E_{s}^{l}$ listed in Table 2.

For comparison, plots for ${ }^{40} \mathrm{Ar}+{ }^{144} \mathrm{Sm}$ are shown in Fig. 58. In this case, the measured excitation function is far from reaching the energy region where the $S$-factor maximum is expected to appear. The extrapolations obtained by using the two recipes (black and magenta curves) both predict an $S$-factor maximum at a lower energy, consistent with the expectation that there must be an $S$-factor maximum since the fusion $Q$ value of ${ }^{40} \mathrm{Ar}+{ }^{144} \mathrm{Sm}$ is negative. However, there are very big differences in the predictions in the lower energy region from these two formulae due the shortage of measured data.
7.6 Systematics of the Gaussian distribution parameters

The systematics the parameters $A_{0}$ and $B_{0}$ were already discussed in Sect. 6.

The parameters $R_{g}, V_{g}$ and $W_{g}$, from the single-Gaussian test distribution analysis exhibit a relatively smooth behavior when plotted in Fig. 59 as functions of the Coulomb barrier, given by

$Z_{P} Z_{T} e^{2} /\left[1.44\left(A_{P}^{1 / 3}+A_{T}^{1 / 3}\right)\right](\mathrm{MeV})$.

Here $Z_{p}, A_{p}$ and $Z_{T}, A_{T}$ are the nuclear charge and mass numbers for the projectile and the target, respectively. The barrier radius parameters $R_{g}$ are shown in Fig. 59 panel a, normalized to the value

$1.44\left(A_{P}^{1 / 3}+A_{T}^{1 / 3}\right)(\mathrm{fm})$.

Figs. 59 panel b, panel c give the results of the average barrier height $V_{g}$ and the width parameter $W_{g}$ of the test distribution normalized to the respective Coulomb barrier, Eq. (45). These parameters follow a general trend with small superimposed fluctuations due to nuclear structure effects.

These systematics can be used to make first-order predictions of fusion cross sections for unmeasured systems as done e.g. for the system ${ }^{12} \mathrm{C}+{ }^{24} \mathrm{Mg}$ in Ref. [17]. An example of a predicted excitation function for fusion of ${ }^{12} \mathrm{C}+{ }^{24} \mathrm{Mg}$ was shown in Ref. [17] published in 2018. At that time, experimental measurements had been performed only from 250 $1200 \mathrm{mb}$ and $500-1000 \mathrm{mb}$ by Daneshvar et al. [216] and Gary et al. [217], respectively. These measurement ranges are insufficient to obtain values of the three parameters in Eq. (41).

The cross sections and $S$ factors for the systems ${ }^{16} \mathrm{O}+{ }^{18} \mathrm{O}$ [87] and ${ }^{12} \mathrm{C}+{ }^{30} \mathrm{Si}[141,218]$ had been measured and were shown in Fig. 6, panel a and c of Ref. [17]. The solid curves in panel a of that figure are the result of least-squares fits using Eq. (41) for ${ }^{12} \mathrm{C}+{ }^{30} \mathrm{Si}$ with parameters tabulated in Table 2. There is an indication of an $S$-factor maximum for ${ }^{12} \mathrm{C}+{ }^{30} \mathrm{Si}$ at a c.m. energy of about $11 \mathrm{MeV}$, while for ${ }^{16} \mathrm{O}+{ }^{18} \mathrm{O}$ no maximum in the $S$ factor can be seen in the measured energy range. The interpolated values for the ${ }^{12} \mathrm{C}+{ }^{24} \mathrm{Mg}$ system obtained in Ref. [17] are $R_{g}=7.88, V_{g}=11.50$ and $W_{g}=$ 0.91 , with predicted cross sections shown by the red dashed lines in panel $\mathrm{b}$ of that figure. As in the ${ }^{12} \mathrm{C}+{ }^{30} \mathrm{Si}$ case, a maximum of the $S$ factor is predicted to occur at a c.m. energy of about $9 \mathrm{MeV}$ in Ref. [17]. Figure 60, including the predictions based on the Gaussian distribution and the logarithmic derivative, is taken from Ref. [17].

Recently, the fusion excitation function for the system ${ }^{12} \mathrm{C}+{ }^{24} \mathrm{Mg}$ was measured at Legnaro National Laboratory (LNL) using inverse kinematics at low energies [141]. An $S$ factor maximum was seen with an energy of $E_{s}=9.7 \mathrm{MeV}$. The new measurements have been added in Fig. 60 panel b (black squares) together with the two fits (green and black 


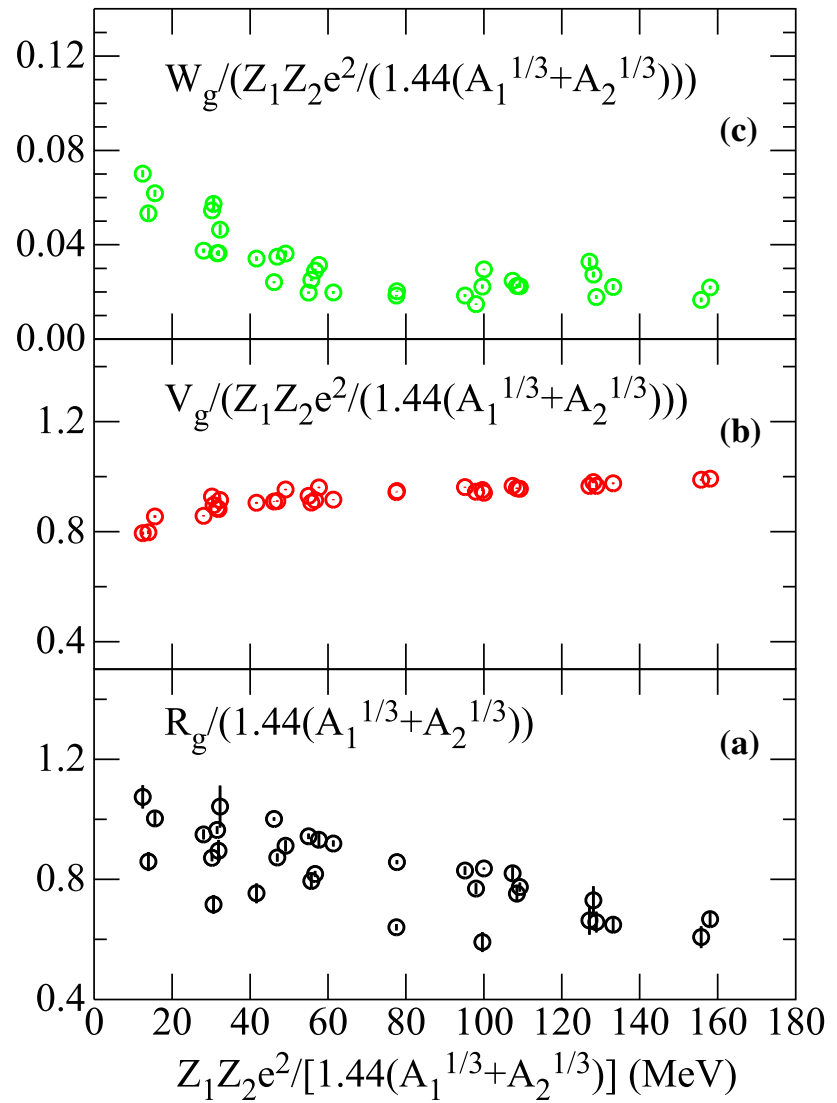

Fig. 59 Plots of the parameters $R_{g}, V_{g}$ and $W_{g}$ as function of the Coulomb barrier $Z_{1} Z_{2} e^{2} /\left[1.44\left(A_{1}^{1 / 3}+A_{2}^{1 / 3}\right)\right](\mathrm{MeV})$. In order to reduce the strong system-dependence, the parameters are scaled by factors $1.44\left(A_{1}^{1 / 3}+A_{2}^{1 / 3}\right)(\mathrm{fm})$ (Eq. (45)) and $Z_{1} Z_{2} e^{2} /\left[1.44\left(A_{1}^{1 / 3}+A_{2}^{1 / 3}\right)\right]$ $(\mathrm{MeV})($ Eq. (46)). See text for details

curves). Although there is a slight change in the Gaussian distribution fit which now exhibits an $S$-factor maximum at $E_{S}^{g}=9.87 \mathrm{MeV}$, it is clear that the prediction of Ref. [17] based on the data at higher energy and the systematics was quite accurate.

\subsection{Complications in studies of heavy systems}

In the light and medium-mass systems discussed above, there is little ambiguity about the identification of the complete fusion process by the detection of evaporation residues formed after the emission of a limited number of neutrons and charged particles and followed by $\gamma$ emission.

However, for reactions involving loosely-bound projectiles, the assumption of complete fusion implied for most of the systems discussed in this work does not hold. Sometimes a substantial fraction of the incoming flux leads to incomplete fusion reactions in which only a fraction of the projectile combines with the target nucleus while the rest escapes the nuclear attraction. Sophisticated coincidence experiments are needed to determine the full kinematics of this process

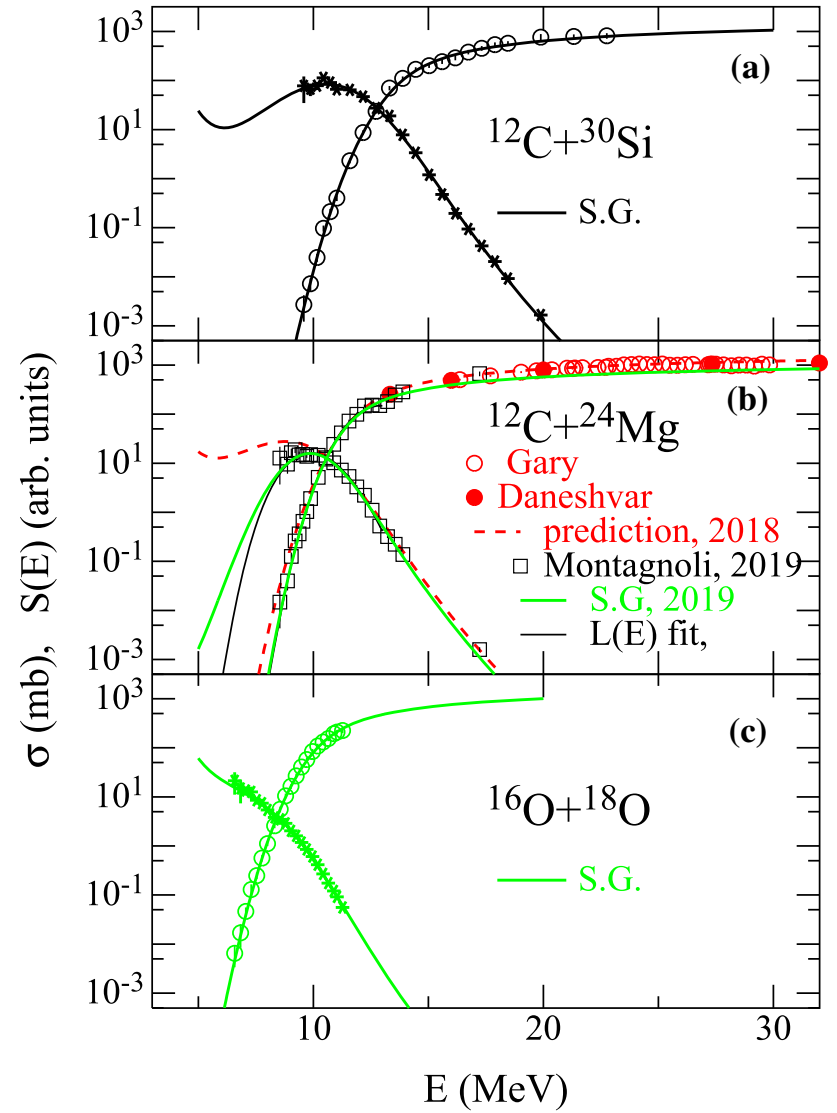

Fig. 60 A reproduction of Fig. 6 of Ref. [17]: comparison for cross sections and $S$ factors (arbitrary units) for fusion of ${ }^{12} \mathrm{C}+{ }^{30} \mathrm{Si}(\mathbf{a})$, ${ }^{12} \mathrm{C}+{ }^{24} \mathrm{Mg}(\mathbf{b})$ and ${ }^{16} \mathrm{O}+{ }^{18} \mathrm{O}(\mathbf{c})$. The red-dashed curves in $(\mathbf{b})$ are original predictions obtained from Ref. [17]. The black open square, green and white curves are the new measurements and two fits by including the new data [174]

in order to separate complete and incomplete fusion events. Recent studies addressing this situation are described in Refs. $[69,225]$. For reactions involving loosely bound projectiles, such as e.g. ${ }^{6,7} \mathrm{Li}$, one must therefore exercize caution in interpreting the measured evaporation residue excitation function. The ${ }^{7} \mathrm{Li}+{ }^{198} \mathrm{Pt}$ system, listed in Table 2 , is one such example [70].

Heavy systems also present complications in terms of identifying complete fusion events. In reactions that reach actinide and heavier systems, the fission decay branch can become competitive with the particle evaporation channels because of the reduction of the fission barrier when compared to lighter systems. For some systems, e.g. ${ }^{16} \mathrm{O}+{ }^{208} \mathrm{~Pb}$ [110], the complete fusion cross section is composed of an evaporation and a fusion-fission branch. In this case, both branches must be measured into the sub-barrier region and only the resulting sum of the cross-sections represents a reliable measurement of the complete fusion process. This is demonstrated for the ${ }^{16} \mathrm{O}+{ }^{208} \mathrm{~Pb}$ system in Fig. 61. Here the cross sections for fusion-evaporation (upward pointing 


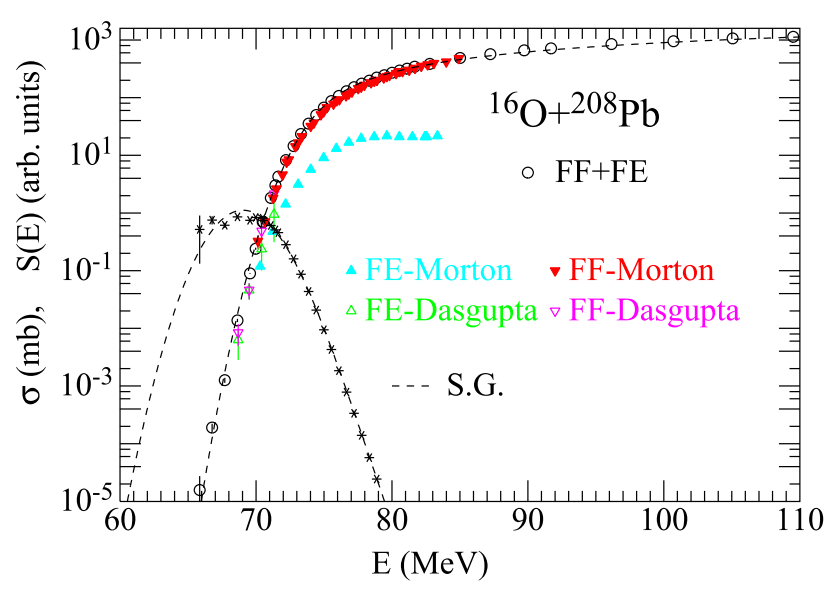

Fig. 61 Comparison of contributions from fusion evaporation (FE) and fusion fission (FF) for system ${ }^{16} \mathrm{O}+{ }^{208} \mathrm{~Pb}$ [110]. Open circles represent the total-fusion excitation function. Upward pointing triangles are from fusion evaporation, while downward triangles represent fusion fission. Stars represent the $S(E)$ factor, while the dashed curves are calculations coming from the single-Gaussian description

triangles) and fusion-fission (downward pointing triangles) are shown to contribute substantially to the total fusion cross section, especially below the center-of-mass energy of about $\sim 75 \mathrm{MeV}$.

The heaviest targets used in the reactions which are discussed in this article (see Table 2) are ${ }^{208} \mathrm{~Pb},{ }^{197} \mathrm{Au}$, and ${ }^{198} \mathrm{Pt}$. These, and neighboring nuclei, that may be populated via inelastic and few particle transfer reactions, have liquid-drop model fission barriers at low spins in the range of 13-17.5 MeV [226], which effectively suppresses the fission decay branch. For actinide targets with fission barriers in the 5-7 MeV range, transfer reactions can populate excitation energies above the fission barrier, and this sequential fission branch can become a substantial contamination to the complete fusion-fission cross section. Although the kinematics of fission fragments originating from these two fission processes provide some means of discrimination via the so-called folding angle between the fragments, this method of isolating fusion-fission events is not viable, especially at deep sub-barrier energies where cross sections are tiny. A clear discrimination would require measuring the charge, $\mathrm{Z}$, or mass, A, of both fission fragments. For a sufficiently heavy projectile, the deficit in total charge or mass of the fragments would signal that they came from a transfer-type reaction. This kind of experiment is, in principle, possible, but it has, to our knowledge, not been pursued to date.

In addition, a further complexity presents itself when using relative heavy projectiles incident onto heavy targets. For reaction partners with charge products $Z_{1} Z_{2}>1600$, it is well known that the quasi-fission process accounts for a substantial fraction of the total fission-like events. This type of reactions is most clearly recognized by a violation of the frag- ment mass and angle requirement for compound fission reactions, namely the forward-backward symmetry of the angular distribution for any fragment mass bin (see Refs. [227229]). The forward-backward symmetry signals a complete loss of memory of the incident orientation of the reaction partners as expected if a completely-fused compound nucleus was formed in the process. Although a fraction of the fission fragments underlying this mass-angle distribution may abide by the compound nucleus requirements, the two distributions are typically strongly overlapping such that no meaningful separation can be achieved. It has also been shown that even in cases where the mass-angle distributions seem to exhibit the compound-nucleus characteristics, excessive angular anisotropies [230] reveal the presence of a quasifission component.

Because of these aspects of heavy-ion reactions in very heavy systems, there are not many complete fusion data available covering a broad energy region, and our review for heavy systems is therefore rather restricted. The heaviest system included in Table 2 is ${ }^{64} \mathrm{Ni}+{ }^{124} \mathrm{Sn}$.

\section{Summary and outlook}

The process of heavy-ion fusion is a central theme in nuclear reaction studies. It represents a complicated rearrangement of nuclear matter from two individual nuclei into a single combined fused compound nucleus. The inverse process, nuclear fission, has of course played an even larger role over many decades, both in terms of fundamental science as well as in societal applications.

One may characterize the main focal points of the fusion studies as follows: (1) 1960's-1980, initial exploration of the heavy-ion fusion reaction; (2) 1980-1991, studies of the subbarrier enhancement caused by coupled-channels effects and an exploration of complete fusion limitations in very heavy systems; (3) 1991-2002, studies of the distributions of fusion barrier heights; (4) 2002-now, hindrance of the fusion cross section at extreme sub-barrier energies.

This review is focused mainly on the discovery and study of the deep subbarrier fusion hindrance, which was neither foreseen nor recognized previously.

In this article, we have reviewed various representations of the data which are useful in studying and deriving relevant parameters for the subbarrier hindrance effect. The $S$ factor representation is traditionally used in extrapolations of experimental data into the very low energy region relevant for stellar burning and explosions. However, it was for the first time introduced for heavier systems in the analyses of subbarrier fusion hindrance and it has become an essential analysis tool in the field. In addition, the logarithmic derivative, $L(E)$, of the energy-weighted cross section is also used in many cases to explore the hindrance effect. 
The measurement of the tiny fusion cross sections ( $\mu \mathrm{b}$ $\rightarrow \mathrm{pb}$ ) relevant for the present review poses severe experimental challenges. This experimental study requires new methods which have been developed at various laboratories as reviewed in Sect. 2.

The wealth of new fusion data, that appeared over the last decades, inspired the development of theoretical models and methods used to extract pertinent information about the reaction mechanism. The theoretical models have been discussed in Sect. 3. The models range from simple classical fusion (black disk) model, via the Wong model that includes the effects of quantum mechanical barrier tunneling to sophisticated coupled-channel models that include the effects of inelastic and transfer channels on the fusion cross section.

A consequence of the channel coupling is an enhancement of the cross section in the region around and below the static fusion barrier. It is naturally described by multiple barriers reflecting the perturbations to the classical barrier caused by various couplings. A large number of studies using this approach have been carried out and yielded a deeper understanding of the prevalent properties of the heavy-ion fusion process.

However, the discovery of deep subbarrier fusion hindrance in 2002 [11-13] showed that there was an aspect missing in the theory that manifested itself mainly at the hard-to-reach low-energy region. Although this effect was present in some of the earlier data, it had been missed in the analysis. As newer experiments confirmed the existence of this new phenomenon in many medium-mass systems, a modification of the coupled-channels theories was required. Two separate extensions of the coupled-channels formalism were proposed that appear to reproduce the observed effect. One, the sudden model, is based on the concept of a modification of the heavy-ion potential at distances where the matter distributions of the two nuclei overlap leading to a more shallow potential in this region $[123,124]$. The second proposed model includes an adiabatic readjustment of the coupling strength, which also appears to be able to reproduce the experimental results $[126,127]$. At present, the question of which model best captures the physics of the observed effect is not settled. One may hope that further theoretical and additional experimental work will be carried out in order to arrive at a deeper understanding of this phenomenon.

In Sect. 4, we reviewed the discovery and evidence for fusion hindrance, first seen in medium-heavy systems, but subsequently also identified in lighter systems toward those relevant for astrophysical systems. There, the influence on the hindrance behavior arising from various couplings is demonstrated, and it is shown that an $S$-factor maximum appears in systems as light as ${ }^{12} \mathrm{C}+{ }^{24} \mathrm{Mg}$ and ${ }^{12} \mathrm{C}+{ }^{30} \mathrm{Si}$.

As discussed in Sect. 5, it becomes an interesting question whether this effect is present also in lighter systems, such as those involved in astrophysical reaction networks. This is an important point, because measurements in the energy region relevant for these reactions are mostly impossible and one has to rely on various extrapolation procedures to estimate the reaction rate. Here we discuss the possibility of using extrapolation methods developed for heavier systems also in the lighter mass region. As shown, the difference can be quite substantial. The ${ }^{12} \mathrm{C}+{ }^{12} \mathrm{C}$ reaction is of special interest, but presently available data are not yet able to settle this issue in a satisfactory manner, partly because of the presense of resonances in this low-energy region. However, hints of an $S$ factor maximum at least in fusion of ${ }^{16} \mathrm{O}+{ }^{16} \mathrm{O}$ and ${ }^{12} \mathrm{C}+{ }^{16} \mathrm{O}$ have been found.

As fusion hindrance was found because the excitation functions cannot be described at low energies with standard CC calculations using the Woods Saxon potential, but later explained by the overlap of two colliding nuclei, a detailed understanding of the reaction dynamics at the overlap will be a main focus of future hindrance studies.

With the large number of reactions for which fusion cross sections have been measured into the deep subbarrier region, it is now possible to study the systematics of the fusion hindrance effect as presented in Sect. 6. The essential characteristic is the center-of-mass energy, $E_{S}$, at which the $S$ factor exhibits a maximum. This is summarized for many systems in Table 1. We also discuss another option for recognizing the subbarrier hindrance by comparing the data to coupled channels calculations performed under various assumptions.

The coupled-channels approach leads to a distribution of fusion barriers, which leaves an imprint on the fusion excitation function. As shown by Rowley et al. [10] this barrier distribution can be derived under a classical approximation directly from the data by a double differentiation of the product between energy and cross section.

An alternative approach, reviewed in Sect. 7, is to assume a specific test-function $D_{\text {test }}(V)$ representing the barrier distribution convoluted with the a barrier transmission function. From this function one can calculate the fusion excitation function for a comparison with the data. This approach was originally suggested by Stelson et al. [215], and subsequently used by Siwek-Wilczynska et al. [213,214] assuming a Gaussian distribution, which leads to a closed form expression for the fusion cross section

$$
\sigma=\frac{\sqrt{\pi} R^{2} W}{\sqrt{2} E}\left[\sqrt{\pi} Z \operatorname{erfc}(-Z)+\exp \left(-Z^{2}\right)\right] .
$$

It was found that for many systems this approach provides an excellent description of the cross section, even down into the deep subbarrier region. In fact, in many cases fits to the data using this method are very useful and comparable (or even better) to what can be achieved by the more sophisticated coupled-channels method. It thus appears that the tail of a Gaussian form of the convoluted distribution accurately represents the physics of the problem in the subbarrier region. We expect that this is caused by a sharper fall-off of the barrier 
transmission coefficient compared to those for standard ionion potentials used in coupled channels, as originally pointed out by Mişicu and Esbensen [123,124].

In Sect. 7, recent modifications of the Wong formula [27] have been reviewed. Rowley and Hagino [212] pointed out that the parameters in the Wong formula should be angular momentum dependent, and suggested to numerically derive the values of the relevant barrier parameters $V_{E}, R_{E}, \omega_{E}$. Alternatively, Denisov et al. [18] developed a recipe by introducing a collision-energy dependence of the barrier curvature in the Wong formula, which qualitatively reproduces the the hindrance behavior in a simple way.

In conclusion, the observation of fusion hindrance has in many ways revived the study of heavy-ion fusion reactions. Since the effect only appears at very low cross sections, it has given a strong incentive to pursue such measurements and develop improved techniques to reach this cross section level. Many aspects relating to the hindrance effect are still unsettled and further efforts in both experiments and theory are required in order to achieve a deeper understanding of this phenomenon. An important question is whether fusion hindrance plays a role in the light systems of astrophysical importance. This appears reasonable since the hindrance effect is related to the overlap of nuclear matter, which occurs in fusion reactions at extreme subbarrier energies, although it may have manifestations that are different from what has been observed in heavier systems. Some initial attempts to reach lower fusion cross sections, e.g. for ${ }^{12} \mathrm{C}+{ }^{12} \mathrm{C}$ as a crucial reaction in explosive scenarios for massive stars, have been undertaken using coincidence techniques $[56,62,65]$. Further developments along those lines are possible, but they require a determined and dedicated effort to make substantial progress.

Acknowledgements This work was supported by the US Department of Energy, Office of Nuclear Physics, under Contract No. DE-AC0206CH1 1357 for C. L. Jiang, K. E. Rehm and B. B. Back, by the European Union Seventh Framework Program FP7/2007-2013 under Grant Agreement No. 262010-ENSAR for A. M. Stefanini and G. Montagnoli, and by the ISPS KAKENHI Grant number JP19K03861 for K. Hagino.

Data Availability Statement This manuscript has no associated data or the data will not be deposited. [Authors' comment: No data will be deposited because this is a review paper that include only published results. Any new data generated by analysis of published data are provided in tabular or graphical form in the manuscript.]

Open Access This article is licensed under a Creative Commons Attribution 4.0 International License, which permits use, sharing, adaptation, distribution and reproduction in any medium or format, as long as you give appropriate credit to the original author(s) and the source, provide a link to the Creative Commons licence, and indicate if changes were made. The images or other third party material in this article are included in the article's Creative Commons licence, unless indicated otherwise in a credit line to the material. If material is not included in the article's Creative Commons licence and your intended use is not permitted by statutory regulation or exceeds the permitted use, you will need to obtain permission directly from the copyright holder. To view a copy of this licence, visit http://creativecomm ons.org/licenses/by/4.0/.

\section{References}

1. V.I. Zagrebaev, A.S. Denikin, A.V. Karpov, A.P. Alekseev, M.A. Naumenko, V.A. Rachkov, V.V. Samarin, V.V. Saiko, NRV web knowledge base on low-energy nuclear physics, http://nrv.jinr.ru/. Web site accessed during 2020

2. M. Dasgupta, D.J. Hinde, N. Rowley, A.M. Stefanini, Ann. Rev. Nucl. Part. Sci. 48, 401 (1998)

3. A.B. Balantekin, N. Takigawa, Rev. Mod. Phys. 70, 77 (1998)

4. K. Hagino, N. Takigawa, Prog. Theo. Phys. 128, 1061 (2012)

5. B.B. Back, H. Esbensen, C.L. Jiang, K.E. Rehm, Rev. Mod. Phys. 86, 317 (2014)

6. G. Montagnoli, A.M. Stefanini, Eur. Phys. J. A 53, 169 (2017)

7. C.H. Dasso, S. Landowne, A. Winther, Nucl. Phys. A 405, 381 (1983)

8. C.H. Dasso, S. Landowne, A. Winther, Nucl. Phys. A 407, 221 (1983)

9. J.X. Wei et al., Phys. Rev. Lett. 67, 3368 (1991)

10. N. Rowley, G.R. Satchler, P.H. Stelson, Phys. Lett. B 254, 25 (1991)

11. C.L. Jiang et al., Phys. Rev. Lett. 89, 052701 (2002)

12. C.L. Jiang et al., Phys. Rev. Lett. 93, 012701 (2004)

13. C.L. Jiang, H. Esbensen, B.B. Back, R.V.F. Janssens, K.E. Rehm, Phys. Rev. C 69, 014604 (2004)

14. C.A. Barnes, Adv. Nucl. Phys. 4, 133 (1968)

15. C.A. Barnes, S. Trentalange, S.C. Wu, Treatise on Heavy-Ion Science, by edited D.A. Bromley, vol. 6 (Plenum, New York, 1985), p. 3

16. M. Beckerman et al., Phys. Rev. Lett. 45, 1472 (1980)

17. C.L. Jiang, K.E. Rehm, B.B. Back, Eur. Phys. J. A 54, 218 (2018)

18. I.Yu. Denisov, I.Yu. Sedykh, Eur. Phys. J. A 54, 218 (2019)

19. FUSION97, Cairns, Queensland, Australia, J. Phys. G 23, 1157 (1997)

20. FUSION03, Matsushima. Miyagi, Japan, Prog. Theor. Phys. Suppl. 154, 1 (2004)

21. FUSION06, Venice, Italy, AIP Conf. Ser. 853, 1 (2006)

22. FUSION08, Chicago, USA, AIP Conf. Ser. 1098, 1 (2008)

23. FUSION11, Saint Malo, France, EPJ Web Conf. 17, 00001 (2001)

24. FUSION14, New Delhi, India, EPJ Web of Conf. 86, 00001 (2015)

25. FUSION17, Hobart, Tasmania, Australia, EPJ Web Conf. 163, $00001(2017)$

26. J.M. Blatt, V.F. Weisskopf, Theoretical Nuclear Physics (Wiley, New York, 1952), p. 346

27. C.Y. Wong, Phys. Rev. Lett. 31, 766 (1973)

28. K.E. Rehm, H. Esbensen, C.L. Jiang, B.B. Back, A.M. Stefanini, G. Montagnoli, Phys. Rev. C 94, 044612 (2016)

29. E. Margaret Burbidge, G.R. Burbidge, A.William Fowler, F. Hoyle, Rev. Mod. Phys. 29, 547 (1957)

30. A.B. Balantekin, S.E. Koonin, J.W. Negele, Phys. Rev. C 28, 1565 (1983)

31. R. Vandenbosch, Annu. Rev. Nucl. Sci. Part. 42, 447 (1992)

32. M. Beckerman et al., Phys. Rev. C 25, 837 (1982)

33. J.G. Keller, K.-H. Schmidt, F.P. He, G. Münzenberg, W. Reisdorf, H.-G. Clerc, C.-C. Sahm, Nucl. Phys. A 452, 173 (1986)

34. R. Bass, Nuclear Reactions with Heavy Ions (Springer, Berlin, 1980)

35. RST75.4934, Reports of Argonne National Laboratory (2004)

36. C.L. Jiang et al., Fusion excitation of ${ }^{58} \mathrm{Ni}+{ }^{89} \mathrm{Y}$, Annual Report of Phys. Div., Argonne National laboratory (2002) 
37. T. Spillane et al., Phys. Rev. Lett 98, 122501 (2007)

38. C.N. Davids, J.D. Larson, Nucl. Instr. Meth. Phys. Res. B 40-41, 1224 (1989)

39. C.L. Jiang et al., Nucl. Instr. Meth. Phys. Res. A 554, 500 (2005)

40. S. Hofmann, G. Münzenberg, Rev. Mod. Phys. 72, 733 (2000)

41. W. Reisdorf et al., Phys. Rev. Lett. 49, 1811 (1982)

42. G. Münzenberg, W. Faust, S. Hofmann, P. Armbruster, K. Güttner, H. Ewald, Nucl. Instr. Meth. 161, 65 (1979)

43. SHIP separator at GSI, Authors unknown

44. D.J. Hinde, M. Dasgupta, J.R. Leigh, J.C. Mein, C.R. Morton, J.O. Newton, H. Timmers, Phys. Rev. C 53, 1290 (1996)

45. N. Dasgupta, International on Meeting "Selected Topics in Nuclear and Atomic Physics", Fiera di Primiero (Trento, Italy), Sept. 30-Oct. 4, 2007, unpublished

46. A.M. Stefanini et al., Phys. Rev. C 82, 014614 (2010)

47. M.D. Rodríguez et al., Nucl. Instr. Meth. Phys. Res. A 614, 119 (2010)

48. P.H. Stelson, H.J. Kim, M. Beckerman, D. Shapira, R.L. Robinson, Phys. Rev. C 41, 1584 (1990)

49. J.F. Liang et al., Phys. Rev. C 94, 024616 (2016)

50. D. Shapira, J.F. Liang, C.J. Gross, R.L. Varner, H. Amro, C. Harlin, J.J. Kolata, S. Novotny, Nucl. Instr. Meth. Phys. Res. A 551, $330(2005)$

51. S. Beghini, C. Signorini, S. Lunardi, M. Morando, G. Fortuna, A.M. Stefanini, W. Meczynski, R. Pengo, Nucl. Instr. Meth. Phys. Res. A 239, 585 (1985)

52. H.Q. Zhang et al., Chin. Phys. C 34, 1628 (2010)

53. H.Q. Zhang et al., Phys. Rev. C 82, 054609 (2010)

54. H.M. Jia et al., Phys. Rev. C 86, 044621 (2012)

55. C.L. Jiang et al., Nucl. Instr. Meth. Phys. Res. A 682, 12 (2012)

56. C.L. Jiang et al., Phys. Rev. C 97, 012801(R) (2018)

57. N. Madhavan et al., Heavy Reaction Reaction Analyzer, : https:// www.iuac.res.in/heavy-ion-reaction-analyzer, accessed March 2021

58. J. Zickefoose et al., Phys. Rev. C 97, 065806 (2018)

59. Universita' degli Studi della Campania, Dipartimento di Matematica e Fisica, author unknown, https://www.circe.unicampania. it/, accessed March 2021

60. I.Y. Lee, Nucl. Phys. A 520, 641c (1990)

61. X. Fang et al., Phys. Rev. C 96, 045804 (2017)

62. W. Tan et al., Phys. Rev. Lett. C 124, 192702 (2020)

63. M. Heine et al., Nucl. Instr. Meth. Phys. Res. A 903, 1 (2018)

64. R. Shearman et al., Rad. Phys. Chem. 140, 475 (2017)

65. G. Fruet et al., Phys. Rev. Lett. 124, 192701 (2020)

66. I. Burducea, M. Straticiuc, D. Ghita, D. Mosu, C. Calinescu, N. Podaru, D. Mous, I. Ursu, N. Zamfir, Nucl. Instr. Meth. Phys. Res. B 359, 12 (2015)

67. R. Margineanu, C. Simion, S. Bercea, O. Duliu, D. Gheorghiu, A. Stochioiu, M. Matei, Appl. Radiat. Isot. 66, 1501 (2008)

68. N.T. Zhang et al., Phys. Lett. B 801, 135170 (2020)

69. A. Shrivastava et al., Phys. Rev. Lett. 103, 232702 (2009)

70. A. Shrivastava et al., Phys. Lett. B 755, 332 (2016)

71. A. Lemasson et al., Nucl. Instr. Meth. A 598, 445 (2009)

72. D. Seweryniak et al., Argonne Gas-Filled Analyzer, https://www. anl.gov/phy/argonne-gasfilled-analyzer, accessed March 2021

73. N. Madhavan et al., EPJ Web Conf. 17, 14003 (2011)

74. K. Sudarshan et al., Phys. Rev. C 95, 024604 (2017)

75. E. Prasad et al., Phys. Rev. C 84, 064606 (2011)

76. Varinderjit Singh et al., Phys. Rev. C 89, 024609 (2014)

77. G. Mohanto et al., Phys. Rev. C 88, 034606 (2013)

78. R.E. Tribble, C.A. Bertulani, M. La Cognata, A.M. Mukhamedzhanov, C. Spitaleri, Rep. Prog. Phys. 77, 106901 (2014)

79. A.M. Mukhamedzhanov, A.S. Kadyrov, D.Y. Pang, Eur. Phys. J. A 56, $233(2020)$

80. A. Tumino et al., Nature 557, 687 (2018)
81. L.C. Vaz, J.M. Alexander, G.R. Satchler, Phys. Rep. 69, 373 (1981)

82. R. Bass, Phys. Rev. Lett. 39, 265 (1977)

83. L.F. Canto, M.S. Hussein, Scattering Theory of Molecules, Atoms, and Nuclei (World Scientific, Singapore, 2013)

84. P.R. Christensen, Z.E. Switkowski, Nucl. Phys. A 280, 205 (1977)

85. S. Landowne, S.C. Pieper, Phys. Rev. C 29, 1352 (1984)

86. K. Hagino, N. Rowley, A.T. Kruppa, Comp. Phys. Commun. 123, 143 (1999)

87. J. Thomas et al., Phys. Rev. C 33, 1679 (1986)

88. G. Michaud, W.A. Fowler, Phys. Rev. C 2, 2041 (1970)

89. W.A. Fowler, G.R. Caughlan, B.A. Zimmerman, Ann. Rev. Astron. Astrophys. 13, 69 (1975)

90. R.A. Dayras, R.G. Stokstad, Z.E. Switkowski, R.M. Wieland, Nucl. Phys. A 265, 153 (1976)

91. X.D. Tang et al., J. Phys. Conf. Ser. 337, 012016 (2012)

92. N.T. Zhang et al., EPJ Web Conf. 109, 09003 (2016)

93. R. Ogura, K. Hagino, C.A. Bertulani, Phys. Rev. C 99, 065808 (2019)

94. Z.E. Switkowski, R.G. Stokstad, R.M. Wieland, Nucl. Phys. A 279, 502 (1977)

95. J.R. Leigh, M. Dasgupta, D.J. Hinde, J.C. Mein, J.X. Wei, N. Rowley, Phys. Rev. C 52, 3151 (1995)

96. G.R. Satchler, W.G. Love, Phys. Rep. 55, 183 (1979)

97. M.E. Brandan, G.R. Satchler, Phys. Rev. 285, 143 (1997)

98. D.T. Khoa, G.R. Satchler, Nucl. Phys. A 668, 3 (2000)

99. L.C. Chamon et al., Phys. Rev. C 66, 014610 (2002)

100. A.S. Umar, V.E. Oberacker, Phys. Rev. C 74, 021601(R) (2006)

101. A.S. Umar, C. Simenel, V.E. Oberacker, Phys. Rev. C 89, 034611 (2014)

102. K. Godbey, C. Simenel, A.S. Umar, Phys. Rev. C 100, 024619 (2019)

103. C.L. Jiang, B.B. Back, H. Esbensen, R.V.F. Janssens, K.E. Rehm, R.J. Charity, Phys. Rev. Lett. 110, 072701 (2013)

104. L.F. Canto, R. Donangelo, M.S. Hussein, P. Lotti, J. Lubian, J. Rangel, Phys. Rev. C 98, 044617 (2018)

105. H.-J. Fink, W. Scheid, W. Greiner, Nucl. Phys. A 188, 259 (1972)

106. G. Helling, W. Scheid, W. Greiner, Phys. Lett. B 36, 64 (1971)

107. J.M. Quesada, M. Lozano, G. Madurga, Phys. Lett. B 125, 14 (1983)

108. M.V. Andres, J.M. Quesada, M. Lozano, G. Madurga, Nucl. Phys. A 443, 380 (1985)

109. K. Hagino, N. Rowley, J. Phys. Conf. Ser. 590, 012020 (2015)

110. M. Dasgupta, D.J. Hinde, A. Diaz-Torres, B. Bouriquet, Catherine I. Low, G.J. Milburn, J.O. Newton, Phys. Rev. Lett. 99, 192701 (2007)

111. A. Diaz-Torres, D.J. Hinde, M. Dasgupta, G.J. Milburn, J.A. Tostevin, Phys. Rev. C 78, 064604 (2008)

112. J.M. Yao, K. Hagino, Phys. Rev. C 94, 011303(R) (2016)

113. Y. Kondo, T. Matsuse, Y. Abe, Prog. Theo. Phys. 59, 465 (1978)

114. B. Imanishi, Phys. Lett. B 27, 267 (1968)

115. B. Imanishi, Nucl. Phys. A 125, 33 (1969)

116. W. Scheid, W. Greiner, R. Lemmer, Phys. Rev. Lett. 25, 176 (1970)

117. C. Chin, R. Grimm, P. Julienne, E. Tiesinga, Rev. Mod. Phys. 82, $1225(2010)$

118. P. Naidon, S. Endo, Rep. Prog. Phys. 80, 056001 (2017)

119. M. Assuncao, P. Descouvemont, Phys. Lett. B 723, 355 (2013)

120. H. Esbensen, X. Tang, C.L. Jiang, Phys. Rev. C 84, 064613 (2011)

121. D.M. Brink, Prog. Theor. Phys. Suppl. 154, 268 (2004)

122. C.H. Dasso, G. Pollarolo, Phys. Rev. C 68, 054604 (2003)

123. S. Mişicu, H. Esbensen, Phys. Rev. Lett. 96, 112701 (2006)

124. S. Mişicu, H. Esbensen, Phys. Rev. C 75, 034606 (2007)

125. C. Simenel, A.S. Umar, K. Godbey, M. Dasgupta, D.J. Hinde, Phys. Rev. C 95, 031601(R) (2017)

126. T. Ichikawa, K. Hagino, A. Iwamoto, Phys. Rev. C 75, 057603 (2007) 
127. T. Ichikawa, K. Hagino, A. Iwamoto, Phys. Rev. Lett. 103, 202701 (2009)

128. T. Ichikawa, Phys. Rev. C 92, 064604 (2015)

129. T. Ichikawa, K. Matsuyanagi, Phys. Rev. C 88, 011602 (2013)

130. T. Ichikawa, K. Matsuyanagi, Phys. Rev. C 92, 021602(R) (2015)

131. K. Cheng, C. Xu, Phys. Rev. C 102, 014619 (2020)

132. K. Cheng, C. Xu, Phys. Rev. C 99, 014607 (2019)

133. T. Ichikawa, K. Hagino, A. Iwamoto, Phys. Rev. C 75, 064612 (2007)

134. S. Ohkubo, Phys. Rev. C 95, 044002 (2017)

135. K. Aoki, H. Horiuchi, Prog. Theo. Phys. 68, 1658 (1982)

136. K. Aoki, H. Horiuchi, Prog. Theo. Phys. 68, 2028 (1982)

137. K. Aoki, H. Horiuchi, Prog. Theo. Phys. 69, 857 (1983)

138. K. Aoki, H. Horiuchi, Prog. Theo. Phys. 69, 1154 (1983)

139. D. Baye, Phys. Rev. Lett. 58, 2738 (1987)

140. H. Fiedeldey et al., Phys. Rev. C 42, 411 (1990)

141. G. Montagnoli et al., Phys. Rev. C 97, 024610 (2018)

142. C.R. Morton, A.C. Berriman, M. Dasgupta, D.J. Hinde, J.O. Newton, K. Hagino, I.J. Thompson, Phys. Rev. C 60, 044608 (1999)

143. C.L. Jiang et al., Phys. Rev. C 71, 044613 (2005)

144. A.M. Stefanini et al., Phys. Rev. C 92, 064607 (2015)

145. R.A. Broglia, C.H. Dasso, S. Landowne, A. Winther, Phys. Rev. C 27, 2433 (1983)

146. A.M. Stefanini et al., Phys. Lett. B 728, 639 (2014)

147. H. Timmers, L. Corradi, A.M. Stefanini, D. Ackermann, J.H. He, S. Beghini, G. Montagnoli, F. Scarlassara, G.F. Segato, N. Rowley, Phys. Lett. B 399, 35 (1997)

148. H. Timmers, L. Corradi, A.M. Stefanini, D. Ackermann, J.H. He, S. Beghini, G. Montagnoli, F. Scarlassara, G.F. Segato, N. Rowley, Nucl. Phys. A 633, 421 (1998)

149. A.M. Stefanini et al., Phys. Rev. C 73, 034606 (2006)

150. Ö. Akyüz, Å. Winther, Nuclear Structure and Heavy-Ion Physics. In Proc. Int. School of Physics "Enrico Fermi", Course LXXVII, Varenna, edited by R.A.Broglia and R.A.Ricci (North Holland, Amsterdam, 1981)

151. H. Esbensen, G. Montagnoli, A.M. Stefanini, Phys. Rev. C 93, 034609 (2016)

152. A.M. Stefanini et al., Phys. Rev. C 100, 044619 (2019)

153. Kaixuan Cheng, Xu Chang, Phys. Rev. C 102, 014619 (2020)

154. R.N. Sahoo et al., Phys. Rev. C 102, 024607 (2020)

155. R.N. Sahoo et al., Phys. Rev. C 99, 024607 (2019)

156. C.L. Jiang, K.E. Rehm, B.B. Back, H. Esbensen, R.V.F. Janssens, A.M. Stefanni, G. Montagnoli, Phys. Rev. C 89, 051603(R) (2014)

157. A.M. Stefanini et al., Eur. Phys. J. A 49, 63 (2013)

158. C.L. Jiang et al., Phys. Rev. C 73, 14613 (2006)

159. G. Montagnoli et al., Phys. Rev. C 90, 044608 (2014)

160. G. Colucci et al., Phys. Rev. C 97, 044613 (2018)

161. C.L. Jiang et al., Phys. Rev. C 78, 017601 (2008)

162. C.L. Jiang et al., Phys. Lett. B 640, 18 (2006)

163. C.L. Jiang et al., Phys. Rev. C 82, 041601(R) (2010)

164. A. Mukherjee, M. Dasgupta, D.J. Hinde, K. Hagino, J.R. Leigh, J.C. Mein, C.R. Morton, J.O. Newton, H. Timmers, Phys. Rev. C 66, 034607 (2002)

165. A.M. Stefanini et al., Phys. Rev. C 78, 044607 (2008)

166. A.M. Stefanini et al., Phys. Lett. B 679, 95 (2009)

167. G. Montagnoli et al., Phys. Rev. C 85, 024607 (2012)

168. G. Montagnoli, A.M. Stefanini, L. Corradi, S. Courtin, E. Fioretto, F. Haas, D. Lebhertz, F. Scarlassara, R. Silvestri, S. Szilner, Phys. Rev. C 82, 064609 (2010)

169. G. Montagnoli et al., Phys. Rev. C 87, 014611 (2013)

170. C.L. Jiang, K.E. Rehm, B.B. Back, R.V.F. Janssens, Phys. Rev. C 75, 015803 (2007)

171. H. Esbensen, C.L. Jiang, Phys. Rev. C 79, 064619 (2009)

172. C.L. Jiang et al., Phys. Rev. C 81, 024611 (2010)

173. C.L. Jiang et al., Phys. Rev. Lett. 113, 022701 (2014)

174. G. Montagnoli et al., Phys. Rev. C 101, 044608 (2020)
175. C.L. Jiang, K.E. Rehm, B.B. Back, R.V.F. Janssens, Phys. Rev. C 79, 044601 (2009)

176. L.R. Gasques et al., Phys. Rev. C 76, 035802 (2007)

177. M.D. High, B. Cujec, Nucl. Phys. A 259, 513 (1976)

178. J. Zickefoose, Thesis, University of Connecticut; Doctoral Dissertation AA13485448 (2011)

179. B. Bucher et al., Phys. Rev. Lett. 114, 251102 (2015)

180. G. Hulke et al., Z. Phys. A 297, 161 (1980)

181. S.C. Wu et al., Nucl. Phys. A 422, 373 (1984)

182. S.C. Wu, Ph. D. Thesis, California Institute of Technology (1978)

183. H. Spinka et al., Nucl. Phys. A 233, 456 (1974)

184. J. Thomas et al., Phys. Rev. C 31, 1980 (1985)

185. A. Kuronen, J. Keinonen, P. Tikkanen, Phys. Rev. C 35, 591 (1987)

186. J.G. Duarte et al., J. Phys. G 42, 065102 (2015)

187. J.R. Patterson et al., Nucl. Phys. A 165, 545 (1971)

188. B. C̃ujec, C.A. Barnes, Nucl. Phys. A 266, 451 (1976)

189. B. Dasmahapatra, B. C̃ujec, F. Lahlou, Nucl. Phys. A 394, 301 (1983)

190. R.G. Stokstad et al., Phys. Rev. Lett. 37, 888 (1976)

191. M. Notani et al., Phys. Rev. C 85, 014607 (2012)

192. J.R. Patterson, H. Winkler, C.S. Zaidins, Astrophys. J. 157, 367 (1969)

193. M. Mazarakis, W.E. Stephens, Phys. Rev. C 7, 1280 (1973)

194. M.D. High, B. Cujec, Nucl. Phys. A 282, 181 (1977)

195. L. Barron et al., Nucl. Phys. A 779, 318 (2006)

196. E.E. Aguilera et al., Phys. Rev. C 73, 064601 (2006)

197. K.U. Kettner, H. Lorentz-Wirzba, C. Rolfs, Z. Phys. A 298, 65 (1980)

198. H.W. Becker, K.U. Kettner, C. Rolfs, H.P. Trautvetter, Z. Phys. A 303, 305 (1981)

199. A.M. Mukhamedzhanov, D.Y. Pang, A.S. Kadyrov, Phys. Rev. C 99, 064618 (2019)

200. C. Beck, A.M. Mukhamedzhanov, X. Tang, Eur. Phys. J. A 56, 87 (2020)

201. M. Beckerman, Nucl. Phys. A 278, 333 (1977)

202. A.S. Iljinov, M.V. Mebel, E. De Sanctis, C. Guaraido, V. Lucherini, V. Muccifora, E. Polii, A.R. Reolon, P. Rossi, Nucl. Phys. A 543, 517 (1992)

203. R. Perez-Torres, T.L. Belyaeva, E.F. Aguilera, Phys. Atomic Nuclei 59(8), 1372-1382 (2006). ISSN 1063-7788

204. R.L. Cooper, A.W. Steiner, E.F. Brown, Astro. Phys. J. 702(1), $660(2009)$

205. D.J. Hinde, A.C. Berriman, M. Dasgupta, J.R. Leigh, J.C. Mein, C.R. Morton, J.O. Newton, Phys. Rev. C 60, 054602 (1999)

206. A. Shrivastava et al., Phys. Rev. C 96, 034620 (2017)

207. A. Morsad, J.J. Kolata, R.J. Tighe, X.J. Kong, Phys. Rev. C 41, $988(1990)$

208. Z.E. Switkowski, R.G. Stokstad, R.M. Wieland, Nucl. Phys. A 279, 502 (1977)

209. Z.E. Switkowski, R.G. Stokstad, R.M. Wieland, Nucl. Phys. A 274, 202 (1976)

210. B. Dasmahapatra, B. Cujec, F. Lahlou, Can. J. Phys. 61, 657 (1983)

211. C.L. Jiang et al., Phys. Rev. C 75, 057604 (2007)

212. N. Rowley, K. Hagino, Phys. Rev. C 91, 044617 (2015)

213. K. Siwek-Wilczynska, E. Siemaszko, J. Wilczynski, Acta Phys. Polonica B 33, 451 (2002)

214. K. Siwek-Wilczynska, J. Wilczynski, Phys. Rev. C 69, 024611 (2004)

215. D.H. Stelson et al., Phys. Lett. 205, 190 (1987)

216. K. Daneshvar et al., Phys. Rev. C 25, 1342 (1982)

217. S. Gary, C. Volant, Phys. Rev. C 25, 1877 (1982)

218. W.J. Jordan, J.V. Maher, J. Peng, Phys. Lett. 87, 38 (1979)

219. G. Montagnoli et al., Phys. Rev. C 97, 044613 (2018)

220. D. Ackermann et al., Nucl. Phys. A 609, 91 (1996)

221. W. Reisdorf et al., Nucl. Phys. A 438, 212 (1985) 
222. W. Reisdorf et al., Nucl. Phys. A 444, 154 (1985)

223. K.T. Lesko et al., Phys. Rev. C 34, 2155 (1986)

224. C.L. Jiang et al., Phys. Rev. C 91, 044602 (2015)

225. K.J. Cook, E.C. Simpson, L.T. Bezzina, M. Dasgupta, D.J. Hinde, K. Banerjee, A.C. Berriman, C. Sengupta, Phys. Rev. Lett. 122, 102501 (2019)

226. A.J. Sierk, Phys. Rev. C 33, 2039 (1986)

227. J. Tōke, G.X. Dai, S. Gralla, A. Gobbi, K.D. Hildenbrand, W.F.J. Müller, A. Olmi, H. Stelzer, B.B. Back, S. Bj, Nucl. Phys. A 440, 327 (1985)

228. W.Q. Shen et al., Phys. Rev. C 36, 115 (1987)

229. B.B. Back, P.B. Fernandez, B.G. Glagola, D. Henderson, S. Kaufman, Phys. Rev. C 53, 1734 (1996)

230. B.B. Back, Phys. Rev. C 31, 185 (2014)

231. P.W. Wen, C.J. Lin, R.G. Nazmitdinov, S.I. Vinisky, O. Chuluunbaatar, A.A. Gusev, A.K. Nasirov, H.M. Jia, A. Gozdz, Phys. Rev. C 103, 054601 (2021)

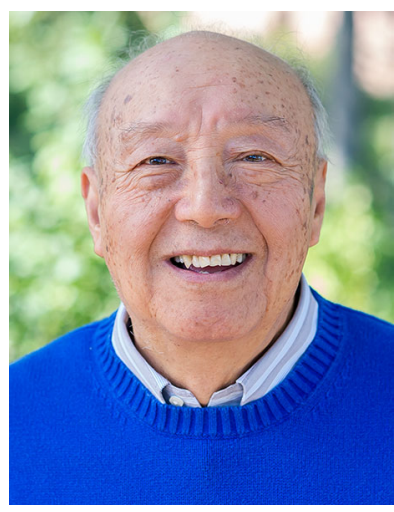

Cheng Li Jiang is an experimental nuclear physicist. He received the Doctoral degree from the China Institute of Atomic Energy, China in 1960 and worked there until his retirement in 1992 as the Director of the Nuclear Physics Division. Since then he became a staff member of Physics Division, Angonne National Laboratory until he retired again in 2005 but continuous to work as an associate of the laboratory. Collaborated with other ANL members, he initiated and developed the study of the heavy-ion fusion hindrance phenomenon at extreme sub-barrier energy around 2002. He was a member of $\mathrm{C} 2$, Nuclear Physics Commission of IUPAP from 1988-1993.

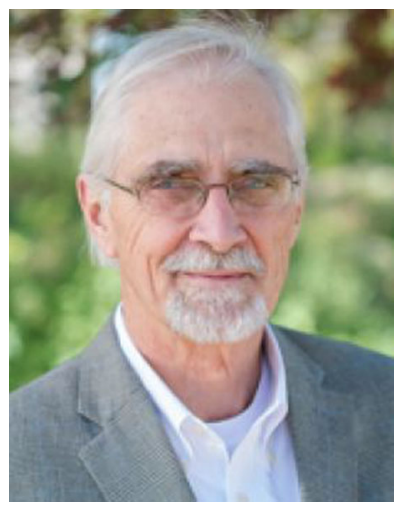

Birger B. Back received the $\mathrm{PhD}$ degree from University of Copenhagen in 1974 in experimental nuclear physics. After short engagements at the Niels Bohr Institute, Gesellschaft fur Schwerionen Forschung, and Los Alamos National Laboratory, he has spent his career at Argonne National Laboratory from 1977 to his retirement in 2020 , but continues his work as a associate of the laboratory. $\mathrm{He}$ has published on a wide range of topics in nuclear physics from fission barrier measurements, heavy-ion fusion-fission reactions, quasifission, heavy-ion fusion, relativistic heavy-ion collisions, and instrument developments. He is a fellow of the American Physical Society.

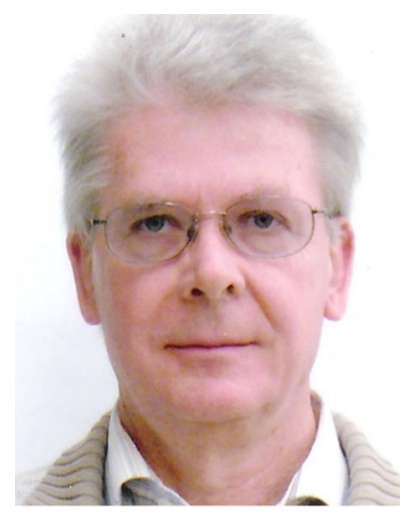

K. Ernst Rehm is an experimental nuclear physicist working in the field of nuclear reactions. He got his $\mathrm{PhD}$ from the Technical University Munich in 1973. He worked as a Scientific Assistant in the TUM Physik Department before joining the Physics Division at Argonne National Laboratory in 1981 . He retired in 2015 as a Senior Scientist. Besides his interest in nuclear reactions, he also works on the development of highefficiency detectors for experiments in nuclear astrophysics using stable and radioactive beams.

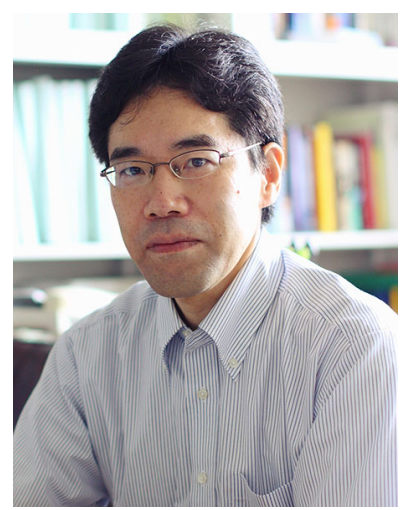

K. Hagino is a theoretical nuclear physicist. He received the doctoral degree from Tohoku University, Japan, in 1998 and worked as a research associate at the Institute for Nuclear Theory, University of Washington, before joining Yukawa Institute for Theoretical Physics, Kyoto University as an assistant professor in 2000. He worked at Tohoku University as an associate professor in 2004-2019. Since 2019, he has been a professor at Kyoto University. He has been working on heavy-ion reactions around the Coulomb barrier, structure and reactions of exotic nuclei, and physics of superheavy elements.

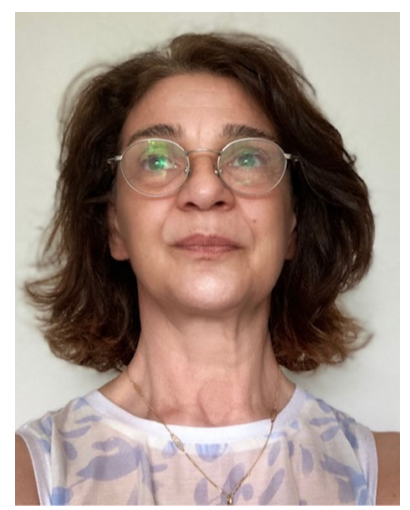

spectrometer PRISMA of LNL.
Giovanna Montagnoli is an experimental nuclear physicist. She received the doctoral degree from the University of Padova in 1987. She was a research fellow at the Technische Universitaet Munich and soon after she obtained an INFN contract at the Laboratori Nazionali di Legnaro. She is presently associate professor at the University of Padova. She has worked in the fields of low-energy heavy ion reactions and of particle detection techniques including the magnetic 


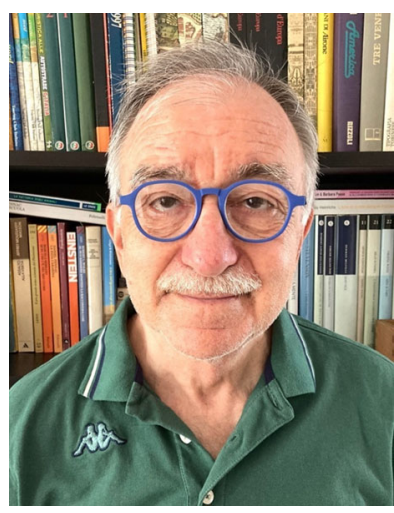

PRISMA magnetic spectrometer.
Alberto M. Stefanini is an experimental nuclear physicist presently senior researcher at the Laboratori Nazionali di Legnaro of INFN. He graduated in Physics at University of Padua (Italy) in 1974. He was a research fellow there and at the KFA of Juelich (Germany), before joining the LNL. He worked on ray spectroscopy of $1 \mathrm{f} 7 / 2$ nuclei, heavy-ion reactions, sub-barrier fusion, and associated detectors and set-ups. He has been PI for the project and installation of the 